Portland State University

PDXScholar

$1-1-1987$

\title{
Determinants of the Spatial Distribution of Peri- Urban to Rural Agriculture in the United States
}

Ibrahim Amin Abdel-Karim

Portland State University

Follow this and additional works at: https://pdxscholar.library.pdx.edu/open_access_etds Let us know how access to this document benefits you.

\section{Recommended Citation}

Abdel-Karim, Ibrahim Amin, "Determinants of the Spatial Distribution of Peri-Urban to Rural Agriculture in the United States" (1987). Dissertations and Theses. Paper 786.

https://doi.org/10.15760/etd.786

This Dissertation is brought to you for free and open access. It has been accepted for inclusion in Dissertations and Theses by an authorized administrator of PDXScholar. Please contact us if we can make this document more accessible: pdxscholar@pdx.edu. 


\section{DETERMINANTS OF THE SPATIAL DISTRIBUTION OF PERI-URBAN TO RURAL AGRICULTURE IN THE UNITED STATES}

by

IBRAHIM AMIN ABDEL-KARIM

A dissertation submitted in partial fulfillment of the requirements for the degree of

DOCTOR OF PHILOSOPHY

in

URBAN STUDIES

Portland State University

1987 


\section{TO THE OFFICE OF GRADUATE STUDIES AND RESEARCH}

The members of the Committee approve the dissertation of Ibrahim Amin AbdelKarim presented December, 1987.

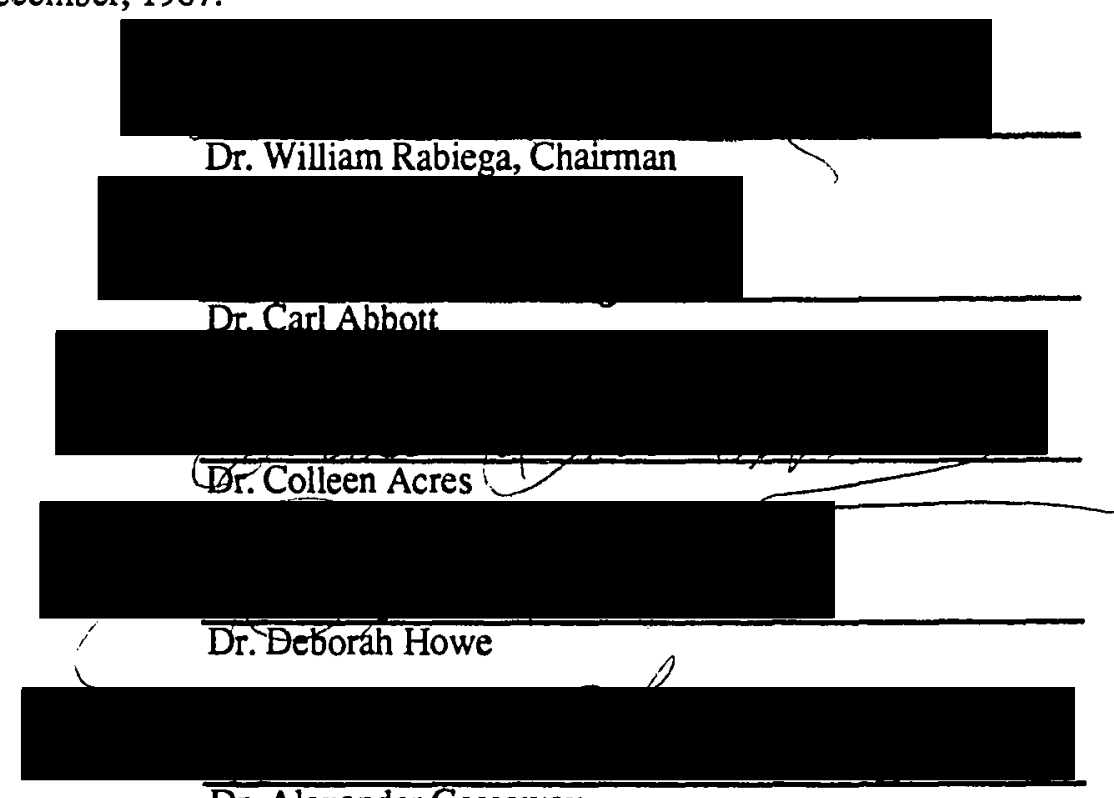

Dr. Alexander Gassaway

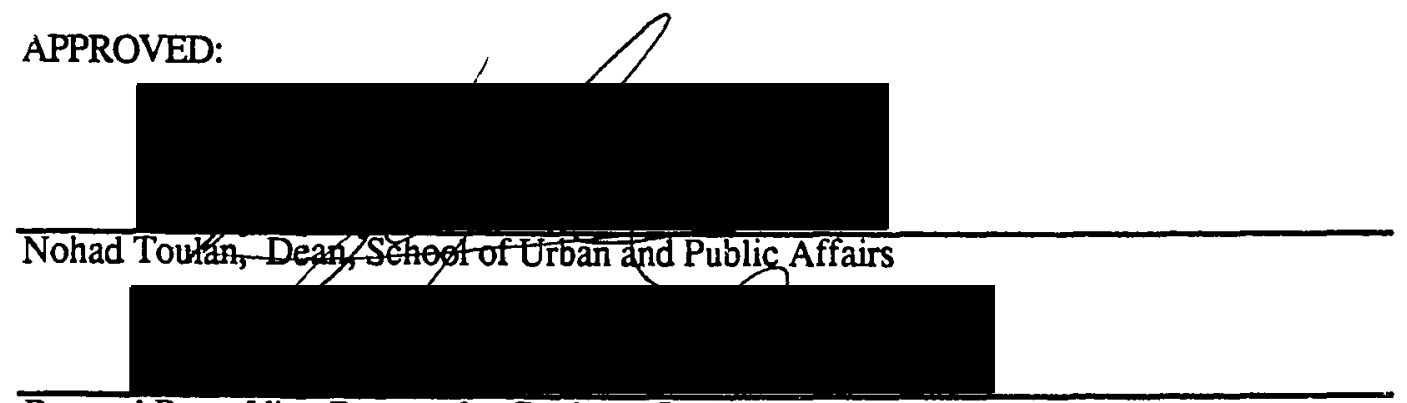

Bernard Ross, Vice-Provost for Graduate Studies 
AN ABSTRACT OF THE DISSERTATION OF Ibrahim Amin Abdel-Karim for the Doctor of Philosophy in Urban Studies presented December, 1987.

Title: Determinants of the Spatial Distribution of Peri-Urban to Rural Agriculture in the United States.

\section{APPROVED BY MEMBERS OF THE DISSERTATION COMMITTEE:}

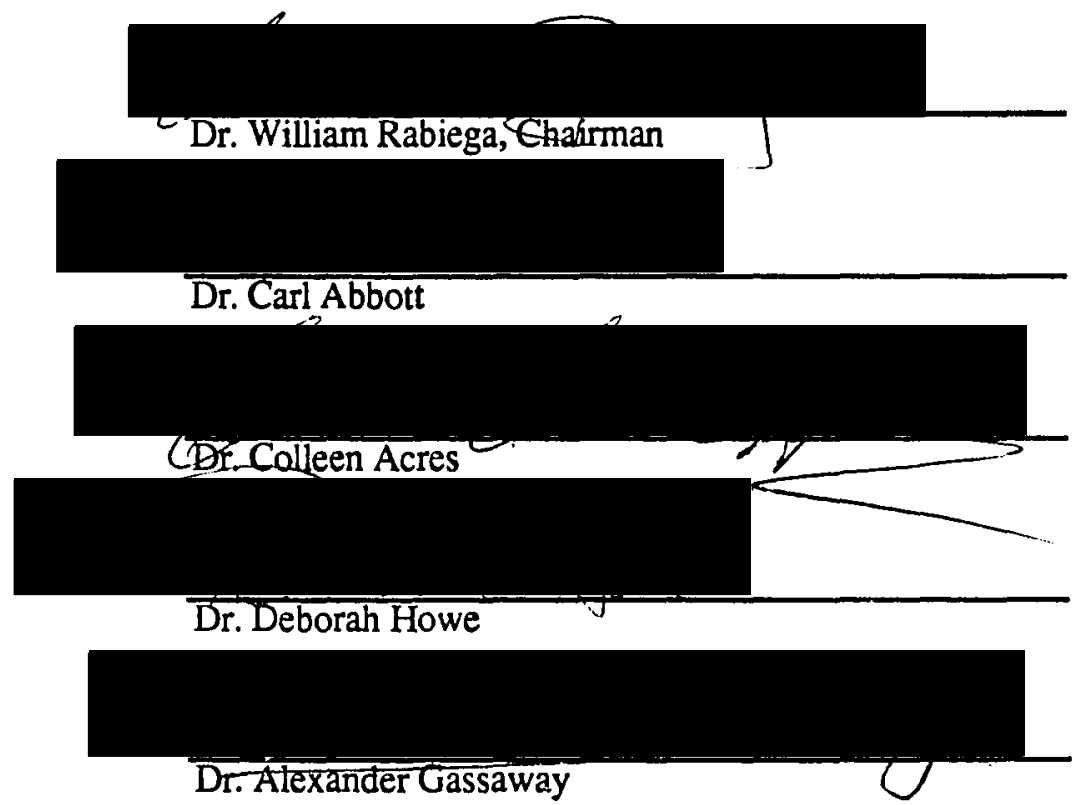

Two issues are focal to the subject of the spatial distribution of crops in peri-urban zones. The first deals with developments in the fields of transportation, other technology, urbanization, and other factors which are not only prevalent in developed world economies, but which also are thought to force the cultivation of freshly consumed agricultural commodities away from the immediate vicinity of the market center. The second issue pertains to indirectly consumed crops, which are thought to shun proximity to the market center, where land rents per unit area are characteristically high, even when conditions for 
productions are ideal. Traditional models have shown a zonal pattern of crop distribution in peri-urban areas.

The present study sets forth two hypotheses, one pertaining to the spatial distribution of freshly consumed crops, and the other pertaining to the spatial distribution of indirectly consumed crops.

It was hypothesized in the present study that freshly consumed crops will continue to be cultivated in the near vicinity of the market center due to characteristics of the crops themselves and to features of the urban market. It was further hypothesized that indirectly consumed crops will continue to be cultivated in the near vicinity of the market center by virtue of greater intensity of production that may be obtained through the use of the environment of designated places. In the case of both crops, the cited factors, as well as others, offset the disadvantages of higher land rent per unit area common to areas close to the market center. These offsetting factors permit agriculture to compete successfully for land in the peri-urban zone.

To test these hypotheses, variables were selected to measure the influence that urbanization, transportation, other technologies, the market, the environment, and land use regulations have on agricultural siting pattems in the peri-urban zones of the "Wheat Region" of the central United States. These variables were expressed as equations and were subjected to multiple linear regression (MLR) analysis.

The present findings tended to support the research hypotheses. On the basis of these findings, the present research offers a revised model of agricultural cropping patterns, one that reflects the sectoral, rather than the zonal, pattern of crop distribution in peri-urban zones. In the revised model, the mixing of different crops at various locations around the market is feasible, and low-priced grains may compete successfully for high-rent locations in the near vicinity of the urban market. The findings also show that the production of perishable crops in the immediate vicinity of the urban market is here to stay, largely due to 
access to varied means of transportation as well as characteristics of the crops themselves.

Furthermore, the findings show that environmental conditions influence the locating of grain production, although economic considerations were seen to supersede them, particularly at high-rent sites. 


\section{ACKNOWLEDGEMENT}

This work could not have been finished in its present form without the concerted effort of people too numerous to name. To them I pay tribute. I am obliged to express my gratitude as well to the committee that supervised the progress of this research throughout its course. I also wish to recognize the efforts of Dr. William Rabiega, Dr. Alexander Gassaway, and Mr. Nick Gattuccio. 


\section{DEDICATION}

This work is dedicated to my parents, my wife, Jalileh, and to our child, Salam, as a gesture of love and gratitude.

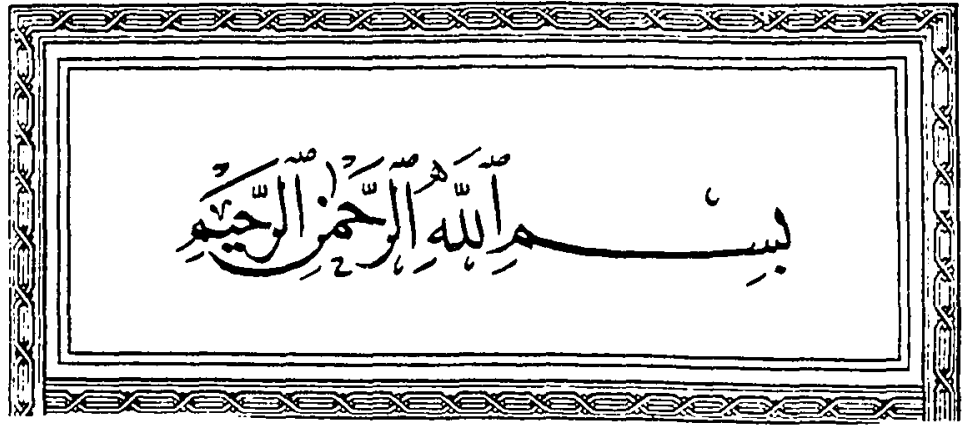




\section{TABLE OF: CONTENTS}

CHAPTER

PAGE

I. INTRODUCTION ........................................................

Problem and Hypothesis...............................................2

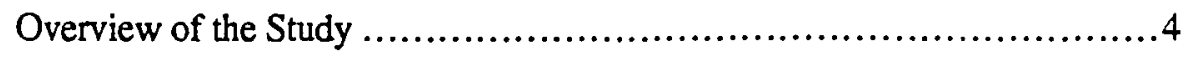

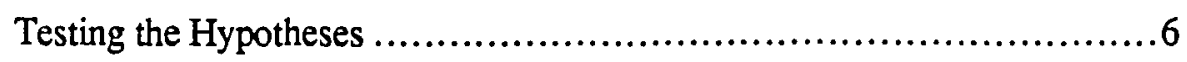

II. A REVIEW OF THE THEORETICAL LITERATUE ON

PERI-URBAN AGRICULTURE $\ldots \ldots \ldots \ldots \ldots \ldots \ldots \ldots \ldots \ldots \ldots \ldots \ldots . \ldots$

Introduction .........................................................

The Theory of Von Thunen.................................................9

The Land Rent Factor in the Isolated State................................... 12

The Impact of the Land Rent Gradient on the Location of

Crops Around the Urban Market................................. 15

The Intensity Theory of Production in the Isolated State..................... 18

Applicability of the Isolated State to Present-Day

Peri-Urban Agriculture ......................................... 22

Points Common to the Three Theorists on Peri-Urban

Agriculture ................................................... 31

Other Views on the Isolated State.................................... 32

III. THE IMPACT OF TECHNOLOGICAL INNOVATION ON

THE LOCATION OF INDIVIDUAL CROPS IN THE

PERI-URBAN FRINGE ........................................... 37

Introdustion ..................................................... 37

Changes in the Location of Perishables Production Relative to the Market Center ..................................... 38

The Two-Location Initiative of Perishables Production................... 41

The Roots of the Dichotomy in the Location of Perishables

Production ......................................................... 43

The Fundamental Question ..................................... 47 
The Cultivation of Perishables in the Near Vicinity of the Urban Market 48

Shifts in the Location of Grain Production Relative to the Urban Market.................................................... 52

IV. RESEARCH DESIGN AND CONSTRUCTION OF THE MODEL ............ 57

Hypothesis Number One: The Location of Perishable Crops Production in the Peri-Urban Zone ................................. 58

Hypothesis Number Two: The Location of Grain Production in the Peri-Urban Zone .............................................. 64

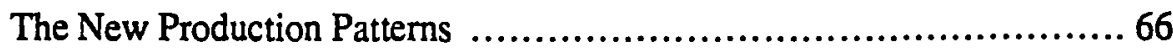

The Empirical Analysis.................................................... 70

The Model .............................................................. 73

The Units of Analysis .............................................. 75

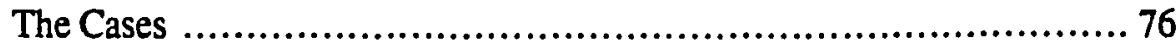

The Variables ....................................................... 76

Equations for Testing the Hypotheses ................................ 90

Impacts of the Market Factor on the Cultivation of the Wheat Crop .............................................. 91

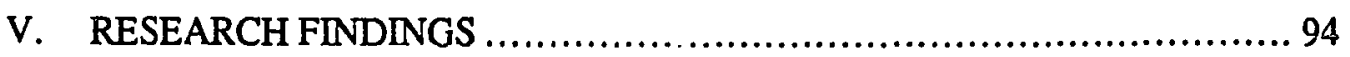

Introduction........................................................ 94

Preliminary Analysis of the Data....................................... 95

Guidelines for Data Interpretation......................................... 99

Analysis of the Regression Output.........................................100

Phases of Data Analysis....................................................100

Empirical Testing of the Cultivation of the Wheat Crop......................105

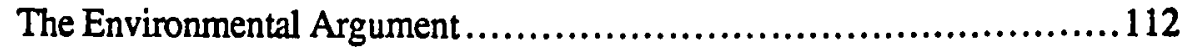

Other Variables in the Equation ........................................115

Empirical Testing of the Cultivation of the Vegetable Crop...................116 
Empirical Testing of the Production of Greennouse Culture and Nursery Production in Peri-Urban Zones......................127

VI. SUMMARY ANALYSIS AND CONCLUSIONS ............................137

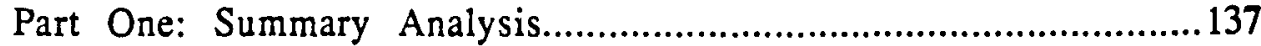

The Accessibility Variable ............................................137

Other Predictors in the Equation ..........................................139

The Impact of the Marketplace .......................................... 142

The Impact of Urbanization............................................... 144

The Impact of the Environmental Factors..................................145

Part Two: Conclusions....................................................... 148

Characteristics Unique to the Present Research .......................... 149

Contemporary Determinants of Peri-Urban Cropping Patterns ............150

A Sector Theory of Peri-Urban Cropping Patterns :...................... 152

Transportation Technology and Peri-Urban Agriculture .................... 153

Technology Variability and Crop Location ...................................155

The Intensity of Crop Production ...................................158

The Role of the Environment in the Location of Peri-Urban

Agriculture ...................................................... 161

The Role of Urbanization on the Location of Peri-Urban

Agriculture ................................................. 161

Land Use Regulations and the Location of Peri-Urban

Agriculture ................................................... 162

The Research Findings and Present Theory .............................163

Technology and the Location of Peri-Urban Agriculture....................... 163

Part Three: Implications of the Present Research ............................... 165

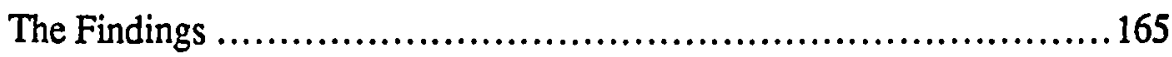


Discussion of the Study Design and Recommendations

for Future Research........................................ 167

The Isolated State in a Sectoral View of Agricultural Production ............168

BIBLIOGRAPHY ......................................................... 170 


\section{LIST OF TABLES}

TABLE

PAGE

I Quantities and Pricing of Wheat Produced in the United States

for Selected Years

II Distribution of Agricultural Production in Peri-Urban Zones,

by Crop and County Tier .............................................. 96

III Distribution of Agricultural Production in Peri-Urban Zones, by Crop

and Location, Expressed as Percentage of Absolute Values

IV Distribution of Agricultural Production in Peri-Urban Zones, by Crop

and Location, Expressed as Percentage of Adjusted Total

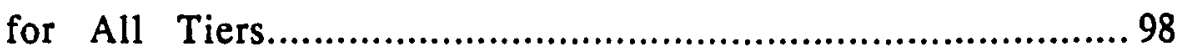

V Regression Output for the Acreage of Harvested Wheat

at Three Locations in the Peri-Urban Zone

VI Beta Values of Regression Output for Acreage of Wheat

Harvested at Three Locations in the Peri-Urban Zone 109

VII Beta Values for the Variables PRECIPIT and GROWTH for the

Criterion Variable Wheat Production at Four

Environmental Subregions.

VIII Regression Output for the Acreage of Harvested Vegetables

at Three Locations in the Peri-Urban Zone

IX Beta Values of Regression Output for Acreage of Vegetables

Harvested at Three Locations in the Peri-Urban Zone.

X Beta Values for Regression Output for Acreage of Vegetables

Harvested at Three Locations in the Peri-Urban Zone

With the Elimination of the Variable INTERACT. 
Xi Regression Output for Greenhouse Culture and Nursery

Production at Three Locations in the Peri-Urban Zone.........................129

XII Beta Values of Regression Output for Greenhouse Culture and

Nursery Production at Three Locations in the Peri-Urban Zone ............131

XIII Beta Values for the Variables PRECIPIT and GROWTH for the

Criterion Variable Greenhouse Culture and Nursery Production

in Four Environmental Subregions .................................135 


\section{LIST OF FIGURES}

FIGURE

PAGE

1. Agricultural Cropping Patterns as Depicted in the Isolated State......................9

2. The Economic Rent Gradient for Agricultural Sites in the Near

Vicinity of SMSA Market Centers ................................... 15

3. Agricultural Cropping Patterns as Resulting From Von Thunen's

Isolated State, as Modified by Schlebecker (1973) ...................... 25

4. Land Utilization in the Peri-Urban and Extended Zone as

Predicted by Sinclair (1967) ......................................... 29

5. Agricultural Cropping Patterns in the Peri-Urban Fringe of

Metropolitan Urban Centers (SMSAs) ............................. 67

6. Agricultural Cropping Patterns in the Peri-Urban Fringe of Nonmetropolitan

Urban Centers (with populations less than 50,000 ) ..................... 68

7. The Spatial Distribution of the Five Primary Varieties of

Wheat in the United States......................................... 72

8. Urban, Second-, and Third-Tier Counties of States in the

U.S. Wheat Region................................................... 75b

9. Summary of Variables and Data Sources .................................... 89

10. Graphic Representation of Crop Distribution Data as Presented

in Tables III and IV.................................................. 99 


\section{CHAPTER I}

\section{INTRODUCTION}

Much of the world's agricultural land use is located on the fringe of urban centers. These areas are known as peri-urban agricultural areas, or zones (Organization of Economic Cooperation [OECD], Vol. I, 1979). The purpose of the present study is to investigate spatial patterns of crop location in these peri-urban zones and to assess the factors responsible for crop-selection and location decisions. This study will focus on conditions in the United States.

Peri-urban zones are areas that lie between urban and rural settings and which experience impacts from both directions. Due to this placement, these zones are often described as "grey areas," neither wholly urban nor particularly rural in the traditional sense. Rather, they may be described as at most partly urbanized rural areas. This ambiguity in defining the character of peri-urban zones also creates uncertainties when attempting to draw specific boundaries for the zones, as well as when trying to specify factors responsible for agricultural land uses occurring in the zones. According to the OECD (1979), peri-urban areas can be identified on the basis of the kinds of urban activities found encroaching on the zone, as well as by the kinds of crops that are cultivated in the area. The OECD finds it difficult to determine the depth of the zone in any given area, however, since geographical proportions of land uses vary considerably with the degree of spread of the kinds of urban activities that are characteristically used to define a peri-urban zone.

This inherent ambiguity in locating and defining the peri-urban zone is reflected in 
the literature, which is sketchy and lacks consistency. There is no definitive variable or set of variables that portray agricultural cropping patterns in these areas. Views vary considerably as to the crops that tend to be grown in peri-urban zones as well as on the factors (rural, technological, environmental, etc.) that most prominently influence their selection and intensity of production.

Some researchers ascribe the spatial distribution of agriculture in peri-urban zones to the production site's linear distance to a market in the immediate urban area, combined with the characteristics of the commodity transferred (Von Thunen, 1826) in his Isolated State hypothesis; others associate these patterns with the impact of urbanization on periurban agriculture (Sinclair, 1967). A third group considers patterns of consumption and transportation technology to be the principal factors influencing the selection and location of crops around a city (Schlebecker, 1973). Yet a fourth group holds that the natural environment is the predominant influence on the selection of crops cultivated in the near vicinity of population centers, i.e., peri-urban zones (Taylor, 1942). Finally, others, such as Bryant (1983), see land use patterns in peri-urban zones resulting from a comprehensive process involving many human and environmental factors.

\section{PROBLEM AND HYPOTHESES}

This study posits that perishable crops are cultivated in peri-urban zones of metropolitan, as well as many nonmetropolitan, urban centers, in a pattern is due more significantly to urbanization and the characteristics of the perishable crops themselves than to the mere fact of convenient proximity to the metropolitan market, as is suggested in some theoretical literature on the subject. Furthermore, it is hypothesized that grains, whether for human consumption or for livestock feed, are so strongly influenced by environmental factors that under the right environmental conditions grain cultivation will extend into peri- 
urban zones, regardiess of the higher land rents associated with these areas and despite the fact that the nearby urban centers do not constitute direct markets for the grain produced.

The above two contentions define the scope of the present research. Several factors reinforce the significance of work in this area. First, a majority of the literature on periurban agriculture suggests that developments in the fields of transportation, food preservation, and agricultural technology make it highly unlikely that one may satisfactorily determine cropping patterns for perishables, particularly in developed countries like the United States, by means of applying Von Thunen's Isolated State (1826). It is maintained instead that the simple fact of perishability has ceased to be a significantly limiting factor in the locating of perishables production, particularly at sites more distant from urban centers that possess better growing conditions and retain the advantages of competitive transportation links with the urban market.

Second, it is held that due to grain production's relatively low financial return per acre, and the characteristically high acreage needs for successful grain cultivation, grain production in the near vicinity of urban centers is severely discouraged. However, some recent research in the field of peri-urban agriculture dispute this assumption (Heid, 1979; İkerd, 1985; Schneider, 1986). Sinclair's work (1967) lays an economic foundation for grain cultivation in the peri-urban areas of growing cities.

Third, research on this topic has tended to approach peri-urban agriculture as a "lump-sum" process, wherein all crops were discussed collectively, with sut considering the production, marketing, and transportability characteristics of each crop. In the present study each will be treated individually. Furthermore, this study will compare and contrast its findings with theoretical research on peri-urban agriculture, the majority of which is grounded in the work of Von Thunen (1826), which is centered in his theory of the Isolated State. This theoretical approach proposes the absolute significance of agricultural production's linear distance to the nearest market as the criterion regulating the spatial 
distribution of crops around that uiban market center. By virtue of technology existing at that time (1826)--transportation, refrigeration, etc.--Von Thunen introduces six concentric zones of production beginning at the innermost ring with crops that are most prone to spoilage, and concluding in the outermost ring with stock farming--a product far less subject to spoilage and that literally walks itself to market.

Fourth, the present research will address questions about urbanization and urban encroachment and its impact at different levels on the location of various crops in the periurban zone, and will compare particularly impacts in the metropolitan versus urban nonmetropolitan centers.

\section{OVERVIEW OF THE STUDY}

This study will assess cropping patterns of peri-urban agriculture around the urban markets in the United States, and will assess which factors are most responsible for these pattems of production. The analysis will focus on the effects of urbanization and new technologies on the location of production in these peri-urban areas. It will further address the extent to which new technologies have succeeded in dispersing agricultural production away from the market center, irrespective of the perishability of the crop itself or its means of consumption (i.e., directly or indirectly).

The present study will also investigate the impact of characteristics of the crops themselves on their spatial distribution. This may be significant considering that not all agricultural production is consumed directly by humans. Rather, some crops (for example, many grains) require processing before being marketed to humans, and thus are consumed indirectly. An added factor is that a large proportion of these grains are intended for foreign markets. This raises the question of what factors would induce a producer, who is motivated primarily by profit, to risk cultivating an export grain crop in peri-urban sites, where land is characteristically expensive per unit area. 
Also to be considered is the question of "new" locations, if any, for crops consumed directly by humans and that are marketed in an unprocessed, or "fresh," condition. This is focal particularly when these crops provide the producer with the option of cultivating "outer" locations, which generally have not only lower land rent per unit area but also exhibit environmental conditions that are more amenable to the crops under cultivation. At the same time, these outer locations still provide their producers with transportation networks that allow access to proximate markets at rates competitive with closer-in locations once the latter's higher land rent is taken into account. In light of this, one would ask whether it remains profitable to produce freshly consumed crops in the immediate vicinity of the market (that is, in the peri-urban zone), even in cases where the environmental conditions for the cultivation of these crops are less than ideal?

Such an inquiry includes, among other things, a discussion of the characteristics of the population center as well as characteristics of the crop under cultivation. This would include prevailing environmental conditions that may override the impacts of agricultural and transportation technology as well as land rent per unit area in decisions on crop siting in peri-urban zones. This matter is treated in a way that reveals different production and distribution strategies that result in differing cropping patterns in peri-urban areas, irrespective of the unifying impacts on both production and distribution of new technology and environmental influences.

The present research will attempt to answer the following questions: Are the crops produced in peri-urban zones determined by the geographical location alone? If not, is their location a result of (a) environmental factors? (b) advances in transportation, food-preservation, and agriculture technologies? or (c) characteristics of the crops themselves--that is, by their marketability locally--and the characteristics of the proximate urban center? These questions define the parameters of the present research.

One of the primary research questions which this study addresses concerns crops 
that fuifill the foliowing criteria: (a) they are consumed fresh; (b) they may be produced in outer locations, under more convenient environmental conditions than are available in periurban zones, and where their producers would pay less rent per unit area; and (c) they are marketable at competitive rates due to available transportation and agricultural technologies. This study asks, If given these circumstances, would these crops still be cultivated in periurban areas where land rent is high and where environmental conditions may not be ideal $\mathrm{fo}_{\mathrm{L}}$ ineir production?

A related question concems a second kind of agricultural product--that is, crops that are not consumed directly (that is, "fresh") by humans, therefore removing any immediate incentive for production in the near proximity of an urban market. The concern here is with grain crops, and the central question is this: Would indirectly consumed crops tend to avoid the near proximity of the urban market, where land rent is generally high, and seek outer locations, even if environmental and other conditions at the close-in sites are ideal for high production of these crops?

The above two sets of questions are two ways of addressing the same set of landuse conditions, but from different directions. Answering these questions, which address the forces that come to bear on, and the influences that largely determine agricultural land use patterns in peri-urban zones, is the central intention of the present study.

\section{TESTING THE HYPOTHESES}

The research hypotheses will be tested empirically. A multiple linear regression (MIR) will be performed, as it is a proven technique for analyzing such additive and straightforward correlations. The crops selected for this analysis are wheat, vegetables, greenhouse culture, and nursery products.

Data for this study were collected from the "wheat region" of the United States (see Figure 7). This region was selected because it provides a broad and representative 
sampling of the demographic, agricuitural, and other developmental and urbanizationrelated conditions which the present research addresses. The variabies selected for testing the hypotheses will cover the physical environment, transportation, new technologies, urbanization, as well as characteristics of the marketplace and of the crops themselves.

The study will rely on 1980 data; this year's data were selected because their statistics show them to be "typical" in a series extending between the mid-1970s and early 1980s, an era that witnessed a peak in U.S. agricultural export, particularly for grain crops, as well as peak levels of federal price supports and prices for goods sold. 


\section{CHAPTER II}

\section{A REVIEW OF THE THEORETICAL LITERATURE ON PERI-URBAN AGRICULTURE}

\section{INTRODUCTION}

The theoretical literature on peri-urban agriculture begins with Von Thunen's Isolated State (1826), which is considered the first and still the only comprehensive work in this field which rests on both theoretical and empirical foundations (Hall, 1966; Jones et al., 1978). Von Thunen addresses both the cropping and intensity patterns of agriculture in this zone.

Subsequent theoretical literature on the subject includes the work of Sinclair (1967) and of Schlebecker (1973). Both writers reflect on the views of, and present reactions to, Von Thunen's Isolated State. Their works and those of others on the subject are extremely diverse; however, all relevent views will be reviewed. All of the theoretical views of peri-urban agriculture are formulated on the basis of studies conducted primarily within economically developed regions of the world.

For the sake of organization, this study will treat peri-urban agriculture in two major divisions: crop production patterns (cropping patterns) and production intensity Fatteris. The study will favor the former, but intensity patterns will be introduced when appropriate, since the level of intensity of production is a major determinant of yields and revenues per unit area, and consequently influences the location of production relative to market centers. 


\section{THE THEORY OF VON THUNEN}

Most prominent in the literature on the spatial distribution of agriculture in peri-urban zones and the factors responsible for it are the views of Johann Heinrich Von Thunen presented in The Isolated State (1826) (see Figure 1).

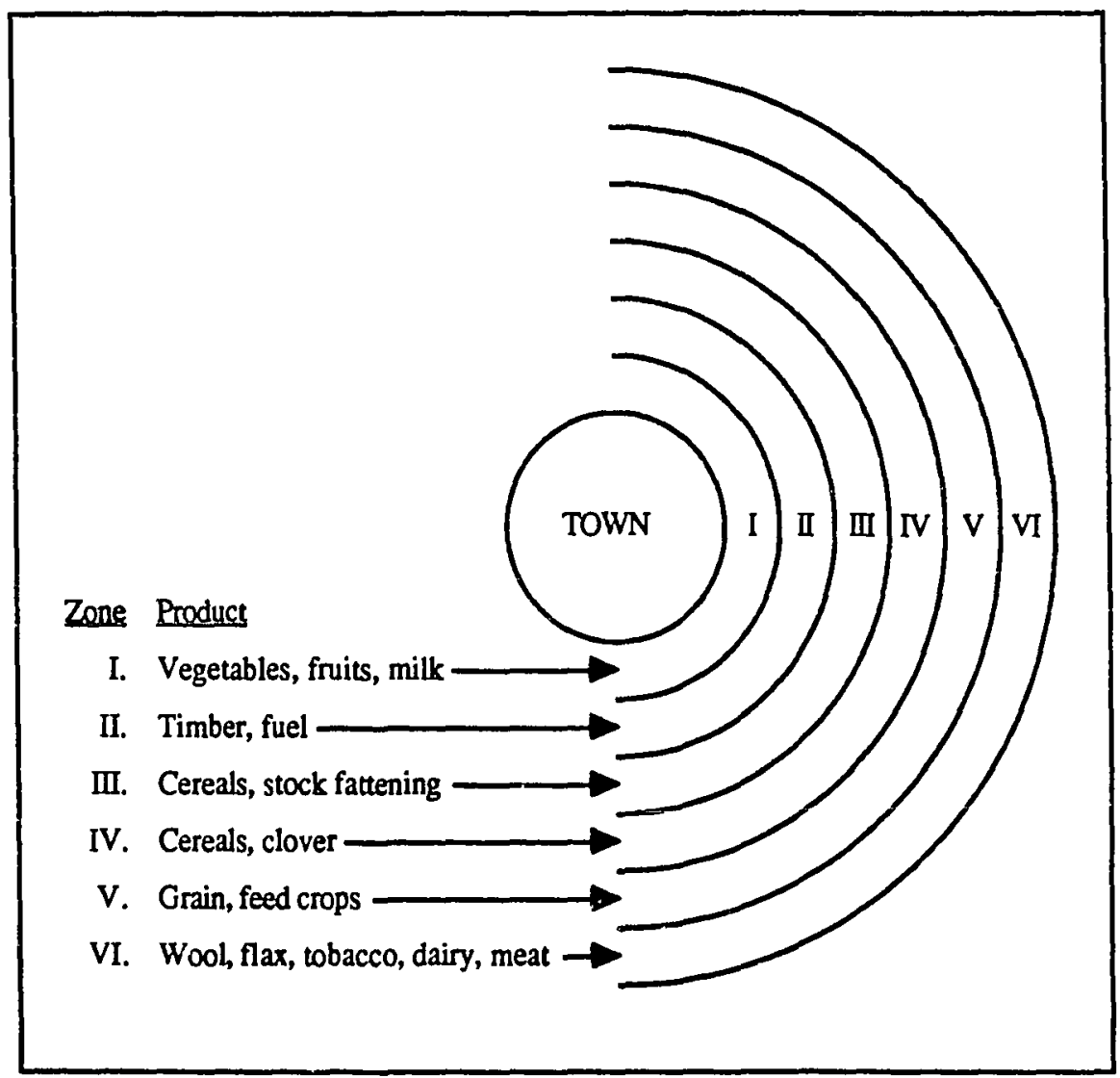

SOURCE: Johnson (1970).

Figure 1. Agricultural cropping patterns as depicted in the Isolated State.

Integral to Von Thunen's view was the effect of the linear distance between the production site and the urban market, as well as the characteristics of the commodity transferred. These factors affected a perishable crop's feasibility for transport and the costs of movement. Grotewald (1959) ascribes this prominence Von Thunen gives to 
transportation factors to characteristics of transportation available at that time (the early nineteenth century) and at the place of Von Thunen's residence, Mecklenburg, a region in the very north of present-day East Germany. When Von Thunen first made it his home, Mecklenburg had neither major navigable rivers nor improved roads. Not until after his death in the mid-1860s did the railway penetrate even to Teterow, the town nearest his own town of Tellow, and only by the 1880 s had a branch of the railroad approached Tellow (Hall, 1966:xvi). This crude state of transportation in the area seems to have influenced Von Thunen's choice of the horse-drawn cart as the sole means of transportation within his hypothetical "idealistic" state. Moreover, these same circumstances appear to be responsible for his identifying his proportion-to-distance charges which he assigned to commodities transferred within that state.

Other of Von Thunen's theoretical assumptions include the existence of only one urban center for marketing the "state's" agricultural production. That urban center would be located in the middle of the state, amid a featureless plain of uniformly fertile soil. Farmers and agricultural entrepreneurs were assumed to be evenly distributed around the market center, uniformly cultivating the land and marketing their crops. Transactions were assumed to occur in a free market with free entry; neither extra profits nor changes in the going market price of commodities sold in that market exceeded the capacity of any one producer or a limited group of producers to deal with. Finally, the state was assumed to be fully isolated from all outside influences (Peet, 1969; Schlebecker, 1973; Jones et al, 1978).

By design, it is this very exclusivity in structuring the Isolated State that caused the intended elimination of the impacts of any and all factors other than transportation costs and characteristics of the commodity transported in constructing a model for the forces influencing the cultivation of land in peri-urban zones (Grotewald, 1959). The merits of 
such an approach are obvious: It provides a constant environment of analysis, asceteris paribus, freezing all factors in the model except the variables under study. According to Datoo (1978), a study with this rigidity of control helps explain the determinants of the process in agricultural change. Many of the variables that interact to produce the economic landscape of a region contribute toward both cause and effect. Thus, it becomes worthwhile to isolaie the pursued variables from all other economic, social, cultural, and even psychological factors in order to ensure that the variables intended for analysis are those that are in actuality measured (Lloyd, 1977). Von Thunen is seeking a complete manipulation of the variables in question. It is doing just this, Lloyd suggests, that requires the researcher to know most precisely which variables are involved in the process, and also what specific assumptions need to be made about their action in the model. Since this last point amounts to identifying the rules of the game, so to speak, Von Thunen's success at identifying the variables and their activity in the model constitutes his most important accomplishment, as he himself saw it.

Tarrant (1974) explains certain of Von Thunen's theoretical assumptions as follows:

In Von Thunen's Isolated State, demand is fixed and predictable, and so, therefore, is supply. In the perfect world of such a model, supply can be matched to demand because a uniform and stable physical environment and the operation of farmers in a state of perfect knowledge are assumed. This type of model has its uses, particularly in illustrating how certain variables operate in isolation from the effects of others. (pp. 200-201)

Even by today's standards such a controlled model is significant, since it provides a laboratory-type environment for analysis that is essential to both the physical and the social sciences. 


\title{
THE LAND RENT FACTOR IN
}

\section{THE ISOLATED STATE}

\begin{abstract}
A fundamental premise of the Isolated State is the rise in the value of land rent per unit area in the direction of the market center. This factor is tied not only to actual land values, but also to transportation costs, since Von Thunen establishes the latter as directly proportional to distance to the market center. These criteria are not only largely responsible for cropping pattems in the Isolated State, but intensity patterns are also closely tied to the land-rent gradient that Von Thunen presents. In fact, the rent criterion is the major determinant for almost all profit-oriented activities competing for sites in the Isolated State.

Holding every force or variable other than transportation cost-over-distance constant, as this model proposes, renders agricultural sites that are closer to the market center more economic advantages than sites that are more distant. This is a function of the cost-economics of transportation, which in the Isolated State are proportionai to the distance from farm to market. The advantage for marketing agricultural output, then, goes to sites closer to the urban market. Thus, the closer one approaches the urban center, the greater the attractiveness of agricultural production sites in terms of transport costs, and land rent increases approximately proportional to the degree of attractiveness. Hence, competition for the more advantageous sites closer to the market center increases their costh offsetting proximity to the market to a variable degree. The interaction of these two factors is critical to making decisions about crops to be produced and their location relative to the market center, since the interaction of these two factors decides to a large degree the value of "economic rent" at each site.

The term "economic rent" here is used in the classical sense: "To describe payments to any factor that is in completely inelastic supply" (Gill, 1973:491-493). This classical definition is the same as that provided by Von Thunen for his term "land rent," which Hall
\end{abstract}


(1966:19) restates as follows: "That portion of the farm revenue that is left after deduction of the interest on the value of the buildings, timber, fences, and all other valuable objects separable from the land, that portion which pertains to the land itself, is land rent."

Schlebecker (1973) views this rise in the value of land rent per unit area in the direction of the market center as a universal phenomenon, a view that is shared by Alonso (1960) and many others. However, views vary considerably on determining precisely which factors are most responsible for the rise. Krzymowski (1926), for example, ascribes it to the fact that commodities with similar characteristics sell for the same price per unit product in the market, regardless of where they are produced. This attracts more producers to locations in the near vicinity of the market than to sites farther out. Alonso (1960) sees land in the near vicinity of the market valued at the same time for both its real estate value and as an agricultural location. Cromely (1982) sees the locational advantages of sites in the market's vicinity exceeding the transportation costs that farmers on more distant sites pay for marketing their produce. This again suggests that transportation alone is not the major determinant of agricultural production sites.

Other researchers ascribe this rise in land value in the direction of the market center to the monopoly privileges accruing to sites closer to the market by way of access to services originating in the nearby urban center, to which more distart sites have less access. Jones (1978) reports a lower failure rate for agricultural businesses located nearer to urban centers. He explains this as resulting from increased costs that farms farther from the urban market must pay to transport and market their produce, which leaves their net retums more vulnerable to market fluctuations.

According to Von Thunen's calculations, net returns, and hence land rents, are determined neither by a commodity's sale price nor the net retum per load of produce. If such were the case, crops with a higher market selling price per unit product would be 
drawn closer to the market center, where land rent is higher, and lower priced commodities would be forced out toward more distant sites with lower rent per unit area. Calculating land rent according to Von Thunen involves the figure for yield per unit area. This introduces into the formula a host of factors that influence the determination of rents at sites in the near vicinity of the urban market. These additional factors include transportability of the commodity, bulkiness of the product, and the product's market selling price. This leads us to a formula which Von Thunen used to calculate land rent:

$$
\begin{aligned}
& \mathrm{L}=\mathrm{P}-\mathrm{A}-\mathrm{R}, \text { where } \\
& \begin{array}{l}
\mathrm{L}=\text { rent } \\
\mathrm{P}=\text { total production } \\
\mathrm{A}=\text { wages } \\
\mathbf{R}=\text { capital utilization }
\end{array}
\end{aligned}
$$

Subsequent writers on the subject have used this basic formula, but with the slight modification. Whereas for Von Thunen transportation costs were implicit, as the above formula shows, in later versions these costs are clearly stated. A good example of this may be seen in the following formula prepared by Dunn (1954):

$$
\begin{aligned}
& R=E(P-A)-E F K, \text { where } \\
& \mathbf{R}=\text { rent per unit land } \\
& \mathrm{K}=\text { distance } \\
& \mathbf{E}=\text { yield per unit land } \\
& \mathbf{P}=\text { market price per unit product } \\
& A=\text { production cost per unit product } \\
& F=\text { transportation cost per unit distance per commodity }
\end{aligned}
$$


The formula above describes rent as a function more of distance than of output, which Figure 2 (below) describes graphically. It should be noted in Figure 2 that the marginal bid rent curve is substituted for the economic bid rent curve, and that the value of land rent is substituted for output. Thus, land rent is substituting for distance. Remember here that transportation costs are established proportional to the distance traveled.

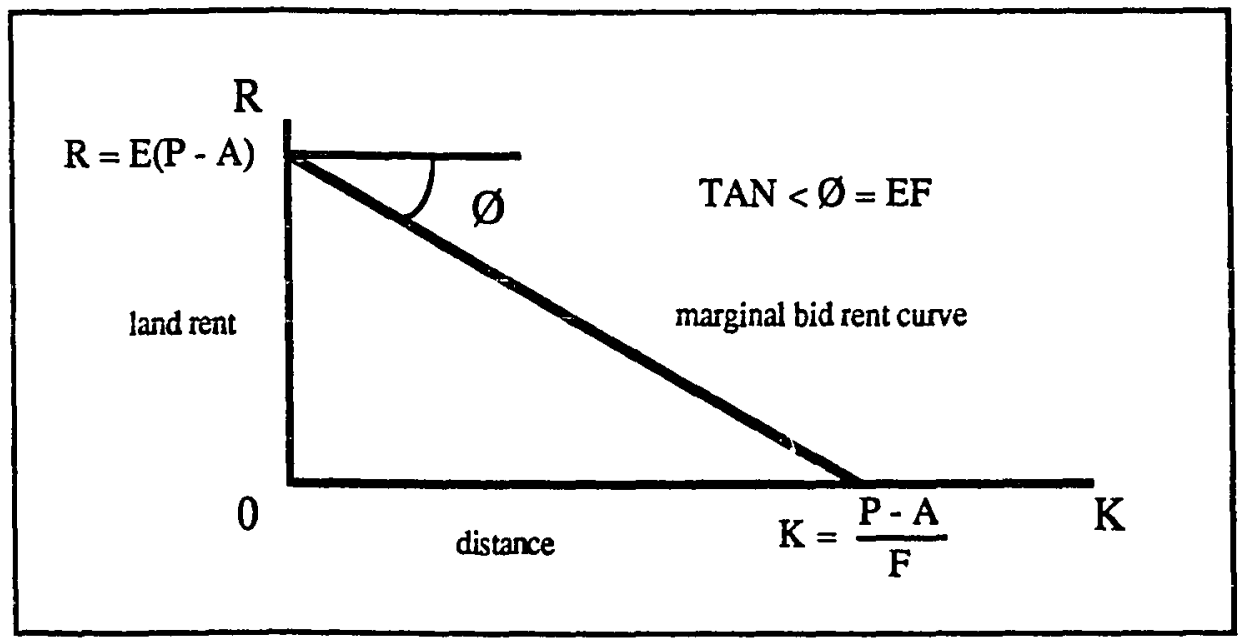

SOURCE: Dunn (1954:7)

Figure 2. The economic rent gradient for farm sites in the near vicinity of market centers.

\section{IMPACT OF THE LAND RENT GRADIENT ON THE LOCATION OF CROPS AROUND THE URBAN MARKET}

Dunn (1954) acknowledges the lead of Von Thunen in the issue of land rent. He and other writers realize that the controlling factor in determining land use is land rent. $\mathrm{He}$ explains the effect of land rent on land use in the following terms: "That form of land use which provides the greatest rent will make the highest bid for the land and hence displace all others" (p. 233). However, Dunn also values distance per se as a significant determinant of crop location, saying: "If the previous theorists in the field of agricultural 
location have been guilty of a common weakness, it is their failure to develop an explicit distance function" (p. 233).

In Dunn's formula, shown above, rent $(R)$ is the dependent variable, and distance (K) the independent variable, with $\mathrm{P}, \mathrm{A}$ and $\mathrm{E}$ acting as constants, or parameters. Dunn distinguishes between rent for agricultural land and profits sought by industry. Whereas the former emphasizes the value of space or distance, as his formula shows, the latter places a greater value on profit or output. This leads Dunn to conclude that for agriculture, the production problem becomes one not of adjusting output to a marginal revenue line, but instead of adjusting the spatial location of production to a marginal rent line. As such, he refuses to accept rent as a function of output instead of distance in the case of agricultural production. Hence, space is explicit, as seen in Figure 2.

Still, tying rent value to yield per unit area and distance to market, as Von Thunen does, shows that it is possible to cultivate in the near vicinity of the market center crops that are both inexpensive per unit product and which provide high yields per unit land area, even though other variables that are traditionally tied to production location might suggest that such high-rent locations would be reserved for crops earning a higher market price per unit product. Moreover, by the same criteria, it is possible for crops that sell for more per unit product, and which are also more expensive to produce as well as relatively small in bulk per unit product, to occupy locations farther out from the market center, where land rent per unit area is relatively low. These spatial arrangements are more interpretations of the cropping pattern provided by the Isolated State, where low-priced yet bulky crops occupy locations near the market while more expensive items with less weight proved profitable even after transported long distances from the peripheries of the "state." Examples of these two categories of crops would be fire wood, butter, and dairy products other than freshly consumed milk, occupying the second and sixth zones of the Isolated 
State, respectively (see Figure 1). Regardless, these spatial arrangements rely on transport unit charges for the product, and on arrangements where land rent is calcuiatied per unit area.

Hall (1966) presents these Thunian arrangements in two general laws. The first states that with equal yields per acre (The Von Thunen assumption), the product with the lower production costs (per load) will occupy locations closer to the market center, since the value of land there is high, and vice versa. The second law states that the farther out from the market center that crops are cultivated, the lower per unit area their yield will be. Views similar to these regarding location versus productivity and the pricing of crops are presented by Jones et al (1978).

Given (a) the calculated net return for land rent per unit area, (b) the proportion-to-distance transportation costs, and (c) that all other factors are considered equal, as Von Thunen proposes, then it is shown to be more economical to cultivate crops that yeild more per unit area in locations in the near vicinity of the market, and to cultivate crops with lower productivity per unit area at sites farther out, since more remote sites provide savings in land rent with a not too much greater increase in transport costs. Considering such arrangements, the spatial distribution of crops that results, Von Thunen proposes, would not necessarily comply with market price per unit product for the commodities involved. Instead, as we have seen, higher net returns per unit land area would be the criterion for land-intensive crops such as the bulky grains, and would consequently draw lower priced yet bulky commodities closer to the market center, where land rents per unit area are higher. It should be pointed out here that the Isolated State is a prescriptive theory (Huff, 1981), that is, one in which the outcome is determined in advance according to a model or formula.

The spatial arrangement of land uses, according to Von Thunen, is such that the 
slope of the curve for the rent gradient (per unit area) rises smoothly and predictably as one approaches the market center, peaking at the very center of that market (see Figure 1). Laut (1968) elaborates on these findings, describing the resulting cropping pattem as follows:

A series of concentric zones of agricultural activities ... established around the urban market.... Ranking of these zones will be dependent on the economic rent each activity will provide, with the highest rents being nearest the city, those with the lowest being farther away from the city, with market gardening being assumed to provide the highest economic rent and beef cattle grazing the lowest. (p. 53)

Said differently, crop patterns and land uses in the near vicinity of the market center would appear as concentric zones engulfing the urban market. Beginning at the market center and moving outward these concentric zones of agricultural production would appear as follows (see also Figure 1):

Market gardening; forestry (for both firewood and lumber); crop farming without fallow; crop farming; fallow and pasture; three-field system; and, lastly, livestock farming bordered with stretches of wasteland fallow the year round separating the state from the outside world. (Grotewald, 1959:350; Johnson, 1970)

Isard (1956) ascribes these spatial arrangements to the fact that the market center occupies the optimal locations for land uses seeking higher profits. Rutherford (1969) agrees that production zones that are the function of one single variable, namely distance, would appear as simple concentric zones.

\section{THE INTENSITY THEORY OF PRODUCTION IN THE ISOLATED STATE}

In addition to the spatial patterning of crops in the peri-urban zone, there is another gradient that is predicted by the Isolated State--the production intensity pattern gradient. Because this theory is said to account for two gradients, it is frequently suggested that the Isolated State is in reality not one theory but two--one for intensity patterns and another for cropping patterns (Peterson, 1944; Horvath, 1967). In the words of Katzman (1974), 
"The Von Thunen paradigm comprises a theory of crop substitution and a theory of factor substitution or agricultural intensity" (p. 683-84). However, regardless of the degree to which these two theories remain distinguishable from one another, they remain highly interrelated. As Hall (1966) puts it, "[In Von Thunen's Isolated State] there is an intensity theory and a crop theory. But the crop theory is not a theory of intensity. It has indeed something to do with intensity, but the main factor is something else" (p. xxiv).

Regarding the intensity theory of crop production, Von Thunen applies it in two ways: (a) the added-inputs method, and (b) the crop/fallow cycle. In fact, though, Von Thunen applies a synthesis of both approaches in determining cropping patterns in the Isolated State. He calls the added-inputs method the "improved system," and applies it to locations in the near vicinity of the market. He applies the other, the crop/fallow cycle, to the "three-field" system at more distant locations where grains are generally cultivated. Von Thunen proposes that intensity rises most in the direction of the market center, and least toward the outermost peripheries. Such an arrangement is seen to agree with the slope of the rent gradient curve that Von Thunen presents as the criterion for revenues expected of land uses at different locations around the urban center; these also rise in the direction of the market center and decreases in the opposite direction (see Figure 2).

It is proposed by Von Thunen and accepted by subsequent writers that raising production intensity is one tactic farmers may use to combat rising rent per unit area in the near vicinity of the market (Hall, 1966:261). However, there is less agreement among writers on whether the slope of the curve for the intensity gradient drops smoothly as one moves away from the market, as is the case with the rent gradient, or if instead it falls as a broken line. Some writers (Hail, 1966; Peet, 1969; and Kellerman, 1977) suggest that Von Thunen's intensity gradient, unlike the rent gradient, does not rise steadily between Zone I and Zone VI, but instead drops. Other writers (Muller, 1973; Schlebecker, 1973; 
and Chisholm, 1968) suggest that the intensity gradient in the Isolated State drops rather smoothly throughout the model.

Regardless, it is a fact that higher intensity production is an effective device for combating higher land rent. As such it is safe to conclude that the intensity of production would rise in the direction of the market center, where land rent per unit area is highest. However, there are exceptions to this. Some crops occupy locations in the near vicinity of the market for the reason that they eam higher prices per unit product. Peet (1969) states the case as follows:

Some products [Von Thunen gives as examples fresh vegetables and milk] gain more from proximity to the market than others, and, within limitations set by their elasticities of demand, these crops "outbid" the others for the use of scarce inner land. Thus, Von Thunen says, "the price of milk will rise to the point where the land used to produce it cannot be more profitably devoted to any other product." (pp. 284-85)

On the other hand, locations closer to the market center occupied by some crops may be explained by factors other than higher intensity or bulkier production per unit area, even though the market price for these products is not as high. A good example of this is Von Thunen's firewood occupying Zone II in his pattern; it is known that firewood is produced at low intensity levels, especially with regard to the added inputs. Tarrant (1974) explains this situation as follows:

Since rent is calculated per hectare of the crops under cultivation, high yield crops like fire wood would incur high transport cost if cultivated far away from the market, since in the Isolated State the only means of transport was the horse and cart. This makes transport costs for bulky material like timber very high when calculated per unit area. (p. 24)

Tarant finds these costs to be higher than for wheat, although the latter is more intensively cultivated. He concludes that fire wood should he jroduced at locations with higher land rent relative to wheat production, which makes it occupy locations closer to the market center. Hall (1966) sees fire wood as representing a basic commodity with a highly inelastic demand curve and a permanent market to justify its production at higher rent sites 
in the near vicinity of the market.

Another situation, opposite to that for fire wood, would be the case of dairy products located in the outermost zone of the Isolated State, which relies on the less bulky final product (butter) to cut down transportation costs in the marketing of the diary product. Morgan and Munton (1971) present another explanation for the peripheral location of butter production. They see labor representing the largest portion of the total cost of unit production of butter, and they find labor costs declining in the same direction that transportation costs increase--that is, in the direction away from the market center-therefore offsetting the effects of higher transportation costs. In their view, then, it is inexpensive labor, and not inexpensive land rent, which accounts for the peripheral production of these dairy products.

As noted earlier, the intensity of agricultural production may be expressed in either of two ways. One is based on the frequency with which a given piece of land is cultivated as compared to another that is kept fallow (the crop/fallow cycle). The other is the addedinputs method, which measures the input of fertilizer, labor, pesticides, and the like. This added inputs approach is considered more an input/output ratio, and it is the method relied on in the discussion thus far.

One might be led to question the reason for employing two different methods for determining production intensity patterns. Hall (1966) explains the reasons for doing so as follows:

At sites nearer the market, it will pay to choose a more intensive system of cultivation in which a higher level of cost is associated with a later point of diminishing return .... Thus, intensification brings a higher rent, but as compared with the less intensive methods, it stops being profitable at smaller distances from the market (contrary to the less intensive method). (pp. $\mathbf{x x i x}-\mathbf{x x x}$ )

Still, Peterson (1944) warns that the productions rings in the Isolated State are not necessarily intensity rings. 
As was pointed out in the Introduction, the thrust of the present research is toward agricultural cropping pattems in peri-urban zones. However, since the intensity of production affects yields and retums from agricultural production, and since receptivity to higher intensity production varies among crops, it should be kept in mind that even when the research is limited to investigating cropping patterns only, the intensity level by which crops are cultivated continues to be an important factor in the yields per unit area, and, consequently, in the location of production as well as the rent per unit area that such production can bear.

\section{APPLICABLITY OF THE ISOLATED STATE TO PRESENT-DAY}

\section{PERI-URBAN AGRICULTURE}

Since the emergence of the Isolated State early last century, it has been much discussed and vigorously tested. Opinions vary widely on the theory's practical value--that is, its applicability to present-day peri-urban agriculture, particularly in economically and technologically developed regions where the majority of research is taking place (Heady, 1971). While some researchers find the theory applicable to today's peri-urban agriculture (Muller, 1973), others find it outdated (Grotewald, 1959). A third group finds the theory too specific, particularly insofar as it addresses a topic that must, they say, be approached on more general terms (Peet, 1969). A fourth group, however, finds the theory to be not specific enough; these feel that it does not lend itself to research applications insofar as it fails to identify specific zones for the production of specific crops, or, conversely, that it fails to permit the identification of specific crops with specific zones (Garrison and Marble, 1957; Jones et al, 1978). Some researchers might even be characterized as outright opponents of Von Thunen; these writers (for example, Robert Sinclair, 1967) completely reverse his intensity pattern and the attendant cropping pattern. Others might be considered 
to be loyal proponents, who support the theory, albeit with some modifications designed to accommodate circumstances brought about by developments in transportation and food-production technologies as well as changes in consumption patterns. One of these proponents is J. T. Schlebecker (1973), who maintains that the Isolated State is applicable to the circumstances of today's peri-urban agriculture in developed world economies.

Next to the work of Von Thunen himself, the work of Sinclair and Schlebecker represents the most comprehensive of the theoretical research efforts on the subject of periurban agriculture; this is so with regard to both cropping and intensity patterns. However, there is clear disagreement between the two on both of these focal issues, that is, patterns of crop production and patterns of production intensity. Consequently, discussing the views of these two prominent theorists will bring to the surface a great many new aspects, opinions, and viewpoints on the critical issues of cropping and intensity patterns in peri-urban agriculture.

\section{Schlebecker and the World Metropolis}

In his work entitled The World Metropolis (1973), J. T. Schlebecker argues for the applicability of Von Thunen's Isolated State to today's circumstances in peri-urban agriculture in developed economies, both for cropping and for intensity patterns; furthermore, he sees the historical evolution of American and world agriculture fitting well with Von Thunen's theoretical principles. He ascribes the continued applicability of the theory to the impacts of the city and to the nature of agricultural production, and he sees the following factors as influencing this trend.

First, he sees urban residents using legislation and land-use controls to manipulate the evolution of the countryside in a planned and orderly fashion, resulting in land uses and cropping patterns that best serve the interests (viz a viz consumption patterns) of the market population. At the same time Schlebecker sees farmers as ineffective instruments of change 
in the field of peri-urban agriculture for a variety of reasons, not least among them the fact that they lack the collective political power that distinguishes urban political entities.

Second, Schlebecker recognizes that some crops, by their very nature, are intensive users of inputs, thereby making them quite amenable to locations in the near vicinity of the urban market, regardless of the higher rent per unit land area. Other crops, however, are extensively cultivated and are far more economically cultivated at locations more distant from the urban center, where land rent per unit area is much lower.

However, Schlebecker cautions against the indiscriminate application of the Isolated State to today's peri-urban agriculture in developed countries. His recommendation is that the theory be applied on a macroscopic level so that the impacts of modem transportation and developments in food-preservation techniques may be accommodated. Furthermore, he sees such large-scale applications of the Isolated State to be more capable of ironing out irregularities in production patterns brought about by human actions, for example government programs and specialization of production. Regarding the shape of production zones, Schlebecker suggests they are shaped more like stars than concentric circles, as is suggested by Von Thunen, because their shape is determined most prominently by transportation lines that criss-cross the zones of production (see Figure 3, below).

Schlebecker qualifies his acceptance of the Isolated State in other ways as well. He recognizes that obvious changes have taken place since the early nineteenth century, among them changes in predominant fuels in household and factory use, consumer tastes, income, lifestyle, and a host of other particulars, all of which have their impact on the spatial distribution of agricultural production in peri-urban zones. Furthermore, he recognizes the large-scale effects of developments in transportation technology with regard to capacity, speed, availability, rates, and connectivity among outlying areas and transportability between them and the urban market at tolerable costs. 


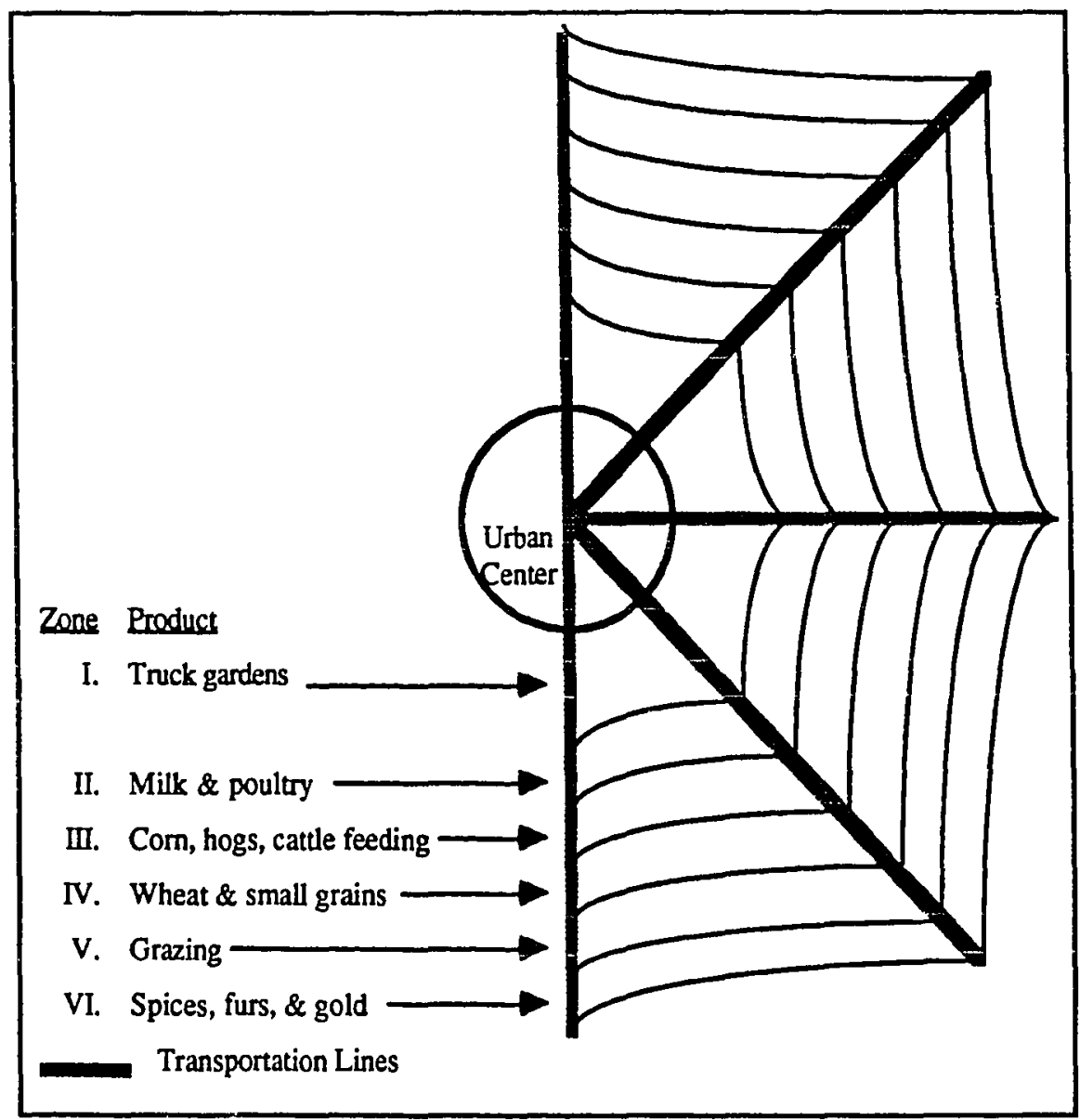

SOURCE: Schiebeciker (1973).

Figure 3. Land use patterns resulting from Von Thunen's agricultural cropping patterns, as modified by Schlebecker (1973).

Figure 3, above, illustrates today's peri-urban agricultural cropping patterns in developed countries as perceived by Schlebecker. In his pattern, Schlebecker suggests the presence of six consecutive zones of production. His zones are similar in number and kind to those proposed by Von Thunen, although they are shaped differently. Also like Von Thunen, Schlebecker assigns perishables production, which he terms "truck gardens," to Zone I of his pattern. Unlike Von Thunen, however, he permits this zone to extend deep into the countryside, unabated by the longer distances to the metropolitan market. This 
outward extension of truck gardening is due primarily to the availability of reliable and speedy transportation and to advanced food-preservation technology. These factors are emphasized even further in Schlebecker's assigning production of highly perishable dairy products, particularly milk, to Zone $\Pi$, which he does because of its need for large grazing pastures that are more commonly found at greater distances from urban centers, where land rent per unit area is low enough to make dairy production at these locations feasible, and yet where modern transportation and food-preservation techniques make the urban market accessible. Like Von Thunen, Schlebecker assigns grain production to Zones III and IV for basically the same reasons as did Von Thunen--that is, because of the extensive cultivation required for these crops and due to their low market piice per unit product.

Schlebecker summarizes his design of his pattern, and the extent to which it relies on many of Von Thunen's original findings, by asserting the following:

In spite of amazing improvements in transportation, the words "near" and "far" are not yet synonymous. Economic distance has not yet been eliminated by any governmental action. The establishment of zones of agricultural specialization still operates as suggested by Von Thunen. (p. 35)

The above implies Schlebecker's perception of the dominant effect of cities and their markets on land uses in their proximate peri-urban zones. It is interesting, however, that Schlebecker adds spices, furs, and gold to the outermost zone of his pattern, perhaps in order to arrive at the same number of total production zones as was originally suggested by Von Thunen. In this, however, is an echo of Grotewald (1959), who in order to retain Von Thunen's intensity pattern suggested replacing perishables production in Zone I with urban services, which is just one more indication of the interrelatedness and competitiveness of land uses in the peri-urban zone, the difficulty of putting solid boundaries between land uses, and the impossibility giving any of them permanent placement status. 


\section{Sinclair and the Theoretical Construct}

In his work entitled The Theoretical Construct (1967), Sinclair is highly critical of the Isolated State and of its indiscriminate application to peri-urban agriculture regardless of the degree of urbanization and level of technological development in the area to which it is applied. This view is based on his contentions that the theory's applicability depends on the precise identification of the factors responsible for the production and distribution of crops in a given peri-urban zone. What is lacking in Von Thunen's theory, in Sinclair's view, is specificity. Satisfying this lack makes feasible analyses of peri-urban agricultural zones, comparisons of contemporary peri-urban zones, and also comparisons across time periods. Sinclair asserts that recent developments in the fields of human organization, tastes, and habits, as well as achievements in the technologies of food preservation and agriculture have greatly influenced land-use practices in the peri-urban fringe as well as the processes of making agricultural siting decisions. Grotewald (1959) offers similar views on the effects of the changes that have occurred since the time that Von Thunen first proposed his theory, agreeing with Sinclair that these altered circumstances greatly curtail the applicability of the Isolated State. Consequently, Sinclair believes it is necessary to tie the application of the theory to the level of economic and technological development of the region under study. In short, Sinclair holds that more highly developed and urbanized circumstances obstruct the smooth application of the theory.

For example, Sinclair asserts that under highly developed conditions, the intensity of agricultural production will decrease in the direction of the market center, and finally vanish at the very center. This is so primarily because of uncertainties exacerbated by urbanization as well as other urban-related impacts unfavorable to agricultural production. This is, of course, precisely contrary to what is asserted under the Isolated State. Sinclair qualifies this assertion, however, limiting it to urban centers in the developed world, where 
detrimental impacts of urbanization like pollution, littering, and land speculation (i.e., the threat of displacement) actively discourage crop production in peri-urban areas.

Furthermore, Sinclair sees modem transportation as encouraging this trend toward less intensive agricultural production in the near vicinity of the urban market by easing access to more distant agricultural sites that offer beth lower land rent per unit area and better growing (environmental) conditions. Sinclair believes these trends to be a direct outgrowth of economic and technological development. Consequently, he views the Isolated State as applicable to peri-urban agriculture in less developed economies, and progressively less applicable in progressively more developed economies.

As a result of his critical view of the Isolated State, Sinclair proposes his own model of peri-urban cropping patterns. Sinclair's model is composed of five consecutive zones encircling the urban market (see Figure 4). The diagram presented in Figure 4 is not identical to that presented in Sinclair's 1967 article, since his is made up of regular concentric circles similar to the zones of production shown in the Isolated State. The figure below, however, more closely represents Sinclair's views. 


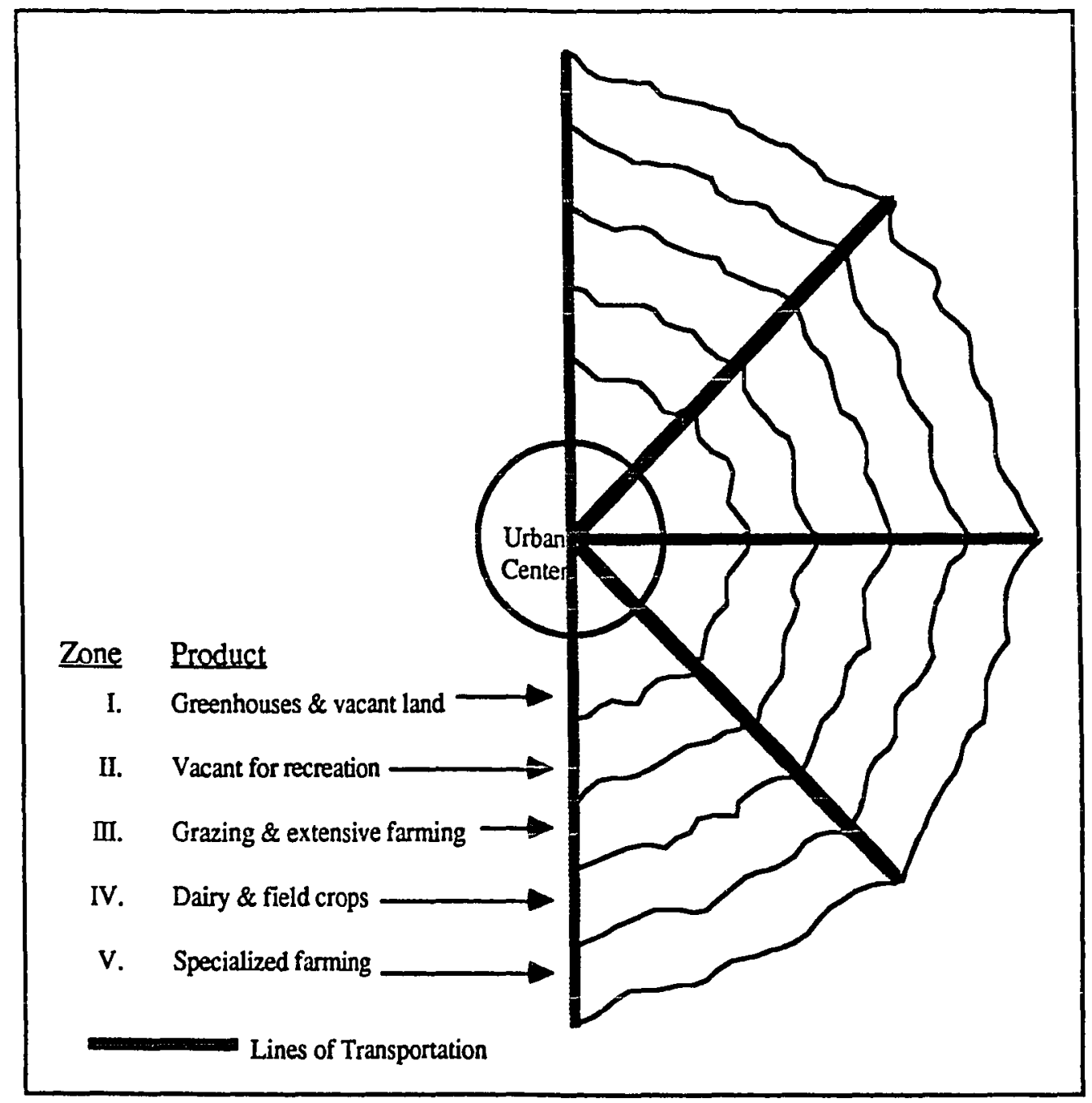

SOURCE: Sinclair (1967).

Figure 4. Land utilization in the peri-urban and extended zone as predicted by Sinclair (1967).

As shown in Figure 4, Sinclair's zones differ from Von Thunen's in that they are not quite circular. Their irregular, oscillating shape is a result of several factors, including the irregular growth of modern urban centers, environmental inconsistencies, human whims, governmental policies and regulations, and the impact of irregular lines of transportation that cross the region. His five production zones, counting from the center 
outward, are as follows: (I) greenhouse cultivations with much vacant iand; (II) vacant land for recreation; (III) grazing and extensive farming; (IV) dairy and field crop farming; (V) extensive farming. In Sinclair's model, extensive farming is limited to areas far outside the range of the detrimental impacts of urbanization, which Sinclair asserts deter agricultural land uses.

Sinclair cites several studies in support of his position. For example, he cites a study by Gottman (1961) on crop production in the northeastern U.S. megalopolis, and Lessinger's study (1956) on orchard replenishment in California, which was subjected to the pressure of advancing urbanization. Sinclair also reports on Krueger's study (1959) on Canada's "disappearing Niagara fruit belt." In reporting on these studies Sinclair highlights several findings and developing trends, including the dominance of factory farms in and around the megalopolis, the outward shift of dairy farming, the switch to short-term crops such as grain production at sites in the near vicinity of the urban market, and the spread of vacant land surrounding the cities.

Sinclair agrees, however, that not all kinds of agricultural production change with the approach of urbanization. Instead, he says, "where there are farming regions at the margins of expanding cities ... such as the cash grain regions" (p. 86), there is not a great deal that urbanization can change. Under those circumstances, Sinclair argues, farming will continue unabated in one form or another until immediately before it is overrun by urbanization. Furthermore, Sinclair sees farmers turning to this kind of short-term, machine-intensive agriculture (particularly grain cultivation) as one way to alleviate the detrimental effects of urbanization on peri-urban agriculture.

This last point is significant to the present research, since it supports the hypothesis that short-term field crops are able to occupy locations in the near vicinity of the market under certain conditions, specifically, conditions similar to those outlined here by Sinclair. 


\section{POINTS COMMON TO THE THREE THEORISTS}

\section{ON PERI-URBAN AGRICULTURE}

Despite obvious differences among the three theorists (Von Thunen, Schlebecker, and Sinclair) on the subject of cropping patterns and production intensity patterns in peri-urban agriculture, many of the fundamental elements of their respective models share certain common threads. A summary of some of the most important of these follows:

(1) All three models present peri-urban agriculture as consisting of several consecutive zones (between five and six) that engulf the urban market.

(2) Production zones in each of the three models are the result primarily of a single factor, be it urbanization, distance to market, or man himself, rather than a multifactor process as is the case in actuality.

(3) All three fail to incorporate the environment into their models as a major determinant of the spatial distribution of peri-urban agriculture.

(4) There is little if any mixing of noncompatible crops in any single zone in any of the models.

(5) All assign perishables production to the zone nearest the urban center, while limiting grain production to outer zones. One exception to this is in Sinclair's model, which admits "a little" grain in areas near the market center that are threatened by urban encroachment. However, Sinclair's ascribing a little grain production to such a high-rent zone does not reflect a belief that revenues from grain yields could ever compete satisfactorily with urban land uses that generate considerably higher revenues. Instead, grain production at these sites is seen as a temporary condition at sites at the threshold of urban encroachment which land speculation has rendered useful for little else. What is special about grain production of this kind at these locations, according to Sinclair, is that it represents a short-term investment, is frequently machine produced, and that it requires 
little capital to produce.

\section{OTHER VIEWS ON THE ISOLATED STATE}

Many writers on the Isolated State and present-day agriculture in peri-urban areas concern themselves not with the theory's applicability per se so much as with the degree of its applicability. Chisholm (1968), for example, examines the Isolated State on both the micro- and macroscopic levels for both developed and developing countries, and he finds that the microscopic level is more appropriate for checking the applicability of the theory. This is the case since its application on such a small scale tends to neutralize the impact of machinery and advanced technological achievements on the location of production, and at the same time highlights the influences created by the individual farmers, particularly with regard to the factor of distance between the fields of production and the market. Chisholm goes on to refute the notion that any single factor can be the sole determinant of the location of production, even if that single factor happens to be the distance to the consuming market. In Chisholm's words:

It is foolish to expect the single factor of distance--which is not even geographical distance but economic distance, related to transport charges-to account for all phenomena, or even always to provide readily discernable regularities. (p. 71)

Chisholm concludes by adding more factors as determinants of crop location, including such things as elements of the natural environment, socio-economic influences, and government policies.

Peet (1969) agrees that applying the Isolated State on a macroscopic level opens the door to spurious influences on the analysis, although he goes on to say that application of the theory on the macroscopic level does not completely undermine its applicability to present-day peri-urban agriculture. And yet Peet, too, adds additional factors or determinants that he posits affect crop location in the vicinity of the market, citing among 
these elements of the natural environment and new technologies insofar as they are reflected in the forces of supply and demand. This latter view is shared by Dunn (1954). In his research, Peet tested for the applicability of the Isolated State on the macroscopic level in the Northeast United States and Western Europe, which may conveniently be tied together (Huff, 1981).

Peet concludes that a combination of factors (among them competition from foreign production, the fact that farming is more mixed than monocultural, and the unbalanced distribution of bodies of water and land masses) combine to militate against the development of consistent Thunian production patterns in the test area, mentioned above. Still, though, Peet sees many advantages to applying the theory. He asserts, for example, that doing so helps tie together in a causal fashion diverse aspects of an agricultural issue which might not otherwise lend themselves to causal analysis. An example of this would be discussing spatial changes in economic activity in a given region at the same time one discussed that region's level of technological development.

Muller (1973), in testing for the applicabilty of the Isolated State to peri-urban agriculture, also conducted his analysis on the macroscopic level, but did so in the northern plains states of the United States. Muller's findings are positive and show "a persistence of concentric contours focused upon the megalopolis" (p. 237). Muller explains these concentric zones in terms of the distance-to-market factor, which he suggests has shaped American agriculture since the early nineteenth century.

Schlebecker (1973) shares Muller's views on the historical evolution of American agriculture, but does so in a fashion that suggests agreement with the implications of the Isolated State. For example, he differs with Muller insofar as the latter accepts the importance of deviations from the patterns advanced by the Isolated State, particularly in areas with large population concentrations. This opinion is shared by Kellerman (1977), 
who also tested fo: the applicability of the Isolated State. Keilerman's study analyzed American agriculture in 3076 counties in the conterminous United States; unlike Muller, however, he found the theory not applicable to today's peri-urban agriculture in developed areas. It is worth noting that Kellerman applied factors other than distance in his examination of the validity of the Isolated Staie; these "other" factors included such things as urbanization factors, characterization of the market, and elements of the natural environment. It should be noted, though, that in the modified version of the Isolated State, which Von Thunen presented in later years, he introducted more factors of production as determinants of crop location in peri-urban areas. The major reason for these later changes are grounded in increasing the scale of application of the theory to a macroscopic level. This new scale covered more land area, more settlements, varying environmental conditions, varied means of transport, and other factors (Hall, 1966:227). This further reinforces the significance of the scale of apjlication when judging the applicability of the findings of the Isolated State to any particular cropping pattern, or when attempting to determine which factors of production that might influence these cropping patterns.

Grotewald (1959) also finds the Isolated State not applicable to present-day periurban agriculture, but he ascribes this incompatibility to changes in factors of production and distribution that have occurred since Von Thunen's time. Grotewald asserts that these changes have rendered the Isolated State obsolete. In his own words Grotewald has this to say:

A theory is applicable only if its propositions are applicable.... [The Isolated State's] propositions regarding the production, transportation, and consumption of agricultural commodities are no longer applicable. Otherwise, there is nothing faulty in the theory. (p. 347)

Grotewald goes on to suggest that advancements in technology, transportation, as well as changes in tastes and patterns of consumer demand have all reduced the ability of the peri- 
urban areas to satisfy the consumption demands of the urban center. At the same time, however, these same changes have brought to the urban consumer easy and affordable access to more distant agricultural locations and, consequently, have reduced to a minimum the competitive edge once possessed by farmers cultivating crops in the peri-urban fringe of urban market centers. The result of all of this, according to Grotewald, is that fewer perishables are being produced in the peri-urban fringe, and this to the advantage of more distant sites where environmental conditions are more amenable to the production of these crops. Grotewald cites particularly the cities of St. Louis, Kansas City, and Chicago as illustrations of the findings and patterns he describes in his study.

It is worth noting, however, that Grotewald suggests that perishables production in peri-urban areas would be replaced with services, so that the intensity gradient would continue to rise in the direction of the market center, where it would finally peak in accordance with the views of Von Thunen.

Two additional points must be raised in regard to Grotewald's findings and conclusions about peri-urban agriculture and Von Thunen's Isolated State. First, although Grotewald promotes the production of more perishable crops at sites more distant from the urban market, he does not (for environmental and other reasons) ever specify any agricultural crops that might replace the declining production of perishables in this high-demand peri-urban environment, suggesting instead that urban services would fill the vacuum. This brings us to the second point, which is that the Isolated State is a theory applicable exclusively to crop production, and not to activities and/or land uses that originate in the urban areas themselves. This is, in fact, the theory's most distinctive feature. Accordingly, introducing urban services into a model wherein they share cropping pattems created by agricultural factors in the model in such a way that the theory's predicied intensity gradient is preserved undermines the very essence of the theory. In fact, 
doing so subverts the theory and converts it into a theory of location in a long series that concentrates on activities other than agriculture and which then addresses land uses under urban pressure, adopting an urban orientation, and considering agricultural variables only as an alternatives. It should be noted here as well that production rings in the Isolated State are not necessarily intensity rings (Hall, 1966; Peterson, 1944). 


\section{CHAPTER III}

\section{THE IMPACT OF TECHNOLOGICAI INNOVATIONS ON THE LOCATION OF INDIVIDUAL CROPS IN THE PERI-URBAN FRINGE}

\section{INTRODUCTION}

Von Thunen and subsequent theorists limit the production of perishable crops to the peri-urban zone closest to the market center for a number of reasons, including the very fact of their perishability, the impact of urbanization, the cultivation characteristics of some crops, as well as other reasons already touched upon. At the same time these researchers assign the cultivation of grain crops to locations more distant from the market center. More recent writers on peri-urban agriculture, however, many of whom are not constrained by any specific academic treatments of the subject, have contradicted these theoretical assertions. Consequently, more recent writings on the subject of peri-urban agriculture has put forward views on the locations of various crops in the near vicinity of the urban market center that differ in various ways from traditional literature on the subject.

Examples of these new views on crop location in pri-urban zones include the finding that perishable crops are being cultivated at sites more distant from the urban center. This has become recently possible since transportation and refrigeration technology has virtually eliminated perishability as a limitation on the transport of otherwise "perishable" corps. At the same time, grain cultivation is also no longer viewed as resiricted to outlying regions, as is suggested in the conventional literature. This new development is being introduced by writers who are challenging the "extensive-cultivation" argument that has for 
decades been used to explain why grain crops in fact were limited to outlying sites.

It is clear that many of the changes being noted in the locations of crops around the urban market are the result of new sets of factors, primarily those resulting from technological innovations, which are influencing agricultural production and its siting in ways much different from the influences of only a few decades ago.

The following discussion shall focus on just two crop categories--grains and perishables--and shall look at recent spatial arrangements of cropping patterns for them as well as at the factors most prominently influencing these patterns.

\section{CHANGES IN THE LOCATION OF PERISHABLES PRODUCTION}

\section{RELATIVE TO THE MARKET CENTER}

The literature on peri-urban agriculture indicates that perishables production in developed world economies occupies two primary locations--sites in the near vicinity of metropolitan centers, and sites farther outside them (Symons, 1967; OECD, 1979). The locations of the close-in sites can be explained in terms of their accessibility to the market; however, the newer, more distant sites must be explained in terms of the increased access to better food technology and modern transportation that makes more feasible the growing of perishable crops greater distances from the market, where better environmental conditions and lower land rent per unit area, respectively, enhance yields and increase net revenues.

Chisholm (1968) maintains that cultivating environmentally more suitable locations results in earlier harvests, which in turn command higher prices; this increased income, then, offsets the extra transportation costs incurred by cultivating distant locations. Grigg (1969) shares this view, and adds that cultivating crops in environmentally more amenable sites reduces production cost per unit product and enhances productivity per unit area, both 
at no increase in cost per unit production. Johnson (1969) asserts that the greater distances for transporting goods produced at more distant sites is easy to accommodate by means of new technologies and modem transportation. These influences are so strong, in fact, according to Johnson, that it is becoming both logistically and economically feasible to market Colorado's lettuce in Boston, thousands of miles from the point of production.

This outward migration of perishables production is also upheld by Grotewald (1959), who asserts that vegetables production in the vicinity of major midwestern cities has lately been declining in favor of more beneficial distant cultivation sites. In support of this view, Grotewald cites the cities of Chicago, St. Louis, and Kansas City, where he has observed and verified this trend. Grotewald explains the locational shift of perishables production by citing a low-cost transportation system connecting the Midwest with out-of-state locations where production conditions (particularly those pertaining to the environment) are more conducive to perishables production.

One major goal of cultivating crops where it is environmentally suitable is to satisfy the interests of certain categories of food producers who seek higher quality, more homogeneous, and more stable production. Examples of these food producers include chain stores and fast food businesses in the United States, which are rapidly increasing their share of the food market, particularly with regard to perishables, since both are interested in high quality, homogenous production. Moreover, the rise in competition between fresh and preserved food producers in many ways boosts the attractiveness of outer locations, where most foods targeted for preservation are supposedly cultivated (Woolverton, 1987). However, due to developed transportation, these outer sites are proving to be an acceptable alternative growing site for produce that is normally marketed fresh (Isard, 1956). Schoenemann (1987) discusses this trend as it pertains to the cultivation and marketing of potatoes: 
The supply side is only one dimension of potato marketing; the other ingredient is consumer demand. Over quite a few years the percentage of the crop sold in the fresh form has continually declined while the volume of potatoes used in processed forms has expanded. (p. 22)

Obviously, the situation with potato marketing applies equally well to a number of other crops that are conventionally consumed in both their fresh and processed forms. An additional factor is that processed produce is trimmed of its unnecessary portions, which reduces its weight and bulk, and in turn makes it more economically transportable. It should be noted in this discussion of the competition between fresh and processed produce that prices for fresh produce have in the past two decades risen more sharply than have prices for processed agricultural commodities. Miles (1977) points out that "between 1960 and 1972 retail prices for fresh perishable produce rose by 51 percent as compared to a rise of only 30 percent for processed fruits and vegetables, and a rise of only 32 percent for all cereal, bakery, and dairy products" (p. 42).

Krueger (1978) studied the locations of perishables cultivation in the Niagara fruit belt of Ontario, Canada, for the period 1931-1971, focusing on ten townships on or close to the south shore of Lake Ontario between the town of Hamilton and the Niagara River. He found that, except for grapes, the acreage cultivated with different fruit trees in peri-urban zones has been declining since 1951. At the same time, the number of fruit orchards has been increasing at locations more distant from the town of Hamilton. At the same time, he found that urbanization obstructed the replenishment of orchards in Hamilton's peri-urban areas, keeping production intensity low. This in turn promoted even further declines, therefore reducing its competitiveness against more rewarding urban land uses seeking those same sites. This trend has not held for the cultivation of grapes, however, and Krueger explains this fact in terms of the high resilience of the vines to varied environmental conditions, inciuding varied types of soil, which he ascribes to durable new European hybrids. 
The OECD (1979) cites both the truck and improved storage, as the major threat to perishables production near the urban markets. This is so, it is explained, because these new developments have "brought into the market a number of new suppliers whose costs are much lower. As a result, a shift is taking place in major producing areas away from growing agglomerations and sometimes to remote rural areas" (p. 27).

Schlebecker (1973), who has mych in common with Von Thunen and who assigns perishables production to the first zone of his cropping pattern, still permits this zone to extend deep into the countryside unabated by the lengthening distance to the market, primarily because of fast refrigerated trucks. Johnson (1969) shares this view.

\section{THE TWO-LOCATION INITIATIVE OF PERISHABLES PRODUCTION}

The notion of two locations engaged in perishables production, one in the near vicinity of the metropolitan center and the other farther away from it, is abundant in the literature dealing with developed world agriculture (Laut, 1968; Jumper, 1969; Chisholm, 1968). The OECD (1979) reports that up to 70 percent of the vegetables marketed in Vienna, Austria, in 1975 came from small holdings in the vicinity of the city. However, the OECD finds the truck and improved storage capabilities associated with more distant production areas to be major threats to perishables production near the urban markets. Otte (1974) reports that about 60 percent of all vegetables sold in 1969 came from SMSAs, as did 43 percent of the fruits and nuts. Jumper (1969) explains the situation as follows:

Traditionally, vegetable farms in the United States have been classed as either market gardens or truck farms. The immediate environs of large cities are favored locations for market gardens, which consist of small intensively cultivated plots producing a wide variety of vegetables for nearby urban markets. Truck farms, on the other hand, locate some distance from their markets, contain relatively large acreages, and specialize in a limited variety of vegetables. (p. 311) 
Symons (1967) presents a number of characteristics about these two sites of perishables production, and uses the same terms to denote them. Urban demand for perishables is satisfied from both locations, and not from one alone. There is a trend toward higher intensity production in the near vicinity of the market in order to compensate for higher land rent in these locations. Further, outer regions produce their crops earlier in the season, which brings higher revenues. She cites in this regard agricultural areas along the English Channel, the coastal lowlands of France, the Low countries, the Rhone Valley, the North African Coast, the Southeast coastal plains of the United States, and regions of drier climates farther west, notably those in Colorado and California. Additionally, Symons counts irrigation as a major factor in the cultivation of perishable crops in outlying regions and a stabilizing force for tetter quality produce.

Murphey (1982) also plays down the impact of linear distance to market on "perishable truck crops" cultivated under suitable environmental conditions at sites more distant from the market, since urban areas can afford the extra costs associated with marketing fresh produce. He cites Holland and Denmark, which possess sandy soils that produce early in the season and therefore command premium prices in neighboring European markets. Murphey sees a similar situation developing in the U.S., "with its highly developed system of fast, low-cost transportation and country- wide competition from fruit and vegetable crops grown in Florida and Califomia, [which] limits the scope of market gardening around cities in other parts of the country" (p. 146).

Chisholm (1968) describes perishables production in England as occupying two distinct locations as follows:

As to horticulture, a glance at a land use map of this country will show at once that there is a marked concentration of market gardening in certain areas of the country. Broadly speaking, the areas may be divided into those which are situated adjacent to major urban areas and those which are located where natural conditions of soil and climate are peculiarly favorable. (p. 82) 
This notion of the growing role of environmental condition in the locating of agricultural production is supported by the findings of a study conducted by the "glasshouse" (greenhouse) industry in Britain, which analyzed the costs of perishables production under various conditions. The study's findings "showed a net savings of over $£ 2$ million annually if all glasshouses [in Britain] were to be located in the climatically most favorable southern parts of the country" (Bennett, 1963:167).

Laut (1968) agrees with the above findings of his contemporaries, i.e., that outer regions enjoy a more specialized production which relies on a more cooperative environment and lower land rent per unit area. Still, Laut suggests that farms at inner sites can market their produce at low cost and on short notice, something that appears to be vital for many perishable products marketed as fresh produce.

Peet (1969) describes the use of outer locations for crop production as inevitable. He explains this outward migration primarily in terms of the rising demand for food in the urban markets, both quantitatively and qualitatively, which stems from the inadequacy of the peri-urban zone to satisfy the rising demands of the urban consumer either spatially, environmentally, or qualitatively. For example, Belkin (1985) points out that "a decade ago, the average supermarket carried 9,000 items; today it carries 22,000" (p. D 1). Jamison (1968) notes rising inventories in urban supermarkets as indicative of increased quantitative demand on the part of urban market centers.

\section{THE ROOTS OF THE DICHOTOMY IN THE LOCATION OF PERISHABLES PRODUCTION}

According to Furuseth and Pierce (1982), the dichotomy in the location of crops in the near versus the distant vicinity of the urban market stems primarily from the method by which the value of the land sites in these locations are determined. This is true largely 
because the assessment of urban land value involves a variety of criteria that include environmental, economic, and urbanization characteristics. Furuseth and Pierce put the matter as follows:

In considering the value of agriculture within an urban society, the initial task is to identify those lands which have the greatest potential agricultural value. This could be done by calculating the economic or monetary yield attainable from a parcel, or by using soil and climatic data to determine which land needs the least energy, fertilizer, and other external inputs and has the fewest environmental hazards. (p. 15)

The authors point out that the impact an urban area has on its surrounding fringe varies with the size of the urban center. In smaller urban centers, those with fewer than 50,000 people, peri-urban areas are more rapidly converted from agricultural to nonagricultural uses than are those of larger urban centers. The authors refer the phenomenon to the trend toward the decentralization of urbanization that began in the sixties. They maintain that this condition of more rapid turnover of land to nonagricultural use creates greater uncertainty about the future availability of existing agricultural land sites in these smaller urban centers, which discourages the use of these sites for perishables production. It should be noted here that although perishables production may be initiated on short notice, it does not flourish under conditions of uncertainty, because the cost of start-up is high and it requires a good deal of time to create a market for perishables produce, particularly where direct farm sales is a major business goal.

In fact, evidence suggests that perishables production is spreading fast in the peri-urban zones of the metropolises of developed world economies, too. Bowler (1981) ascribes this trend to three factors: "The wide spread of car ownership, purchases of deepfreezers, and increased leisure time" (p. 307). Woolverton (1987) verifies Bowler's view.

Consumers want good-tasting fresh vegetables. Consumption is on the rise. This has created a flourishing demand for fresh vegetables at farmer markets, roadside stands, and U-pick operations. It has also created a way for some farmers to keep a larger share of the consumer's retail dollars. (p. 15) 
Phipps (1983) holds that the incidence of perishables production at both near and distant locations can be explained because the same farmers invest in both areas in order to augment the natural environment and to overcome its limitations, depending on the circumstances at hand. This view has been stated previously by Krueger (1976). A case in point would be that of greenhouse culture found in peri-urban areas, which is designed to combat the inconveniences and incompatibility of surrounding environmental conditions with the cultivated crop.

Phipps recognizes the significance both of environmental factors such as soil types, topography, drainage, etc., and socio-economic factors such as the farmer's life history and his technical skills in the development of specific land uses. Still, he acknowledges that "there is little, if any, agreement as to the weight that should be given to physical and economic factors in the implementation of land use policies" (p. 61). Consequently, he advises against broad generalizations on this subject, suggesting instead that the issue be handled on a case-by-case basis. However, Phipps believes that with increased economic commitment, adjustments of established land uses become more and more stringent. He concludes that there are more factors involved in the production and spatial distribution of crops than one can count. Accordingly, part of the outcome of any analysis of land use patterns "may not be attributable to any known pattem. Furthermore, some influences attributed to socio-economic factors might be redundant with physical patterns and should therefore be reinterpreted" (p. 69).

The OECD (1979) suggests that not one but two opposing forces affect cropping and intensity production patterns in peri-urban areas--land rents and uncertainty. It is argued that whereas the first increases capital and labor commitments, the second reduces them. Accordingly, it is expected that different crops, as well as the same crop at different sites and under different conditions, would react differently to these two contradictory 
forces.

Jumper (1969) agrees that more than one factor determines the sites of perishables production, for locations both near or far from the market center. Nonetheless, he sees the major determinant of the actual cultivation site to be the quality, availability, and cost of transportation. Thus, Jumper argues that environmental suitability is not the sole determinant of the siting of perishable crops any more than is the distance-to-market factor. Instead, individual siting decisions are made in the context of alternative packages, since each choice would only satisfy part of the producer's needs.

This theme of the interrelatedness of the factors of production is sustained by Murphey (1982), who encourages a broad-based approach to determining the most prominently operative set of factors in agricultural production patterns:

Physical factors do not operate alone and may often be of relatively minor importance. The significance of location and of spatial relations especially is clear in accounting for land use and may often override the importance of local soils. (p. 146)

Murphey ranks both technology and demography ahead of soils as factors determining crop location. He perceives the relationship between market gardens and truck farming as competitive instead of complementary, with market gardens exploiting their near proximity to the urban market while truck farming counters with more suitable environmental conditions while still providing low-cost transportation. In Murphey's words:

Market garden areas are distinguished from truck gardens or truck farming because they are right next to the city, whereas truck farming may be carried on wherever transport gives it access to a market at bearable cost. Market gardens have to use what soil they find in the city environs, but even if the soil is poor, nearness to the market outweighs most soil disadvantages and makes it profitable to invest in soil improvements. (p. 146)

Murphey observes that Florida and California provide illustrations of exploiting on a national scale geographical locations relatively distant from the primary national market (the northeast megalopolis), wherein they enjoy the environmental and economic advantages of 
their locations, while modern transportation systems still provide profitable access to the market.

\section{THE FUNDAMENTAL QUESTION}

The discussion thus far indicates that more perishables are being cultivated at sites that are distant from urban centers than ever before, and that this is so primarily in an effort to exploit environmental and economic advantages (i.e., better growing conditions and lower rent), as well as to avoid the adverse impacts of urbanization. Furthermore, it has been shown here that the application of new technologies and modern transportation, among other things, has greatly reinforced this outward migration of perishables production. These developments, then, raise the important question of whether the forces that are currently promoting the out-migration of perishables production are strong enough to completely uproot remaining levels of perishables cultivation in the near vicinity of the urban market. If the answer to this question is "no," then one must follow-up by asking what force or forces are at work to preserve the cultivation of perishable crops in the peri-urban zone.

Finding the answer to this last question is a major goal of the present research. This requires expanding the research beyond the issue of environmental necessities of basic crop production, and encompasses an investigation of not just that whole class of factors pertaining to urbanization, but also to characteristics of the perishable crops themselves. Included in such an investigation would be an analysis of marketing strategies that influence the location decisions for perishable crops.

A second and related issue is that of the locations of other crops relative to the urban center, particularly grains, which prior theory has limited to the most distant tiers of peri-urban cropping patterns. However, this theoretical location assignment has 
historically been made for reasons that are no longer as defensible as they once were, again, largely because of advances in the fields of agricultural and transportation technologies, as will be shown next.

\section{THE CULTIVATION OF PERISHABLES IN THE}

\section{NEAR VICINITY OF THE URBAN MARKET}

Central to this issue is determining the factor or factors that keep perishables production close to the market center, despite the many forces that actively push it away from the urban center. Among these are high land rent, the apparent adverse effect of urbanization, less than optimal environmental conditions, and many others.

The literature shows that perishables production occurs on an appreciable scale in the near vicinity of the urban market (Jumper, 1969; OECD, 1979; and others). Otte (1974), for example, reports that up to 60 percent of the vegetable crop sold in the United States in 1969 came from SMSAs, as did 43 percent of the fruit crop. Otte reports further that land under cuitivation for fruits, nuts, and vegetables in U.S. SMSAs during the decade 1959 to 1969 increased rather than decreased.

Otte divides agricultural activity occurring in peri-urban areas and divides it into two major categories--that which competes with nonagricultural land uses in the same physical areas, and that which complements such activities. He finds that crops of the first category have a more difficult time surviving the nonagricultural pressures that are building in the peri-urban zones. Furthermore, Otte sees the perishability factor itself as increasing the chances of siting these vegetable crops closer to the market center.

Blair (1980) reports that a significant portion of the perishable crop in England is cultivated in peri-urban zones, although this is deemed a mixed blessing. On the negative side, Blair points to the detrimental impact of urbanization on peri-urban agriculture, 
including factors like vandalism, littering, traffic, dogs, pollution, and trespassing. On the positive side, though, Blair sees the cultivation of perishable crops in peri-urban zones as benefitting crops that are vulnerable to long-distance hauling or to time lapses between harvesting and marketing. Cultivating the peri-urban zone conserves transportation resources and costs. Finally, Blair suggests that near proximity to the urban environment provides peri-urban agriculture with the managerial expertise it needs, enhances farm-based recreation, and increases part-time employment, since perishables farming lends itself more readily than do other kinds of farming to part-time commitments. He holds that urban and agricultural environments, as represented in the peri-urban zone, enjoy a host of activities that reflect mutual benefit and attraction, among them the provision of camp sites and other recreational activity facilities, $\mathrm{U}$-pick fields, direct sales of fresh produce to urban consumers, home-delivery, all of which are integral to the business of perishables production.

It is worth noting that Blair's study was conducted in Essex County, England, one of Britain's leading agricultural counties. He found there that between 1960 and 1973 full-time employment in agriculture declined 51.2 percent. Furthermore, during the same period the incidence of part-time employment outside the agricultural sector undertaken by Essex farmers rose substantially, a situation reminiscent of circumstances faced by U.S. farmers. The significance of these developments is that they open the door for perishables production in peri-urban areas, since this form of agriculture is relatively well suited to part-time employment for persons outside the agricultural sector.

Bowler (1981) also writes on agriculture in Britain, focusing on strategies for combatting the effects of the drop in market prices for British produce that occurred as a result of the dumping of low-cost produce in the British market by European Economic Community (EEC) countries. Bowler reports that one way British farmers devised for 
dealing with the situation was to reduce, if not eliminate altogether, marketing expenses for their produce. As Bowler puts it, they arranged to have "a portion of their produce... sold directly to the consumer, since the value added through the regular distribution of those commodities ... can be as high as $40 \%$ of their retail price" (p. 307).

Bowler describes several methods British peri-urban farmers employed in marketing their produce directly to consumers, including door-to-door sales, farm sales, family shops, roadside stalls, shop deliveries, and urban market stalls. However, Bowler specifies "pick-your-own" (PYO) as the most productive technique, and he sees this marketing technique spreading widely in the peri-urban zones of major British conurbations, particularly in those near areas noted for their quality fruits and vegetables. Finally, he reports that perishables producers in peri-urban areas do compete against one another to occupy locations closer to the market center, a trend that he terms "location exploitation."

The OECD (1979) agrees that cultivating peri-urban areas results in numerous locational advantages, including a shorter distance to market, access to a larger pool of farm labor, and the availability of highly fragmented sites that would otherwise be fit for few uses, if any, at locations removed to greater distances from urban-related activities.

Symons (1967) acknowledges that developments in transportation have helped to diffuse market gardening over wider areas, deep into the rural countryside. However, she finds that large cities retain greater amounts of horticulture in their immediate vicinity, which she ascribes primarily to the higher intensity production that horticulture achieves. This characteristic of production fits well with higher land rents found closer to the market center. Gregor (1957), who also associates perishables production with the peri-urban fringe, differs with others in attributing the association to land fragmentation, which land speculation in peri-urban areas helps to promote. This view is shared by the OECD 
(1979).

Jumper (1969) has a great deal in common with Sinclair (1967) regarding the latter's "theoretical construct" pertaining to the declining intensity of agricultural production as one nears the market center. Still, Jumper finds that perishables do not follow this model of lower intensity and are, in fact, produced at higher intensity levels the closer one approaches to the market center, especially in areas where land resources are in short supply, as in California. It is well known, of course, that urbanization's threat to peri-urban land in California is real. Instead, Jumper reports that Califomians cultivate these peri-urban fringes with perishable crops intensively, an indication of the stability of perishables production as a short-term investment.

Von Eckardt (1964) deals with the subject of perishables production in peri-urban areas in the northeastem U.S. megalopolis, where land is under extreme pressure from urbanization, and where farming is sophisticated relative to farming in rural areas. However, Von Eckardt explains peri-urban agriculture in this region more in terms of the nearby market's extraordinary purchasing power and the higher unit price that agricultural production can command. Von Eckardt, then, sees the production of perishable crops in these areas as influenced primarily by their commanding higher prices per unit product. This requires, says Von Eckardt, that among other things the producers must act as smart managers as well as competent farmers, selecting the proper crops for cultivation as well as managing its cultivation correctly.

Von Eckardt lists among the products most suitable to this metropolitan market fruits, tobacco, nursery stock, greer-house products, white potatoes, and other greenhouse specialties. He observes that the "center of mushroom production in the U.S. is in southeastern Pennsylvania, within overnight shipping distance of all large cities of the megalopolis" (p. 51). Von Eckardt acknowledges that competition for land uses is fierce in 
this area and that this competitive pressure forces agriculture to become more compact, intensive, and mechanized. Some growers, however, are yielding to this pressure and are relocating to more distant and less expensive sites.

Finally, Von Eckardt recommends agricultural zoning as a means of curbing the threat of urbanization on agricultural land in the northeast megalopolis, so that agricultural production will become more stable. This, in turn, should encourage, among other things, the cultivation of capital intensive perishables.

\section{SHIFTS IN THE LOCATION OF GRAIN PRODUCTION RELATIVE TO THE URBAN MARKET}

The argument regarding the disparities between theory and practice in the spatial distribution of perishables production is every bit as prominent for the spatial distribution of grain production. In the case of grains, however, the argument takes an opposite geographical, or directional, course.

Both Von Thunen (1826) and Schlebecker (1973) assign grain production to the outer zones of their cropping patterns, where land rent per unit area is much lower than at sites closer to the center. This, they claim, compensates for a number of factors, including the low market price per unit product which these crops earn, their low intensity cultivation per unit area, and their relatively high transportation costs per unit land area. For Sinclair (1967), however, in whose model the majority of grain production is assigned to outer locations where the impact of urbanization is least noticeable, there nonetheless exists a limited amount of grain production in the zone closest to the urban market. Sinclair, after all, views urbanization as a destabalizing factor in long-term agricultural land uses in the peri-urban zone, whereas grain production represents a short-term investment, requiring limited capital expenditure and relatively little labor. These factors make grain cultivation a 
suitable "temporary" investment for land that is threatened by imminent urban overrun. To reiterate, Sinclair makes it clear that grain production in the near vicinity of the market is only a temporary solution for sites on the verge of being overrun by nonagricultural uses, a point which the present research proposes to reconsider for reasons explained below.

Assertions by Von Thunen, Schlebecker, and others to the effect that grain production, which is acreage-extensive and commands a relatively low price per unit area of cultivation, is not suitable in the vicinity of urban markets where land rent is high, are becoming less and less convincing. In fact, recent literature on grain production questions one of the field's fundamental assertions, indicating that extensive production with low productivity per unit area is not the invariable condition for grain cultivation. Instead, as a result of advances in the field of agricultural technology (and many of the same ones that have allowed perishables production to move away from the urban center), grain production may be practiced more intensively than ever before, with yields per unit area more than double traditional levels (Schneider, 1986).

There are a number of ways to achieve such notable increases in productivity, and among these, research into environmental effects of the locating of grain production is proving highly rewarding. The Farm Joumal (January, 1986), for example, suggests that farmers could raise land productivity in the cultivation of corn to up to 160 bushels per acre from the present level of 100 bushel by, among other things, stepping up fertilizer and herbicide application, and, much more importantly, by redistributing plants into narrower rows. This, according to the Farm Joumal, would result in better soil utilization, an environmental factor. Smith (1986) agrees that more suitable environmental conditions are capable of raising com production to up to 200 bushels per acre, although he argues that doing so requires, among other things, planting corn in wider rows, which, despite its contradiction of the above, is again an application of environmental factors to production 
questions. Schneider (1986) summarizes some of the profound changes in grain production, summarizing the highlights as follows:

Com farmers in the Middle West will average a record 120 bushels an acre this year, nearly five times the average yield in the mid 1930s .... Farmers in the European Economic Community recently started planting a new winter wheat variety that has produced crops that are 20 percent larger than before. And several American companies have introduced new hybrid wheat varieties in the last three years [that] increase yields by 30 percent in some areas. (p. F2)

Looking exclusively at wheat productivity in the U.S., though, one finds startling variations in production, "ranging from an average of 75.0 bushels per acre in Arizona down to an average of 13.2 bushels per acre in South Dakota" (Heid, 1979:98; 1976 statistics). Baldridge and Bowman (1987) report even higher wheat yields than those reported by Heid; largely due to access to sufficient water supplies through a combination of rainfall and irrigation, Montana achieved productivity as high as 86.3 bushels per acre (p. 8; 1986 statistics). However, in the absence of such water supplies, average yields at these same Montana sites dropped to as low as 44.0 bushels per acre. It should be noted that these yield differences reflect intensity differences, too; furthermore, that they are so startling weakens propositions advanced by Von Thunen and others that grain production is exclusively extensive.

A similar argument could be made regarding grain pricing per unit product, which has been enhanced by the opening of intemational markets for grain exports. Ikerd (1985) reports the following:

Food grain exports almost tripled in the last 25 years. Feed grain exports have increased by four and one half times .... In 1983, feed grain exports were equal to 40 percent of total production compared with exports of only 10 percent of production in 1959-61. Food grain exports have risen to 65 percerit of production in 1983 compared to less than 50 percent in the earlier period. (p. 14)

Ikerd also reports that international marketing of perishable crops is far less significant than for grains. 
The U.S. Department of Commerce (1981) puts it even more directly: "Driven by expanding foreign demand, cropland harvested rose rapidly, reaching 353 million acres in 1980," up from 289 million acres in 1972. Table I, below, shows figures for U.S. wheat harvested, marketed, and exported in the years 1976-1982. The table also shows price supports in dollars, as well as the market price per bushel. The table shows that wheat exports have lately reached very high levels, as did the average market price. This is also true for price suppors paid by the federal government to wheat growers.

\section{TABLE I}

QUANTITIES AND PRICING OF WHEAT PRODUCED IN THE UNITED STATES FOR SELECTED YEARS

\begin{tabular}{|c|c|c|c|c|}
\hline Year & $\begin{array}{c}\text { Production and } \\
\text { Carryover } \\
\text { (in millions of } \\
\text { bushels) }\end{array}$ & $\begin{array}{c}\text { Exports } \\
\text { (in millions of } \\
\text { bushels) }\end{array}$ & $\begin{array}{c}\text { Price Supports } \\
\text { (national average } \\
\text { loan rate is } \\
\text { dollars/bushel) }\end{array}$ & $\begin{array}{c}\text { Average Price } \\
\text { (in dollars per } \\
\text { bushel) }\end{array}$ \\
\hline 1976 & 2807 & 950 & 2.25 & 2.73 \\
\hline 1977 & 3148 & 1124 & 2.25 & 2.33 \\
\hline 1978 & 2953 & 2031 & 2.35 & 2.94 \\
\hline 1979 & 3076 & 2158 & 2.50 & N/A \\
\hline 1980 & 2376 & 1514 & 3.00 & 3.91 \\
\hline 1981 & 2799 & 1771 & 2.20 & 3.65 \\
\hline 1982 & 2812 & 1509 & 3.55 & 3.53 \\
\hline
\end{tabular}

SOURCE: Chicago Board of Trade, 1980; 1983.

The figures in Table I highlight two findings, which at first glance may appear paradoxical. The first is that linear distance to the proximate market center should drop in significance, and this most likely to the advantage of regional, national, or even international markets largely because of the large share of exports compared to wheat sold within the U.S. This should be even more the case where one knows that the majority of the grain will pass through intermediate processing prior to its human consumption. In 
sum, these developments reflect the ability of the grain crop to carry extra production costs, whether from higher land rent per unit area, which characterizes locations in the peri-urban zones, or from greater distances to market centers, which generally are also transit points, be they seaports, rail terminals with grain elevators, or assembly points where grain crops are assembled for further processing and marketing in processed form.

The second of the two findings pertains to the market price for grain per unit product, which established theory assumes to be low regardless of market demand; however, grain price is seen to improve by the opening and development of international markets for grain exports. As we have seen, export markets have expanded dramatically in the past two decades, and one would expect, then, that prices will have risen correspondingly (see Table I). It should be added that acreage sown is generally based on the previous two year' yield/income. Moreover, agricultural acreage responds slowly to changes in market prices.

Based on the above two sets of findings, then, it is hypothesized that the location of grain production will not necessarily be limited to locations relatively distant from urban centers, since convenient environmental factors and access to more intensive production per unit area, among other things, will tend to rectify many of the inconveniences which near proximity to urban centers might create. Consequently, the feasibility of grain production in the near vicinity of many urban centers is seen as greatly enhanced.

The above undermines assertions limiting production of grains in peri-urban areas to the outer portions of the zone, and also promotes a new patterning of agricultural production in these peri-urban areas. In this new pattern, a new tendency to mix crops in a single zone is replacing the old pattems of zonal patterning of production. Moreover, size as well as characteristics of the urban market has a great deal to do with crop selection in the peri-urban zone. 


\section{CHAPTER IV}

\section{RESEARCH DESIGN AND CONSTRUCTION OF THE MODEL}

The present research addresses agricultural production intensity and cropping patterns in peri-urban areas of developed countries and the forces influencing them, particularly as this occurs for individual crops. The crops under study are vegetables, greenhouse culture, and wheat. Wheat production will represent grain production in the empirical analysis. The review of literature has shown that there exist variations between the findings reported by theoretical studies and by other recent literature written on this subject.

These variations from the theoretical expectations result largely from the impact of variables that earlier theorists on peri-urban agriculture failed to take into account; these variables narrow even further the discussion about the purported "new" locations for the cultivation of both grains and perishables at defined sites in the peri-urban zone. These "new" locations of agricultural production in peri-urban zones are at the heart of the present research, and are addressed in the two hypotheses this study is pursuing.

The two hypotheses of the present study recognize the sectoral patterning of crops in the peri-urban zone. This is obvious from the admission of grain production to the immediate vicinity of the market while at the same time defending the continued production of perishables in the same locations, albeit for different reasons. This fact makes it logical that single crops be addressed individually rather than as crop families, lending the research 
criteria more readily to empirical testing. It also raises expectations for more accurate findings. A detailed explanation of the author's two hypotheses at the core of the present research and a discussion of the research topic from the point of view of the literature in the field follows.

\section{HYPOTHESIS NUMBER ONE: THE LOCATION OF PERISHABLE CROPS PRODUCTION IN THE PERI-URBAN ZONE}

The literature shows that changes in the structure and pricing of transportation, as well as prominent advances in technology, do in fact make feasible perishables production at locations relatively distant from the market center. Still, even these drastic locational shifts stop short of overturning Von Thunen's spatial pattern of perishable crop production. Even in the wake of the past century's great changes in technology and transportation, perishables production has continued to be located primarily in the vicinity of metropolitan marketplaces, even in cases when environmental conditions in those locations were less than optimal for perishables production. This situation is a result, largely, of those criteria that historically tie perishable crops to the metropolis.

With better technology and transportation, more perishables may be produced at locations that are more environmentally suited to their production; these locations are characteristically rural and more distant from the urban market. However, lower land rents per unit area, lower transportation costs per unit product/mile, and greater yield per unit area (achieved as a result of improved environmental conditions) combine to of ìset the effects of the greater distance to the market.

Regardless of this trend, however, perishables production in the near vicinity of the urban market will not necessarily be eliminated, as one might expect it would be. Instead, it is hypothesized that perishable crops will continue to be produced in these peri-urban 
zones near metropolitan market centers due to factors other than those of the natural

environment. These are expected to relate to: (a) characteristics of the perishable crops, and (b) characteristics of the metropolitan areas. Although nonmetropolitan areas might see an increase in perishables production over time, this increase would not significantly displace perishables production presently taking place in peri-urban zones. This assertion restates hypothesis number one, and the present research intends to empirically test this hypothesis.

Those factors seen to encourage the production of perishable crops in the peri-urban zone include, but are not limited to, the following:

(1) The willingness of urban residents to pay higher prices for perishable goods in order for them to be obtained fresh. Statistics clearly show that the market price for perishables sold fresh is much higher than for the same crops sold frozen, dehydrated, or otherwise preserved. In fact, in some cases the price of the item fresh might be more than double that for the same items designated for processing (U.S. Dept. of Agriculture, Crop Reporting Board, 1980). Expressed somewhat differently by Miles (1977), it may be seen that "between 1964 and 1967, although 52 percent of commercial vegetable acreage was grown for processing, the 48 percent grown for the fresh market provided 71 percent of total farm value" (p. 42). Still, though, the market for perishable crops remains a delicate one, and its location relative to the market center is only one in a long list of circumstances that affects it.

(2) The higher intensity production of perishables relative to many other extensively cultivated crops helps perishables compete more effectively against nonagricultural land uses competing for the same peri-urban sites. As Miles (1977) puts it:

Competition for the land takes the form of alternative agricultural as well as alternative nonagricultural land use. Land will be used for vegetable and fruit production only if the crop is more profitable than soybeans, cotton, or some other commodity. Export demand or a vastly expanded agricultural industry will, therefore, have the effect of displacing some land for an alternative use. (p. 24) 
Such competition and resultant substitution of land uses in the market's vicinity applies to grain production as much as it does to perishables. Such substitution of inputs in land uses constitutes the basis of the new cropping patterns posited in this research

(3) The short-term notice and the low-level investment that production of many perishable crops entails suits locations that are threatened by imminent changes in land uses. This condition is characteristic of peri-urban areas. The condition of short-term notice, on the other hand, pertains to the time needed for many crops, particularly vegetables, to mature and yield, which for many vegetables requires only a few weeks. Beans, for example, require only a minimum of 64 days to yield. Cabbage, too, requires only 64 days, and Chinese cabbage requires just 55 days. Corn requires 64 days, cucumbers 62 days, eggplant 52 days, and most other vegetables are comparable (Harris Moran Seed Company, 1987). This issue of short-term notice should be distinguished from another time-related issue for perishable crops, one which is vital to its success as a business: the time needed for the new farm/business to become known to customers in the proximate market. This is important since direct marketing is such an important channel of farm sales, largely because direct sales eliminate the high costs of indirect marketing that peri-urban perishables farming can rarely bear (Woolverton, 1987).

(4) Land fragmentation in the peri-urban zone caused by urban encroachment, particularly land speculation addressed toward business uses other than agricultural, which results in small lot sizes that are suitable for little, if any, agricultural production other than perishables production (OECD, 1979; Gregor, 1957).

(5) That perishable products, contrary to the case for most agricultural output, may be marketed directly to the consumer in the form of on-site sales creates large marketing savings for producers, thereby creating cash flow through an entire season as well as inflating the profit margin (Blair, 1980; Bowler, 1981; Woolverton, 1987). The short 
distance to market adds to transport costs savings and makes it easier for producers to deliver fresh produce to the urban consumer without the application of preservatives (Klassen and Arthur, 1987). This relationship between the urban consumer and the perishables producer is expressed succinctly by Woolverton (1987):

[Perishables consumption] is on the rise. This has created a flourishing demand for fresh vegetables at farmer markets, roadside stands, and U-pick operations. It has created a way for some farmers to keep a larger share of the consumer's retail dollars. (p. 15)

Obviously, these economic and logistical pluses are more available to producers in the peri-urban zone, who are actively seeking out these advantages to offset the negative impacts of proximity to the urban market. Large metropolises offer more open markets and greater opportunities for face-to-face contacts between producers and consumers, circumstances that are reflected in fresher produce, lower prices, and ultimately higher revenue for the farmer, since the middleman's share, which is commonly quite large, is divided between the consumer and the producer.

(6) Since many farms in the peri-urban zone are small and in many cases are only partially self-supporting, additional income from part-time activities outside the agricultural sector is frequencly essentiai. "The economic situation of the smaller farms that make up almost three-fourths of the farm sector [in the U.S.] often depends on the availability of off-farm jobs opportunities" (Utah Farmer-Stockman, 1987, p. 9). This encourages producers to locate relatively close to an urban center, where jobs may be found. Moreover, cultivating perishables is relatively labor intensive, which adds incentive to locate near the urban market, where a labor pool exists. This is the case even for large corporate farming operations. Since perishables production sits easily with almost any schedule, it easily accommodates the needs of a part-time labor force of the kind characteristically found in the vicinity of an urban center. Perishables cultivation is extensively practiced as a hobby, to raise fresh vegetables for home consumption and/or for 
ornamental vegetation (Frowine, 1986), making it all the more suitable to semi-urban settings characteristic of the peri-urban zone.

(7) The literature indicates that the size of the proximate urban center has a great deal to do with the speed with which peri-urban land is converted to nonagricultural uses. Findings indicate that larger urban centers convert land uses to nonagricultural purposes more slowly than do smaller urbari ceniers (Furuseth and Pierce, 1982; Krueger, 1967). This condition favors perishable crops produced in the peri-urban areas near larger urban centers. Although perishable crops may mature within a short period of time, they still require a longer time span and a greater capital outlay to establish as a viable business, particularly if the producer depends to a large degree on direct sales of his produce, and if the endeavor is implemented on a large scale.

(8) Several of the negative aspects of large metropolitan centers (including such things as congestion, pollution, crime, etc.) have enhanced the proximate countryside as both a leisure site and as the site of commuter homes and second homes. This has created an outward movement of urbanites who have become available for part-time cultivation of perishable crops, particularly on a smaller scale. A second effect of this outward movement of urbanites is a growing market for direct-sales perishables in these expanding outlying residential areas.

(9) Eaton (1973) finds that in the peri-urban zone, the high costs of extensive greenhouses cultivation may be offset by the advantages of location near the market center. For example, greenhouse cultivation improves on natural conditions substantially, since it eliminates insect infestation and competition for food and moisture from other plants. Additionally, says Eaton, "greenhouse gardening involves the 'forcing' of plants into bloom at a time other than their normal flowering period" (p. 162). This enables the producer to command the higher market prices available to out-of-season products. 
Nonetheless, greenhouse cultivation is expensive, and the market itself and the characteristics of the crops influence which crops may or may not be economically produced in this way.

(10) Because cultivating perishable crops is relatively labor intensive, and because it is frequently carried on at small-sized lots where machine use is highly restricted and economically infeasible (particularly when yields are freshly consumed), peri-urban areas are favored for the cultivation of perishable crops insofar as these areas characteristically have available smaller-sized lots. On the other hand, perishables production would be encouraged on larger lots in peri-urban zones in the vicinity of a large consuming market with a large pool of labor, a stagnant economy, and/or high unemployment (Wohld, 1987). Similar views are expressed by Miles (1977), who sees perishables production near and within metropolitan areas as "a special case of [agricultural] dispersion, with the potential for solving several problems simultareously" (p. 74).

In summary, a great many characteristics of the urban market encourage producers at peri-urban sites to ignore prevailing environmental conditions and cultivate perishable crops. This is particularly true in the northeastem megalopolis of the U.S. Von Eckardt (1964) explains that despite better environmental conditions elsewhere, and often at lower land rent, peri-urban farmers stick to the vicinity of the megalopolis. And what inclines these peri-urban producers to concentrate on perishables rather than other crops is that, among other things, perishables production is short-term (Morran Seed Company, 1987). For this reason, even a crop failure can be replaced and offset so that an entire season is not wasted (Jumper, 1969). Quite often farmers in the peri-urban zone cultivate consecutive plantings of the same crop at several locations, so that fresh produce continues trickling to the urban consumer for the full length of the growing season. The market for fresh produce is always available in the megalopolis, so that when produced at high intensity 
levels market factors make it worthwhile cultivating perishables in the otherwise unsuitable near vicinity of the large urban market, even when costly greenhouse cultivation is necessary.

\section{HYPOTHESIS NUMBER TWO: THE LOCATION OF GRAIN PRODUCTION IN THE PERI-URBAN ZONE}

Although grains are often cultivated on large tracts of land, usually with the assistance of heavy machinery and are commonly destined for international markets (Cramer \& Jensen, 1985), grain cultivation is still correctly considered a short-term investment. This kind of agriculture is suitable for areas where there exists the threat of urban encroachment, as is frequently the case in peri-urban areas.

Grain products, whether destined for human consumption or animal feed, require intermediate processing and are not consumed directly (Heid, 1979), so there is no obvious immediate benefit to locating grain production in the immediate vicinity of the metropolis. On the contrary, the cultivation and production of grains under convenient environmental conditions is the primary need for grain production's survival amid the relatively higher land rents found at peri-urban locations. One might add Sinclair's view (1967) that the short-term nature of grain production is suited to the transient nature of the peri-urban zone. Recalling that the impact of urban centers on the surrounding countryside is largely relative to the size of the metropolis (Furuseth \& Pierce, 1982; Krueger, 1976; Miles, 1977), and that rapidly growing urban centers are said to create less stable land-use conditions in their peri-urban fringes (Furuseth \& Pierce, 1982), it is reasonable to expect that few crops besides perishables and grains could survive in the peri-urban environment. Consequently, it is reasonable to expect to find both crops sharing the same peri-urban areas, in some cases for similar reasons, and in other cases for different ones (see Figure 5, below). 
Accordingly, it is held that due to the greater capacity of present-day production and the lower costs for modern transportation, and due to the greater productivity per unit area that cultivation in environmentally suitable locations makes possible, expanding production of grain crops into peri-urban zones is feasible and sound (see Figure 6, below). Miles (1977) illustrates this well in the following:

Local weather and climate are the most important variables affecting food production. Despite advances in irrigation, the development of new seeds for use in arid soils, and other technological advances, the local weather, the amount of sunshine reaching the crop, the temperatures of both the soil and the air, and the amount of rainfall determine whether the farmer has a good crop or a bad crop, and the kinds of crops he can grow. (p. 25)

The same point is made somewhat differently in The Good Fruit Grower (Jan., 1987): "No matter how good the farmer may be, the yield of a field will not go above that allowed by the most limiting environmental factor" (p. 16).

In line with the above and with previous discussions (see Chapter III), it is hypothesized that the spatial distribution of grain crops in peri-urban areas having advanced transportation and technology is influenced more strongly by factors of the natural environment than by the linear distance to the market and/or the cost of land rent per unit area. This should be even more the case for peri-urban zones near nonmetropolitan urban centers where the pace of development is fast and land rent per unit area is not quite as high as on the fringes of large metropolitan areas (compare Figures 5 and 6).

Besides the input of convenient environmental conditions, higher productivity per unit area could be realized by utilizing the added inputs approach, i.e., more fertilizer, more irrigation, and greater use of herbicides and pesticides, among other things. However, added inputs raise production costs per unit product. Significantly, though, some recent research indicates that it is economically feasible to adopt the added inputs approach (see Chapter III, "Shifts in the Location of Grain Production"). Accordingly, greater intensity production per unit area should be seen as a broader theme behind the second hypothesis 
and its dealing with "new" locations of grain production in peri-urban areas.

\section{THE NEW PRODUCTION PATTERNS}

The review of literature could be summarized in the following points:

(1) Perishables production will continue in the immediate vicinity of the urban market, despite more perishables being cultivated at outer locations largely because of advanced transportation, advanced food technologies, and convenient environmental conditions.

(2) Grain production is not limited to outer locations, as much theory suggests, since grain crops may also be intensively produced and therefore compete for locations in the near vicinity of the market.

(3) Several crops may compete for the same peri-urban sites, though each for different reasons. The outcome of this is extensive crop mixing, which results in a sectoral rather than zonal/concentric cropping pattern. This also runs contrary to theoretical assertions.

(4) In this new sectoral cropping pattern that would replace the concentric/zonal pattern, in which there is considerable crop mixing, low-priced crops would be found occupying sites relatively close to the market center. The resulting cropping patterns will vary qualitatively with the size of the urban center. Whether the urban center is metropolitan or nonmetropolitan will also affect the cropping pattern in the peri-urban zone. Figures 5 and 6, below, show these findings graphically. 


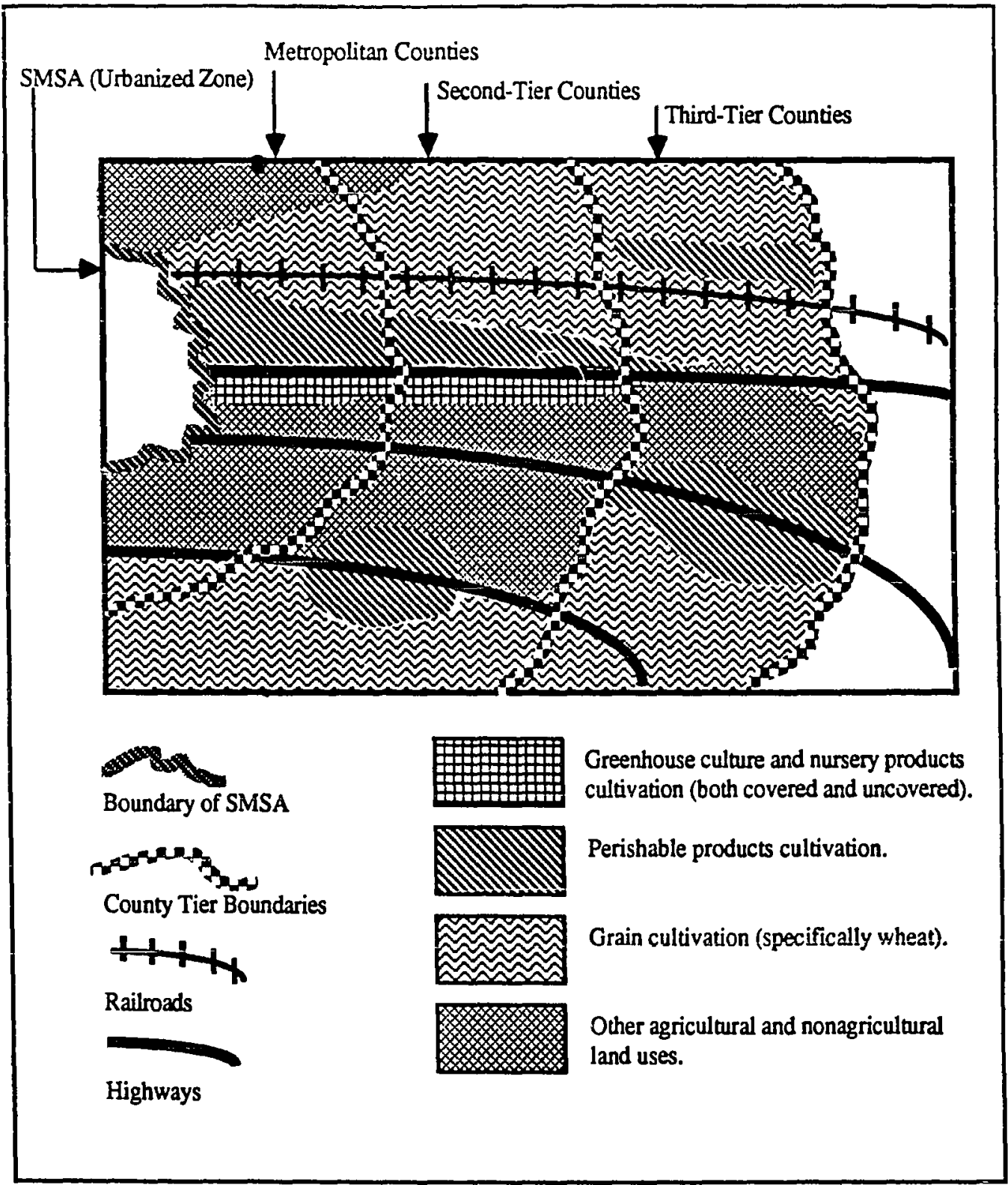

SOURCE: Author

Eigure 5. Agricultural cropping patterns in the periurban fringe of metropolitan urban centers (SMSAs). 


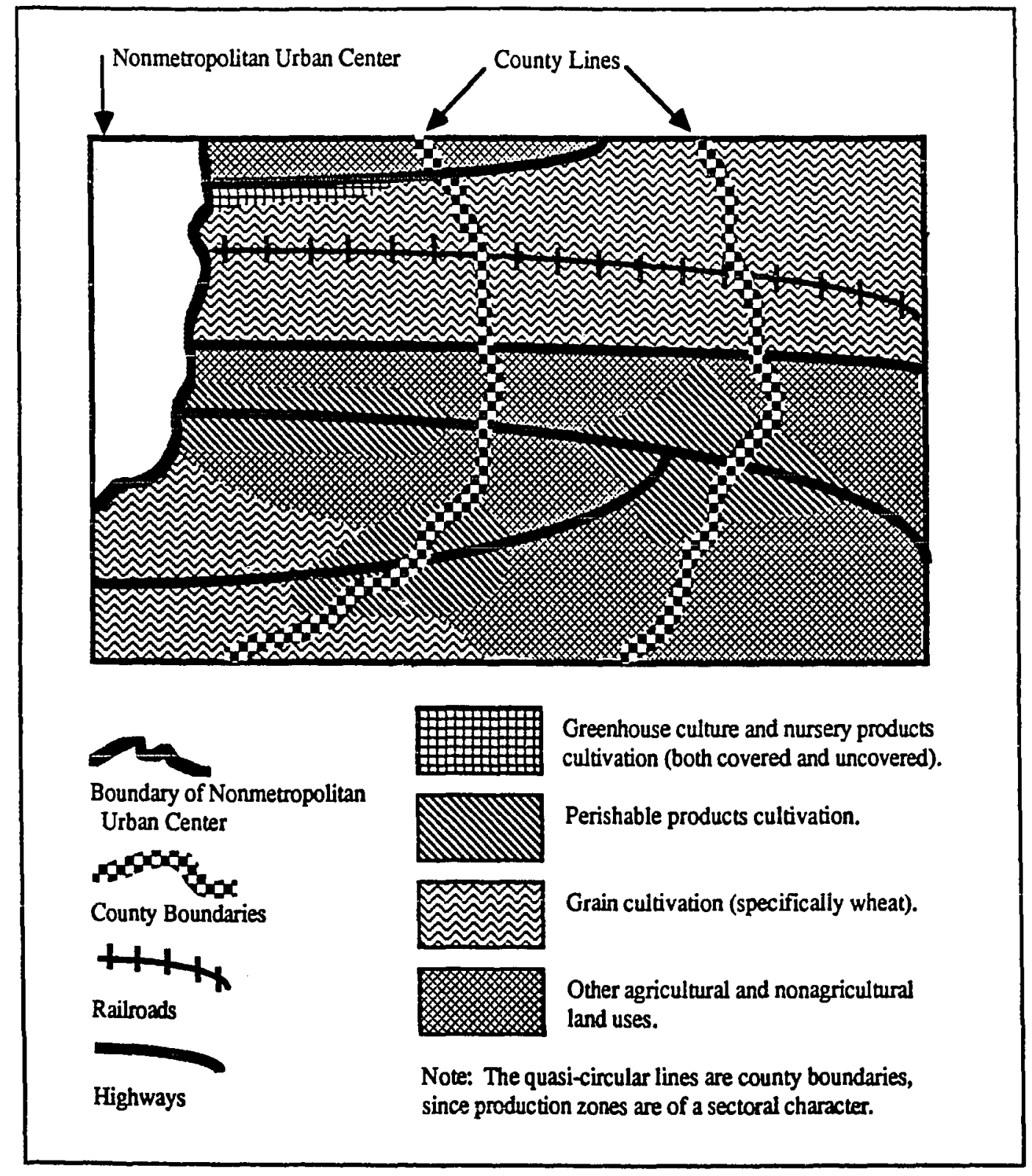

SOURCE: Author

Figure 6. Agricultural cropping pattems in the peri-urban fringe of nonmetropolitan urbari centers (with populations less than 50,000). 
Figures 5 and 6 present cropping patterns which this author sees as representing those found in peri-urban areas of developed countries. They reflect a configuration of the literature reviewed on this subject. Differences between the two reflect more quantitative than qualitative variations in land use patterns in these areas may be attributed largely to varied impacts of urbanization on the surrounding countryside. These impacts are affected somewhat by the size of the urban center, and are characterized by such activities as land speculation and other activities that affect the speed with which agricultural land is converted to nonagricultural uses.

The new land use patterns in these peri-urban areas are seen by this author to be sectoral and not concentric. This is seen to be the case because more than only perishable crops are capable of occupying high-rent sites at locations close to the market center. In fact, preliminary analysis of the data supports this sectoral patterning of agricultural production in the peri-urban zone.

It has been noted that the size of the urban center also affects land uses in the periurban area (Furusyth and Pierce, 1982). This may be explained as follows. Large urban centers are more stable and induce perishable production in their peri-urban zones. Smaller-sized urban centers have more dynamic centers, so that land in the peri-urban zone is subject to more rapid conversion to nonagricultural uses. Such highly unstable environments are seen to attract more short-term grain production and less perishables production. This conclusion is supported by the findings of the present research. Furthermore, larger, more sophisticated urban centers act as strong markets with powerful buying power, which is highly suitable to relatively expensive perishables. Smaller nonmetropolitan urban centers cannot match this significant economic force. 


\section{THE EMPIRICAL ANALYSIS}

\section{Area for Testing the Hypotheses}

The area chosen for empirically testing the research hypotheses is the so-called

"wheat region" of the United States. This area consists of 15 contiguous states, extending from Texas to North Dakota, and from Washington State to Ohio. These 15 states are the top wheat producers in the nation (see Figure 7). This testing area is to represent the status of peri-urban agriculture in the United States.

\section{The Rationale for Selecting the Wheat Region}

There are several reasons for selecting the wheat region over other agricultural regions for testing the accuracy of the research hypotheses. The second hypothesis intended for empirical verification is the spatial locations for the production of grain crops, specifically wheat, relative to market locations. The wheat region lends itself to this test for the obvious reasons. Second, it is posited that environmental factors more than any others account for the spatial distribution of grain crops (again, wheat in this case). According to this hypothesis, the effects of these environmental factors will override the effects of other infiuences, including those of urbanization and the consequent higher land rents in the vicinity of urban markets. Again, the wheat region lends itself to this analysis on the basis of its large area and the diversity of the climatic and environmental conditions that are found within it. The region is so large and varied in geography that a great many varieties of wheat are planted to best exploit each subregion's environmental profile. This variability in wheat-type production is so extensive that different parts of the wheat region commonly import and export their respective products to one another in order to satisfy varying local demand for specific wheat products.

Five primary varieties of wheat are grown in the United States (see Figure 7, below): hard red winter, hard red spring, durum, soft white, and soft red winter. These 
five varieties are cultivated in four major exclusive areas offering distinguishing environmental conditions, the most prominent of which are length of the growing season and the average annual precipitation. 


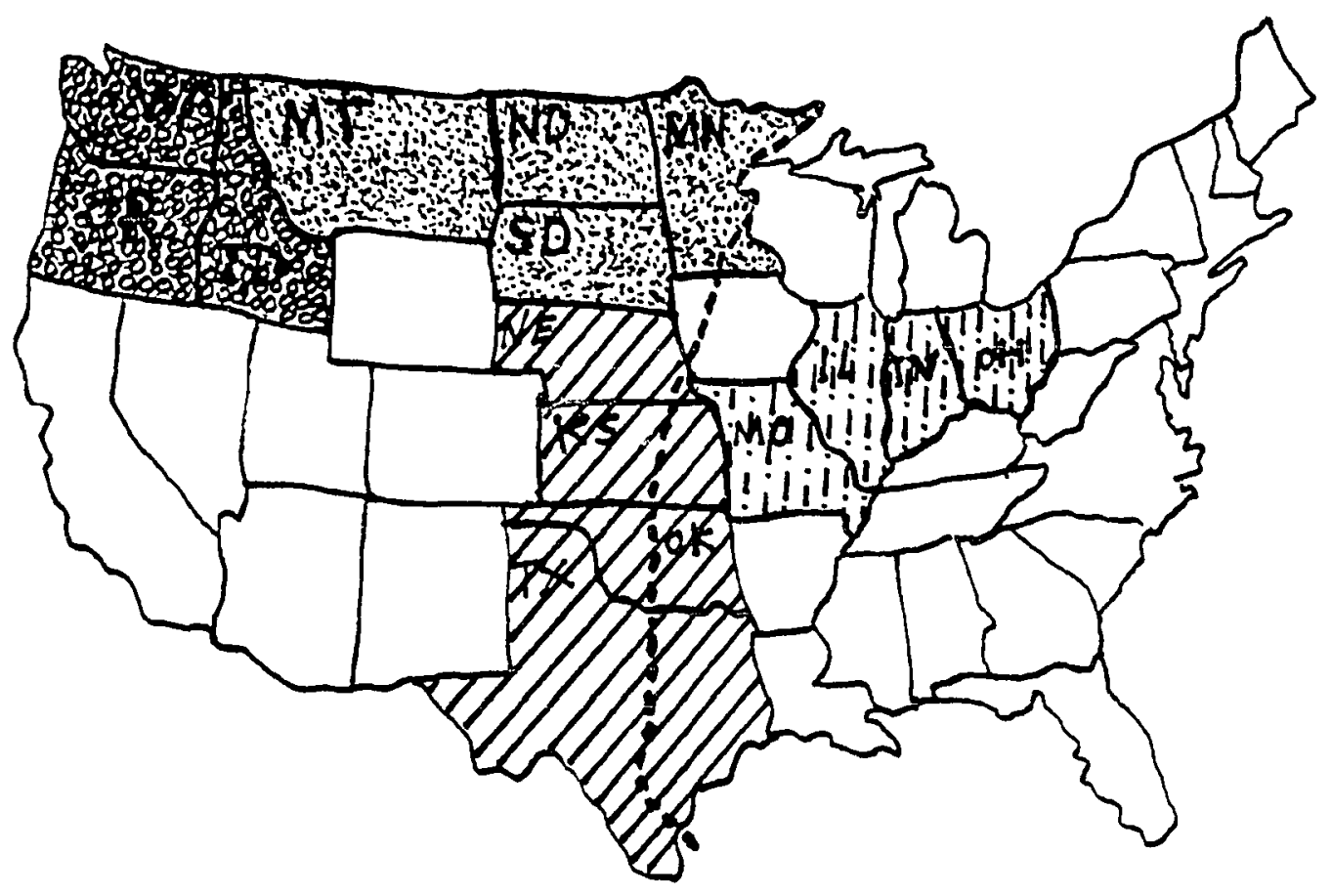

Q71 Hard Red Winter

Hard Red Spring \& Durum

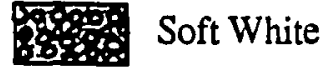

I!

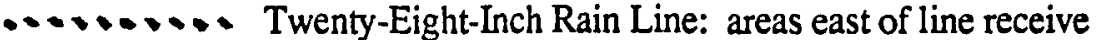 more than 28 inces of rainfall per year; areas west of line receive less than 28 inces of rainfall.}

Source: Heid, 1979; Zeitmetz, 1979.

Figure 7. The spatial distribution of the five primary varieties of wheat in the United States. 
The fourth reason for selecting the wheat region pertains to the first hypothesis, which deals with the location and production of perishables, specifically vegetables, greenhouse culture, and nursery production. In fact, the wheat region could be more suitable than any other region of the U.S. or any other developed country, primarily because it is not yet glutted with metropolises. Therefore, the rural impacts remain visible and continue to influence the location of crop production in their vicinity. Such impacts tend to originate in counties located farther out from metropolitan SMSAs, in tiers three and two. There are several hundred counties that satisfy these conditions from among the 1117 counties included in the present study. At the same time, however, this region contains a large number of SMSAs of metropolitan status (106 of them), which enables the research to measure whether or not a metropolis is capable of influencing land uses in its peri-urban fringe, particularly with regard to the location and production of perishable crops. It will be remembered that hypothesis one posits that perishables production is highly affected by the presence of urbanization, as well as by characteristics of the perishable crop itself.

\section{THE MODEL}

The method to be employed in testing the research hypotheses is Multiple Linear Regression (MLR). Applying MLR to the research data shall establish correlations between the dependent variable and the selected set of independent variables, that is, between the locations of crops cultivated in peri-urban zones in the wheat region and the various factors that have been hypothesized to be most influential in the siting of these crops.

MLR is well suited for testing correlations that are straightforward and of an additive nature. In the words of Heady (1971), "[MLR] is a versatile and flexible approach, provided we are concerned with linear and nonlinear phenomena; properly 
specified regression models are fairly realistic and computations are easily handled" (p. 249). Heady goes on to suggest that single-equation regression analysis of the kind employed in the present research has robust parameters obtained through least-squares calculations. In Heady's view, the robustness of these parameters is capable of bringing about valid statistical results even when the samples used are not drawn from a normal population. This differs from other models, which are highly sensitive to the relaxation of the assumption of normality. In fact, robustness of the MLR permits reliability even when assumptions in addition to normality are violated.

There are four sets of data (that is, four sets of counties to be analyzed); consequently, there will be four regression equations, one for each set of counties. However, in order to test for each of the three dependent variables, there will be three regression "runs" for each of the four sets of data, one for testing each of the three dependent variables. The four sets of data may be summarized as follows (see Figure 8):

(1) The set of aggregated SMSAs, which consists of the 106 SMSAs in the 15 states of the U.S. wheat region. This is Tier I.

(2) The set of metropolitan counties, which are those constituting the 106 SMSAs. These are made up of 253 metropolitan counties. These two sets are not identical. Variations exist between the two sets, and their comparison will amount to more than simply a data summation, since, among other things, urban impacts will vary with location and will not be limited to specific political jurisdictions. These differences will be highlighted in an analysis of both sets of data.

(3) The set of counties that immediately surround the metropolitan counties constituting the SMSAs. This is Tier II, which is composed of 467 counties that lie adjacent to the urban counties in the direction away from the urban center.

(4) Tier III is the set of counties that lie outward from, and adjacent to, the 
above-defined second tier of counties. This set consists of 291 counties (see Figure 8, below).

\section{THE UNITS OF ANALYSIS}

As implied above, the major unit of analysis to be employed in the testing of the hypotheses is the county, which is the official unit of analysis employed by the U.S. Bureau of the Census. Although census data will represent one major source of data for analysis, the present research is not limited to census data. A second primary unit of analysis to be employed is that of the Standard Metropolitan Statistical Area (SMSA); these SMSAs represent administrative "units" whose borders coincide with county lines. In most cases more than one county is included in a single SMSA. A further definition of SMSAs is provided by the U.S. Department of Commerce (1978):
A Standard Metropolitan Statistical Area (as defined by the Office of Management and Budget) is a county or group of counties that contain at least one city of 50,000 inhabitants or more, or "twin cities" with a combined population of at least 50,000 . In addition to the county, or counties, containing such city or cities, contiguous counties are included in an SMSA if, according to certain criteria, they are socially and economically integrated with the central city. The central city usually consists of the population of the city named in the title of the urbanized area .... Each SMSA must include at least one central city and the complete title of an SMSA identifies the central city or cities. (p. 1)

An interesting feature of SMSAs is that they are not static. Instead, they change over time with regard to the number of counties they represent (ranging from one to more than a score of counties). In addition, effective June 30,1983, the Office of Management and Budget, which administers statistics for metropolitan areas, changed the term SMSA to MSA (for Metropolitan Statistical Area), and revised the geographical limits of many individual SMSAs (U.S. Department of Commerce, 1986). These modifications, however, not just in the terms to be employed and their definitions, but in the geographical areas represented as well, should not be thought of as unusual, since the resident 


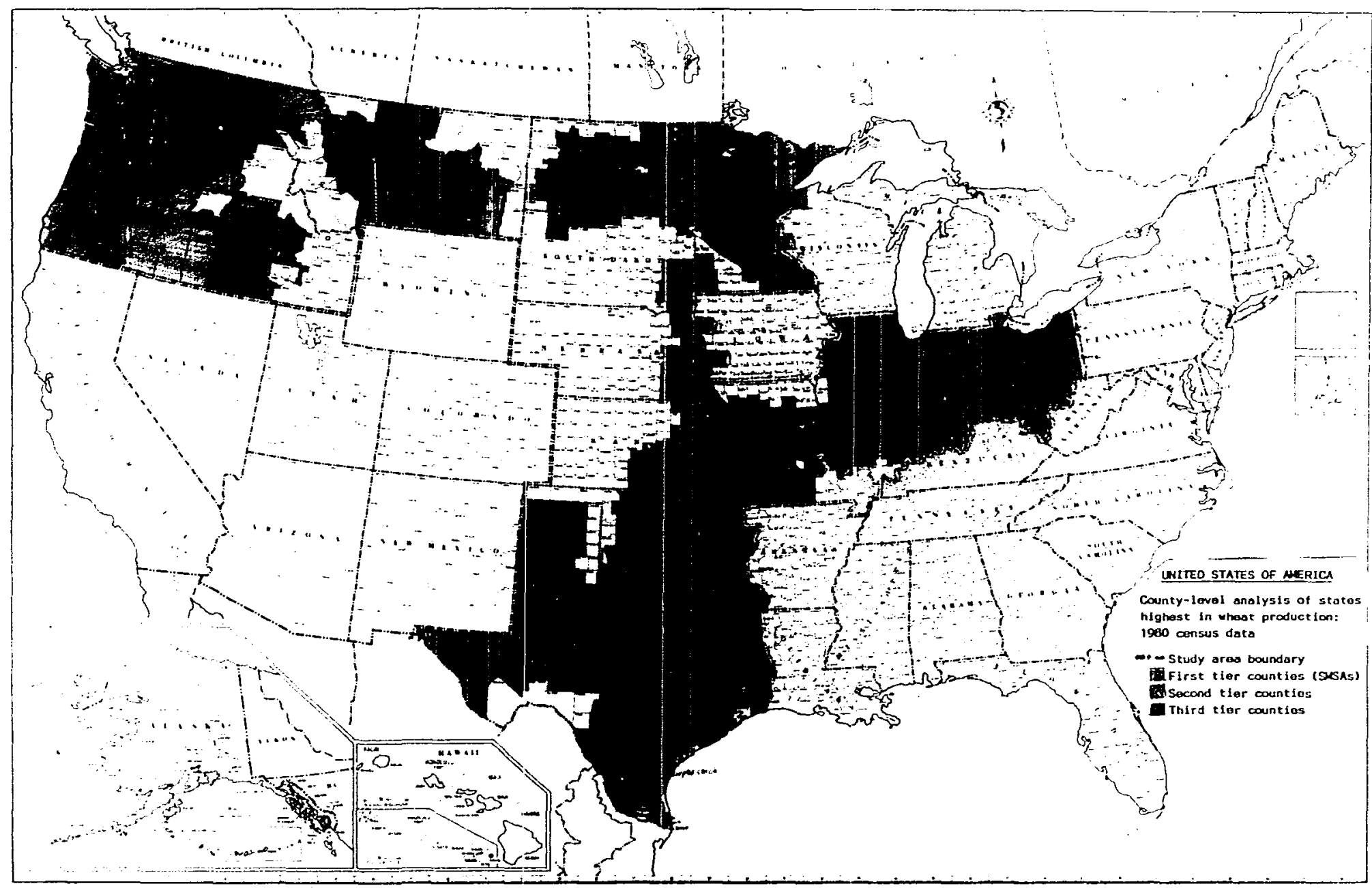


populations taken into account in the analysis is in a state of flux, itself changing over time.

\section{THE CASES}

The year 1980 has been selected for testing the research hypotheses. Census data for this year lists 106 SMSAs in the wheat region; these 106 SMSAs encompass 253 urban counties. Both the SMSA units and the county units will be used for testing the hypotheses; furthermore, two tiers of counties surrounding these SMSAs will be analyzed. By incorporating these three strata of counties into the analysis, the author hopes to test the impacts of nonmetropolitan urban counties on the spatial distribution of wheat, vegetable, nursery, and greenhouse cultivation in their surrounding vicinity (see Figure 8). Moreover, the impacts of nonurban areas on the spatial distribution of these kinds of agricultural production will be tested by analyzing the character and impact of rural counties surrounding the SMSAs and which are interspersed throughout the outer two tiers of counties. Implicit in this spatial arrangement is that outer counties entertain more natural environmental influences on the spatial distribution of crops than do inner counties.

\section{THE VARIABLES}

\section{Qverview}

The variables chosen to test the hypotheses encompass five general categories of factors: urbanization, transportation, the physical environment, characteristics of the individual crops, and characteristics of the marketplace. The selection of the specific variables is consistent with extensive reviews of the literature and a thorough analysis of the factors that most prominently affect the production and spatial distribution of crops. Although no set of variables is exclusive with regard to the question of factors affecting the spatial distribution of agricultural production, since this is such a highly complex issue, it is 
believed nonetheless that the present set of variables will satisfy the technical demands of the present analysis moreso than would any other set.

Paludan (1968) considers any set of variables to be useful in understanding agricultural land uses only if it includes variables that: (a) are external to the land being considered; (b) are internal to the land being considered; and (c) lead one to the answers sought. Paluden terms these three kinds of variables, respectively, "input variables," "status variables," and "output variables." Whereas a farmer cannot control status variables, he tries to manipulate the input variables in such a way that he achieves the desired output.

Similar views are adopted by Harvey (1966), who also identifies three primary factors of analysis: (a) demand for crops at specific markets, (b) transportation costs to those markets, and (c) the cost of different inputs (i.e., the input variables). For status variables Harvey lists such things as capital stocks and financial assets of the farm, yields of different products under different inputs, and the total land available for production. Lastly, he identifies output variables as decisions on production systems.

\section{The Dependent Variables}

The variables employed in testing the hypotheses of the present research consist of sets of dependent variables (also known as criterion variables), and sets of independent variables, predictors, or covariates. There are three dependent variables: (a) the harvested wheat acreage in each of the counties under study (WHEAT); (b) the harvested vegetable acreage in each of the counties under study (VEGET); and (c) the number of farms in each of the counties under study that employ greenhouse culture and/or nursery production (GREENH). Each of these dependent variables will be applied to each of the four data sets outlined above, resulting in a total of 12 regression runs. 


\section{The Independent Variables}

The set of independent variables, which it is hoped will isolate the factors most responsible for the spatial distribution of crops in the region under study, represent factors under the general categories of the natural environment, urbanization, transportation, the marketplace, and characteristics of the crops themselves. These categories of variables are drawn from the literature reviewed and they should present an accurate picture of the forces influencing the spatial distribution of the crops in question, insofar as they reflect the views presented in the literature reviewed. This view is summarized by Heady (1971): "The factors affecting agricultural output can be classified as ecological, economic, technological, and institutional" (p. 258). In the present research, the independent variables will include the following categories of factors:

(1) The Urban Factor. This category of variables represents characteristics of the urban environment present in the city/county being analyzed, although views vary considerably as to which specific variables best represent the urban factor. Taboda (1976) summarizes the difficulties of representing this category when he says that "[although] urbanization processes are among the most characteristic and significant contemporary phenomena, no universal and comprehensive indices of urbanization have so far been developed. The process covers complex economic, social, cultural, demographic, and technological transformations" (p. 75).

Brian Berry (1972), for one, considers the age of the city and the date when it achieved SMSA status to affect the rate of land transactions within it, and, consequently, the kinds of land uses to be found in its peri-urban areas. Other researchers consider the percentage of land area in the metropolis under urban usage compared to the overall land area of the respective county to be a factor affecting land usage in the peri-urban zone. Gottman (1961) acknowledges that the metropolis has influenced farmers' choices of crops 
in the peri-urban fringe; however, he admits that he has not yet figured out how to spell out these effects. Von Eckardt (1964) and Bochert (1972) both suggest that population size in the urban center presents an acceptable representation of the potential impact of that center on land uses in the surrounding countryside. This view reflects that adopted in the present research for measuring the impact of the urban center on land uses in surrounding countryside.

(2) Transportation. Another important variable is the accessibility factor (ACCESS), which is designed to measure the impact of accessibility to various means of transportation in the designated county on both the acreage cultivated and the selection of the crops to be grown in that county. Alexander et al. (1967) express it this way:

In a commercial economy having specialized transportation, the movement of goods is influenced by several forces .... The spatial difference in transport charges is not only a geographic factor influencing the circulation of goods, but also a geographic element in terms of which the character of a region may be expressed. (p. 552)

Due to the complex interaction of modes of transport, different means of transportation will be considered in the present research. However, they will be measured as dummy variables, with values ranging from zero to seven. The value of zero $(\varnothing)$ will be assigned to counties with no interstate lines, be they highway, rail, or navigable waterway; the value one (1) will designate counties with interstate highways only; two (2) will designate counties with interstate rail connections; three (3) will designate counties with navigable waterways; four (4) will designate counties with interstate highway and rail connections; five (5) will designate counties with interstate highway and waterway connections; six (6) will designate counties with interstate waterways and railroad connections; and seven (7) will designate counties with interstate highway, rail, and waterway connections.

Of note here is that more than half of the wheat produced in the U.S. is shipped to 
international markets using primarily unit trains and, to a lesser degree, water transportation (Johnson, 1970). This fact is an indication of the limited value (in the best of circumstances) of linear distance to locai markets in deciding the location of wheat cultivation. These claims are supported by earlier research that applies the linear-distanceto-metropolis variable and obtains quite weak correlations (Kellerman, 1977).

(3) Lot Size. This variable, (LOTSIZE), represents the average lot size in acres in the peri-urban zones of the county under study. Russwurm (1967) sees small-sized lots (a result of land fragmentation) as encouraging specialized production, mainly garden farming, at the expense of field crops. Moreover, he sees specialty crops as associated more with large cities. However, Russwurm also warns that since city markets can be supplied from outer areas, the significance of this assumed relationship may be greatly weakened, although not completely eliminated.

(4) The Natural Environment. This variable represents the environmental influences operating in the county under study. The literature on this issue shows no single factor predominating nor leading to any others; instead, the literature recommends selecting a representative factor or factors to cover for the many variables inherent in the environment. The process is explained here by Alcock et al. (1974):

The number of climatic factors operating in the atmosphere and the soil is large, and in practice it is often necessary or even logical to exercise some selection over what is to be measured. Determination of relevance depends on the biological response under consideration. (p. 66)

In this matter of selecting a representative or proxy variable, Gottman (1961) and Visher (1942) recommend selecting the length of the growing season. As explained by Arunachalam (1965), "The number of days of growing season free from abnormal weather conditions is a very important criterion affecting the distribution of agricultural cropping patterns" (p. 23). But this view is not universal. Grigg (1969), for example, suggests that it is best to use climatic criteria when analyzing large areas, but to use local morphologies 
when analyzing smaller areas. His point is that land potential is related to local economic and technical conditions as well as to the physical stability of the local soil or peculiarities of the climate. Cumberland (1964) seconds this approach, although he recommends that soil be looked at in conjunction with other environmental variables. Other researchers promote different variables for measuring the impact of the environment on the siting of agricultural production. Papadakis (1952) uses the availability of water during the growing season as well as the winter hardiness, the latter being a variable used by Heid (1979) and Von Eckardt (1964) to determine the environmental impact on the spatial distribution of crops.

In the present research, two variables are used to represent the environmental factor: (a) the length of the growing season in the county under study (GROWTH), and (b) the average yearly precipitation in the county (PRECIPIT). According to Arunachalam (1965), the term "growing season" describes the average number of days free of climatic extremities and which are satisfactory for the growth and nurturing of plants. The latter condition is generally measured in terms of temperature. Thoman (1968) estimates the length of the growing season for wheat that is developed for high latitudes to be 90 days free of abnormal weather. He considers necessary precipitation for wheat to be a minimum of nine inches per year. Alexander (1963) considers an average daily temperature of $43^{\circ} \mathrm{F}$ as a critical limit necessary for most forage grasses.

(5) Irrigation. The irrigation variable (IRRIGATE) represents the land area under irrigation in the county under study. The importance of irrigation in American agriculture is implied by Miles (1977), when he tells us that "about 36 percent of the entire national water consumption is used for irrigation. About 10 percent of U.S. agricultural acreage is irrigated .... Over half of our fruit crops is irrigated... [and] a third of the vegetables that we eat" (p. 60). In Miles's view, several factors accelerate the use of irrigation, among 
them the fact that moisture is a controlling factor in plant growth. Furthermore, irrigation represents a major step toward a more stable and predictable agricultural output, which chain stores, the most important marketers of food stuffs, strive to control. Added to this is the fact that irrigation and mechanization assist with production on a large scale, thereby reducing costs per unit product.

Among other things, then, irrigation facilitates agricultural production in outlying locations, where marketing of the product still requires additional transport costs. Moreover, irrigation encourages the intensification of production and increases productivity per unit area, circumstances that are essential to successful agricultural exploitation of peri-urban zones with their high land rents. This condition remains in force even for crops that are bulky and/or earn a low market price per unit product, which is the case for wheat (Baldridge and Bowman, 1987).

It is expected that the irrigation variable will correlate highly with land in peri-urban zones that is cultivated with any of the three crop categories under study. (It should be noted, though, that the Census Bureau withholds a good deal of data on the irrigation of individual crops so as not to disclose the identities of specific farmers.) As such, use of the irrigation variable will be limited to that applied to wheat and vegetable cultivation. The findings, therefore, which are expected to show a positive correlation between the cultivation of these two crops and intensity of irrigation, could be interpreted as indicating a means for pushing production of these crops in the direction of the market. This would be even more the case because locations closer to the market center have relatively higher land rent, which may be combatted by increasing yields per unit land by means of added inputs like irrigation. It should be pointed out, however, that the irrigation factor is expected to be influenced heavily by the presence or lack of other environmental factors, most prominent among these being the average yearly rainfall in the designated area. Since both of these 
factors will be substituting for one another, yet a third factor will be influenced by irrigation--the fertilizer factor.

(6) The Fertilizer Variable. The fertilizer variable (FERTILIZ) represents the quantity of fertilizer of different kinds that is used in the production of crops in the county under study. It is obvious that crops produced with the assistance of fertilizers cost more per unit product than those produced without; however, the use of fertilizers is a proven technique for raising productivity per unit area, thereby enabling agriculture to compete successfully with other nonagricultural land uses in peri-urban sites where land rents per unit area are high.

According to Schneider (1986), agriculture has passed through three revolutions, the second of which was incorporating "the widespread use of pesticides, fertilizers, and other farm chemicals" (p. 2), which took place following World War II. Accordingly, it is expected that the fertilizer variable will correlate strongly with crops cultivated in peri-urban areas, where high yields are needed to offset high land rents. This is expected to be the case in areas cultivating both perishable crops and wheat. One is led to conclude, then, that the fertilizer variable and the irrigation variable will work together to enhance and intensify agricultural productivity, particularly in locations in the near vicinity of population centers, where land rent is higher than in more remote locations. Still, the application of fertilizers is highly dependent on the availability of sufficient moisture, since adverse outcomes result from applying fertilizer to crops under dry conditions. The Good Fruit Grower (Jan. 1, 1987), for example, reports the following:

When farmers applied enough nitrogen fertilizer to produce 80 bushel yields but only had enough available water for 60 bushels, wheat plants tended to use up available water too fast, causing stress to the plants. Stressed plants were exerting suctions up to 700 pounds per square inch to pull water from the soil. (p. 16)

According to the same source, stress in plants increases their vulnerability to disease. 
Ruthenburg (1985) ties moisture stress in plants to retarded growth, including a reduced response to fertilizer inputs (p. 47).

(7) The Interaction Variable. This variable (INTERACT) represents the spatial interaction among diverse factors in the peri-urban areas under study, and it may be approached from two distinct points of view--that pertaining to interaction of SMSAs with the surrounding peri-urban zones, and that pertaining to interaction of nonmetropolitan urban centers with their surrounding peri-urban zones. For reasons explained below, different means must be employed for measuring interaction in each of these locations.

Spencer and Stewart (1973) suggest that any system of agriculture maintains a strong relationship with the structural makeup of the culture and society that employs it. This fact illustrates the need to examine factors beyond the inherent elements of agriculture itself, which leads to typologies and regionalism. Instead, Spencer and Stewart count three attribute complexes of cultural systems they see as shaping agricultural patterns. These are "organizational constructs," "economic conceptualisms," and "technological assemblages." Richter (1975), in line with these views, suggests the following:

The land development pattern is a result of the natural conditions of an area in question remodeled by its present and past use. This pattern, formed by natural, social, and technical factors, is a spatial expression of the dialectic interrelationships between nature and society. (p. 95)

Phipps (1983) agrees that land use choices depends on, among other things, the economic and social environment, the farmer's culture and history, and on the level of the farmer's technical skills. Klages (1949), however, warns that the social environment includes a variety of factors, so that, consequently, "the field to be considered under the social environment cannot well be circumscribed" (p. 58). Accordingly, a selective approach should be used in selecting variables to represent the social environment so that the whole group is represented in the most reliable manner possible. Russwurm (1967), for example, views spatial interaction as the percentage of land rented. He also measures it as the 
percentage of land improved.

For the purposes of the present research, the variables to be employed for measuring the spatial interaction are the population of the proximate urban center, and the nonrural population living in the peri-urban fringe. These population variables are seen to influence land speculation as well as agricultural land uses that serve the interests of the proximate urban population. As such, these population variables are expected to correlate strongly with the dependent variable representing the vegetable crops and with that representing greenhouse culture and nursery production. This is supported by the view that posits a positive relationship between the degree and quality of urban interaction with the countryside to the size and therefore the sophistication of the urban center, since this is seen to determine the kinds of land uses that are likely to occur in these peri-urban areas. Of note is that a higher level of urban interaction with the countiyside might activate land speculation and short-term cultivation of land, which would be expected to encourage the cultivation of short-term grains, including wheat.

In that portion of the present research that deals with SMSAs, the interaction variable (INTERACT) shall be represented by the urban population living outside the main urban center of the SMSA. In that portion of the present research dealing with nonmetropolitan urban centers, or consolidated SMSAs, the "urban" population that shall represent this variable is that of the county seat. The reason why a different variable is used for the data set for the consolidated SMSAs is because of the lack of data resulting from the small numbers of urban residents in the peri-urban areas of county seats. Furthermore, most of these county seats are small centers with probably limited differences in lifestyle and patterns of consumption, etc., from the population of the countryside proper. This limited variability is expected to be even more prominent in comparison of the population in the countryside to the urban population residing in the peri-urban areas of 
those county seats. Because the former have roots that extend quite deep into the area (both historically and psychologically), this may tend to reduce variations in the life styles and consumption habits insofar as it offsets the influence of urban dwellers in the peri-urban zone on the population of the county seats. This would reduce the chances of any one single group exerting impacts that would result in distinguishable changes in agricultural land use patterns in those peri-urban zones.

The interaction variable is expected to impact land use patterns in the peri-urban zones, including types of agricultural land use. The Department of Commerce (1978) states the case as follows:

A large and sometimes unrealized retail market potential exists in the non-SMSA areas of the nation. Business planners on an increasing scale are beginning to recognize and channel funds into these previously neglected areas. In addition to their market potential, non-SMSAs are repositories of labor, capital, and managerial expertise. Local banks are available to finance new construction, and manpower is abundant to fill job requirements. (p. 1)

(8) The Land-Use Regulation Variable. This variable (ZONING) is seen to act as a constraint against the conversion of peri-urban (agricultural) land to nonagricultural uses. This loss has been enormous, particularly in the U.S. over the past three decades (U.S. Department of Commerce, 1981). One major factor behind land conversion is the rise in the value of land per unit area that is brought about by land speculation and the nonagricultural uses of land. Healy and Rosenberg (1979) report that "in Delaware, announcement of the possible construction of a coastal oil refinery caused the value of nearby marshlands to rise from $\$ 40$ to $\$ 100$ per acre to $\$ 1,000$ per acre" (p. 227). It is obvious, then, that such rises in the price of land for nonagricultural uses negatively affects the long-term use of agricultural land, and, consequently, the kinds of crops to be cultivated (Coughlin, 1981).

One measure seems to slow, if not totally halt, the conversion of agricultural land to nonagricultural uses, and this is regulating the use of land for longer periods of time. Still, 
the results of such a process, that would be expressed as stable land uses, are expected to show up slowly since there are so many factors involved. These include farm profitability, urban growth pressures, land values, personal decisions, community expectations, taxes, and government programs, to name but a few. Coughlin (1981), in fact, warns of the enormous complexity of this issue.

Land use regulations are intended to controi the conversion of agricultural land to nonagricultural uses in areas threatened by urban takeover, which is particularly the case in peri-urban areas. Depending on the area in question, these regulations may range from mandatory measures, which are strict, comprehensive, and free of loopholes, to less stringent voluntary measures. Given the extent of the impact that such regulations might have on land speculation and land uses in general, and considering further that the variability in land use regulation reflects political boundaries, it is obvious that land use trends, including choices of crops as well as conversion of land to nonagricultural uses, would vary considerably from county to county or state to state to reflect the effects of these regulations. It is equally clear that the need for land use planning varies with the crop. Crops demanding more capital expenditure--e.g., greenhouse culture and vegetable production outside of greenhouses--will correlate more strongly with sites with long-term planning.

It should be realized, however, that unless there is a clear urgency for land preservation in a specific area, regulations are usually of a voluntary nature (Coughlin, 1981). The result of this is the so-called leap-frogging urbanization, which in the long run undermines the commitment to long-term planning for agricultural production. All of the United States' states have at least voluntary land use regulations; restrictive and mandatory land use regulations in the U.S. are still limited to a relatively small number of instances. Among these, however, the most popular are regulations that sustain agricultural zoning in 
peri-urban zones. Regulations of this kind, which involve both exclusive and nonexciusive zoning, have been applied so far to more than 270 localities in the U.S. (Coughlin, 1981).

The present study will evaluate the presence or lack of this kind of specific agricultural zoning regulation in peri-urban zones as a variable testing for the long-term effects of urban encroachment on crop selection, location of crops, and production intensity. These factors should correlate, since zoning regulations affect the availability of capital needed for crop production. For example, lands threatened with imminent urban encroachment would not tend to attract large agricultural investments; consequently, farmers would tend to choose crops that are short-term and less capital intensive--e.g., grains. On the other hand, perishables production, particularly greenhouse culture, would not tend to flourish in a climate of active land speculation, since its intensive production requires large fixed capital investments.

Following is a list of variables used in the present study, with a record of data sources (See Figure 9, below). 


\begin{tabular}{|c|c|c|c|c|}
\hline \multicolumn{2}{|c|}{ Yariable and Unit of Analysis } & Data Source & Xear & Table \\
\hline \multicolumn{5}{|c|}{ Dependent Variables } \\
\hline 1. WHEAT & Acres per county & 1 & 1982 & $\mathrm{XV}$ \\
\hline 2. VEGET & Acres per county & 1 & 1982 & XXVII \\
\hline 3. GREENH & Number of farms per county & 1 & 1982 & $\mathrm{XXX}$ \\
\hline \multicolumn{5}{|c|}{ Independent Variables } \\
\hline 1a. URBAN & Population of SMSA & 2,3 & 1980 & a \\
\hline \multirow[t]{2}{*}{ 1b. INTERACT } & Pop. of suburban area outside SMSA & 2,3 & 1982 & II, III, V \\
\hline & Pop. of county seat & 2 & 1980 & $b$ \\
\hline 2. LOTSIZE & Average size of farm/county level & 1 & 1982 & I \\
\hline 3a. GROWTH & Days per year over $32^{\circ} \mathrm{F}$ & 4 & 1981 & c \\
\hline 3b. PRECIPIT & Average inches per year & 4,5 & 1981 & c \\
\hline \multirow[t]{2}{*}{ 4. IRRIGTATE } & Wheat acreage & 1 & 1982 & $\mathrm{XV}$ \\
\hline & Vegetable acreage & 1 & 1982 & $\mathrm{XXVII}$ \\
\hline 5. FERTILIZ & Acreage under fertilization & 1 & 1982 & VII \\
\hline \multirow[t]{2}{*}{ 6.ZONING } & County/SMSA & 6 & 1981 & $c$ \\
\hline & & & 1979 & c \\
\hline 7. VALACRE & Value in dollars per acre in county & 1 & 1982 & I \\
\hline 8. ACCESS & Number of interstate lines in county & 7 & 1976 & c \\
\hline
\end{tabular}

Data Sources:

1. Census of Agriculare (AC82-A)

Tables: Supplementary Info:

2. Rand McNally Commercial Atlas, 1986.

3. U.S. Department of Commerce, 1985.

4. NOAA: Climate of the United States.

5. NOAA: Weather of U.S. Cities.

6. Coughlin, R. (1981), and Healy, R. (1979).

7. U.S. Dept. of Transportation

a. Rand McNally

b. Basic business data, by county

c. Study area data

Figure 9. Summary of variables and data sources. 


\section{EQUATIONS FOR TESTING THE HYPOTHESES}

In each of the four sets of data, three equations are employed for testing the research hypotheses--one for testing each dependent variable's correlation with the relevant independent variables. As listed earlier, the three dependent variables are acreage wheat harvest (WHEAT), the acreage vegetable harvest (VEGET), and the number of farms with greenhouse culture and nursery production (GREENH). Each of these three variables are represented in each of the four data sets.

Since the U.S. wheat region represents the pool or "universe" of cases for empirical testing in this study, the spatial distribution of data sets to be included for testing is necessarily located within this same wheat region. However, the major criterion for differentiating one data set from another is the physical location of that data set relative to the proximate SMSA market center (which is represented by the SMSA), since each data set (hence, each case in that set) is located an equal number of county units from the proximate market. Moreover, each county in every set is subjected to urban impacts similar to all other counties in that same set, be it from the county seat or the suburban population of the SMSA. This is seen to expose each unit of analysis (county) in every tier to urbanization impacts similar to those experienced by all other units of analysis in the same tier. These urbanization-related impacts constitute only one category in a longer list of variables that are hypothesized to influence the kinds and production intensity patterns of crops cultivated in the areas under study.

Following is the equation to be applied to the third and outermost tier of counties relative to the SMSA for testing the spatial distribution of harvested wheat acreage (WHEAT): 


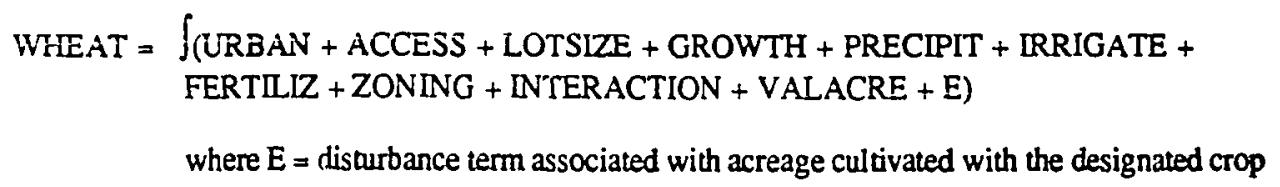

Similar equations are used for testing the location of harvested vegetable acreage as well as the number of farms cultivated with greenhouse culture and nursery production in the same (third) tier of counties, except that, of course, the irrigation variable is absent from the third equation, which is that for greenhouse culture, and the fertilizer variable is absent from both the second and third equations. The significance of irrigation and fertilizer variables on crop location relates to the fact that both are proven techniques for increasing productivity, which is essential for agriculture located at sites with higher land rent.

Following are the equations for perishable vegetable crops (VEGET) and greenhouse culture and nursery production (GREENH):

$$
\begin{aligned}
\text { VEGET }= & \int(\text { URBAN + ACCESS + LOTSIZE + GROWTH + PRECIPIT + IRRIGATE + } \\
& \text { ZONING + INTERACTION + VALACRE + E }) \\
\text { GREENH = } & \int(\text { URBAN + ACCESS + LOTSIZE + GROWTH + PRECIPIT + ZONING + } \\
\text { DITERACTION + VALACRE + E }) &
\end{aligned}
$$

Identical equations are to be used for testing in the second tier of counties, as well as for the tier of metropolitan counties. As for the consolidated SMSAs, the interaction variable will be represented by the suburban population living outside the main urban center of the SMSA. However, this variable is represented in the other tiers by the county seat population.

\section{IMPACTS OF THE MARKET FACTOR ON THE CULTIVATION OF THE WHEAT CROP}

Unlike perishables production, which is intended primarily for domestic consumption (Martin, 1987), grain production, both for silage and for food, is primarily intended for export to international markets (Ikerd, 1985). This factor ties market prices 
for grains in part to buyer conditions overseas and beyond the scope of overwhelming domestic influence. Ikerd (1985) reports that U.S. wheat export for the years 1979-81 amounted to 64 percent of domestic production for those years, whereas com exports totaled 30 percent of the same period's production. When added to this is the fact that export grain sales are not stable, the enormous difficulty of production forecasts and domestic control over demand become apparent. Frequently geopolitical and other nonmarket factors influence the international demand for U.S. wheat as much as conventional market factors, as is often the case with Soviet purchases of U.S. exports (The Utah Farmer-Stockman, Jan. 15, 1987). Among these factors would be periodic fluctuations in the state of export-market economies, and accidents of the kind that occurred at the Chemobyl nuclear power plant in the Soviet Ukraine in 1986. The same source cites other factors that would cause the Soviets to buy less American grain, among them being "the strong American dollar--relative to the Argentine peso and the Canadian dollar-aggressive marketing of major grain competitors, and Soviet problems in earning hard currency for more farm imports" (p. 13). Of course, this is only a small portion of the complexities of international grain marketing, for the Soviet Union is only one among many customers for American grain, and Canada and Argentina are not the only competitors of U.S. grain farmers. Further complicating the situation is the buying power of Third World countries, not to mention government programs for protecting local producers/exporters.

The domestic wheat market is not much easier to define or predict than is the international market. This is so for many reasons. First, the term "wheat" itself is generic, denoting five main classes of wheat that vary in end use, price per unit product, and the location of productions (see Figure 7). For this reason the trade of different varieties of wheat occurs between different regions of the U.S., including states and localities that 
produce wheat for export. This factor makes it quite possible having a population center listed as a wheat importer while at the same time the same population center is listed as a wheat exporter. Furthermore, it is difficult to predict the sources of these imports, and it is equally difficult to estimate future needs of wheat by-products, since such predictions must take into account such subjective factors as tastes, eating habits, etc., which are in a constant state of flux. Regardless of this, however, it is still expected that the variables FERTLIZE, IRRIGATE, and ACCESS will influence the locations for the produciton of wheat relative to the market. Specifically, the location of wheat production is expected to show a measureable correlation with these variables. 
CHAPTER V

\section{RESEARCH FINDINGS}

\section{INTRODUCTION}

As described earlier, the field area is composed of the fifteen states that make up the so-called "wheat region" of the United States (see Figure 7). The unit of analysis is the county. The research is designed so that three consecutive tiers of counties may be examined. The first of these tiers consists of the SMSA counties--that is, the metropolitan counties in the wheat region. The second and third tiers are the next two concentric bands of counties surrounding the SMSAs (see Figure 8). Data for the first tier of counties are presented in two forms: (a) an aggregated form that addresses each SMSA as a consolidated entity, and (b) a more detailed and detached form that addresses the SMSAs as disaggregated into individual counties.

This spatial structure was designed to test for the impact of urbanization on agricultural land uses at different locations around the urban market center, with space/distance acting as an umbrella under which those impacts $\propto c c u r$. At the same time, it is expected to test for the impact of other variables that may affect the cultivation of crops at different locations in peri-urban zones, particularly variables pertaining to natural environmental factors and technological development. In other words, the design of the present research is intended to provide equal opportunities for urbanization factors and environmental factors to function independently of one another insofar as they impact agriculture in the peri-urban zones, while at the same time being free to compete against one another. 
The present research design will also permit other variables, for example the effects of modern transportation, zoning regulations, lot size, and others, to influence the outcome of the present research so that their effects on agriculture may be measured, both in conjunction with and regardless of the impacts of the metropolis.

\section{PRELIMINARY ANALYSIS OF DATA}

The data exhibit both spatial and quantitative characteristics that appear highly supportive of the hypotheses advocated in this study, including the proposed sectoral cropping pattern of peri-urban agriculture. The preliminary analysis of data reveals the following characteristics:

(1) The spatial spread of the data strongly supports the research hypotheses that are summarized in Figure 5 and Figure 6, which indicate a rather concentric layout of agricultural land uses in the peri-urban zone (see Table II). This finding differs with most theoretical assertions, which suggest a zonal layout of crops in the vicinity of the market center, with crops limited to specific locations around the market center and no mix of crops in assigned locations. Data collected for the present research, however, indicates that this is not necessarily the case. Data show that the production of each crop, whether grains, vegetables, or greenhouse culture, tends to $\propto c c u r$ throughout the peri-urban zone, both very close to the market center and quite far away from it.

(2) Data show further that gradual changes occur in the acreage cultivated with the crops under study here as one moves from locations close to the market center toward locations more distant from it. This gradual change runs in two opposing directions. On the one hand, in the direction of the market center, there is a reduction in the number of acres cultivated in wheat. At the same time, moving away from the market center, one finds a gradual decline in acreage cultivated with vegetable crops as well as the artount of 
greenhouse culture and nursery production (see Table II). This would be even moreso the case when numbers are weighted so that different tiers have the same number of county units, as Table IV projects.

TABLE II

DISTRIBUTION OF AGRICULTURAL PRODUCTION IN PERI-URBAN ZONES, BY CROP AND COUNTY TIER

\begin{tabular}{|l|c|c|c|c|}
\cline { 2 - 5 } \multicolumn{1}{c|}{} & $\begin{array}{c}\text { Wheat } \\
\text { (in acres) }\end{array}$ & $\begin{array}{c}\text { Vegetables } \\
\text { (in acres) }\end{array}$ & $\begin{array}{c}\text { Greenhouse } \\
\text { (num. of farms) }\end{array}$ & $\begin{array}{c}\text { Total Num. } \\
\text { of Cases }\end{array}$ \\
\hline $\begin{array}{c}\text { Tier I } \\
\text { (SMSAs) }\end{array}$ & $5,803,787$ & 339,500 & 5,703 & 253 \\
\hline Tier II & $17,347,700$ & 404,565 & 2,357 & 467 \\
\hline Tier III & $14,184,713$ & 138,326 & 642 & 291 \\
\hline
\end{tabular}

Table II shows acreages cultivated in wheat in each of the three tiers of counties making up the study area. It also shows acreage cultivated in vegetables as well as the number of greenhouse and nursery products farms in these same areas. Finally, Table II shows the total number of instances (counties) located in each of the three tiers of counties. It should be noted that Tier I covers the SMSAs in the wheat region, while at the same time covering the metropolitan counties, since all counties considered SMSA counties are also considered to be metropolitan.

There is unquestionably more acreage cultivated in wheat in tiers two and three than in Tier I. Furthermore, the wheat crop in the peri-urban zones is distributed spatially in a manner that is opposite to the spatial distribution of the vegetable crop and greenhouse cultivation in the same areas.

Table III shows these same data expressed as percentages. It is important to note 
that the number of counties located in each of the three tiers is not equal; instead, more counties are located in tier two than in either of the other two tiers. In fact, there are almost twice the number of counties in tier two than in tiers one and three combined. However, after taking these size differences of the three tiers into account, the adjusted total acreage/number of farms cultivating each crop in each tier of counties continues to exhibit a gradual and orderly progression toward and away from the market center (see Table IV).

TABLE III

\section{DISTRIBUTION OF AGRICULTURAL PRODUCTION IN PERI-URBAN ZONES, BY CROP AND LOCATION, EXPRESSED AS PERCENTAGE OF ABSOLUTE NUMBERS}

\begin{tabular}{|l|c|c|c|}
\cline { 2 - 4 } \multicolumn{1}{c|}{} & Wheat & Vegetables & Greenhouse \\
\hline Tier I & 15.5 & 38.5 & 65.5 \\
\hline Tier II & 46.5 & 45.8 & 27.1 \\
\hline Tier III & 38.0 & 15.7 & 7.4 \\
\hline Total & 100 & 100 & 100 \\
\hline
\end{tabular}

Table IV is an adjusted/weighted comparison of crop distribution in the three tiers of counties. Data were adjusted to a normalized standard so that all tiers of counties would have relatively equally weighted numbers of cases. Accordingly, Table IV shows that the acreage cultivated with wheat increases systematically as one moves away from the market center, whereas acreage under vegetable cultivation and the number of greenhouse farms increase systematically in the opposite direction, that is, in the direction of the market center. These findings are consistent with the research hypotheses, which are summarized 
in Figure 5 and Figure 6.

TABLE IV

\section{DISTRIBUTION OF AGRICULTURAL PRCDUCTION IN PERI.URBAN ZONES, BY CROP AND LOCATION, EXPRESSED AS PERCENTAGE \\ OF ADJUSTED TOTAL FOR ALL TIERS}

\begin{tabular}{|l|c|l|l|}
\cline { 2 - 4 } \multicolumn{1}{c|}{} & Wheat & Vegetables & Greenhouse \\
\hline Tier I & 20.9 & 49.8 & 75.4 \\
\hline Tier II & 34.1 & 32.3 & 17.0 \\
\hline Tier II & 45.0 & 17.9 & 7.6 \\
\hline Total & $100 \%$ & $100 \%$ & $100 \%$ \\
\hline
\end{tabular}

In absolute terms there is more wheat acreage cultivated in Tier I--that is, the metropolitan tier of counties--than there are vegetables cultivated in all three tiers of counties combined. Nonetheless, there is more vegetable cultivation in absolute terms in the near proximity of the metropolitan market, where rent is high per unit land area, than in either of the two outer tiers of counties. This is so despite the fact that outer locations enjoy much lower rents per unit area and at the same time enjoy easy transport access to the metropolitan market at a reasonable cost. Figure 10, below, presents the data in tables III and IV comparatively in graphic form. 


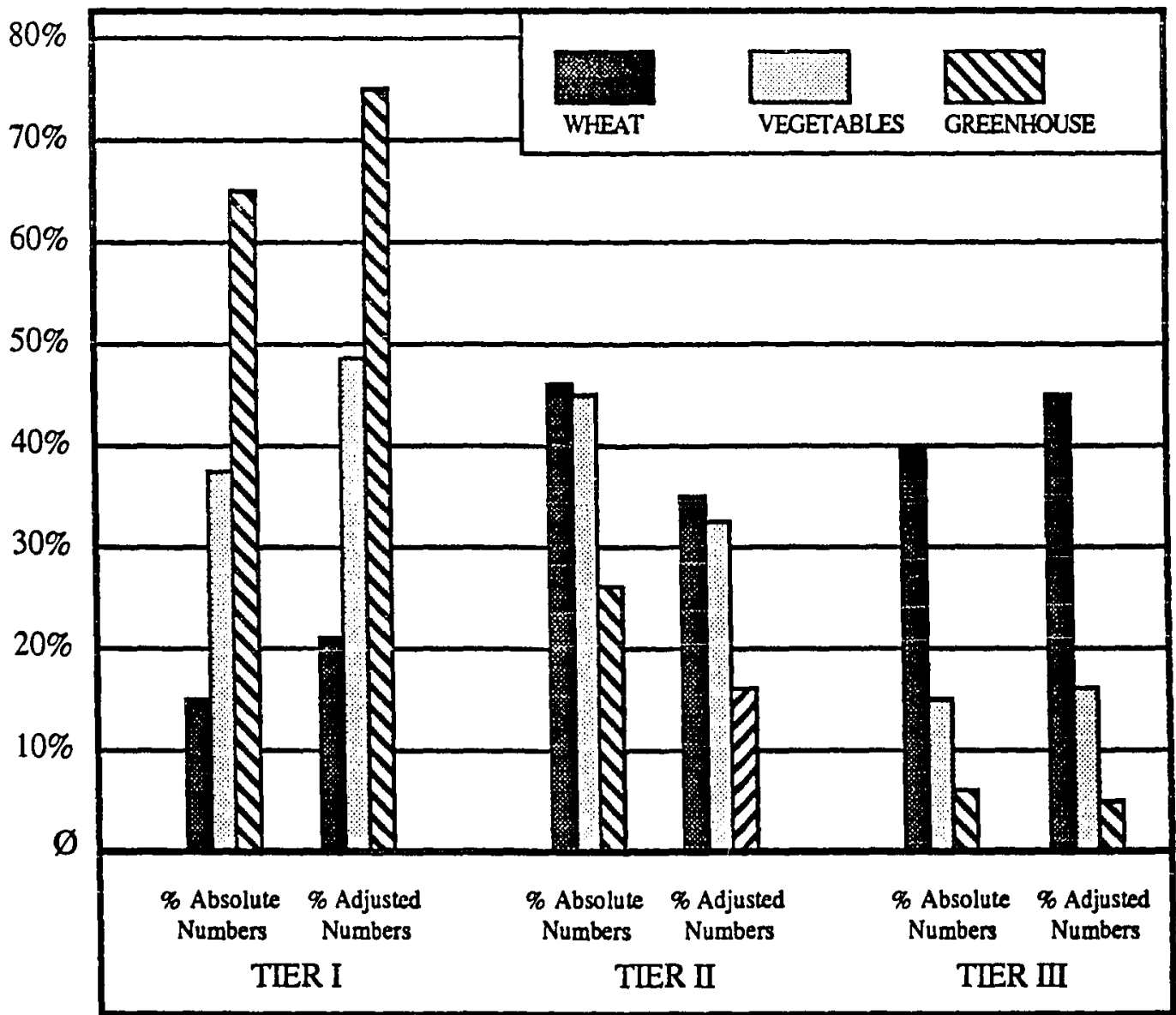

Figure 10. Graphic representation of crop distribution data as presented in Tables III and IV.

\section{GUIDELINES FOR DATA INTERPRETATION}

Having noted the spatial distribution, the question becomes, which factors are most responsible for the above-noted pattem of crops in peri-urban zones, and how important is each in relation to the other? To address this, one must ask the extent to which the present research hypotheses provide satisfactory explanations of these relations and their relative importance. What follows, then, is a description of the strategy employed here to extract the most comprehensive answers to these questions. 


\section{ANALYSIS OF THE REGRESSION OUTPUT}

The regression performed on the data is single-equation, least-squares regression analysis which is intended as an empirical check on the validity of the research hypotheses. The method of analysis is that of "backward elimination." The advantages of this method over others is that it allows for all of the predictors in the equation to be processed. This allows each of the predictors to associate with and influence the criterion-dependent variable; this, in tum, provides each with the opportunity to explain why, relative to it, each crop locates close to or distant from the market center.

Introducing all of the predictors into the analysis is particularly important to this research, largely because each was chosen in accordance with established theory and on the basis of an extensive and thorough review of all pertinent literature. For these reasons, backward elimination is the preferred method of analysis, since other stepwise routines like "forward selection" permit variables into the analysis only if they satisfy specific criteria of entry. The criteria of entry are established a priori and have nothing inherently to do with the specific differences between one set of data and another. Consequently, some "evidence" is invariably excluded from the analysis, thereby precluding its opportunity to influence the final outcome of the analysis. Furthermore, although the quantitative impacts of the excluded predictors might be limited, it is nonetheless true that other theoretical and/or qualitative impacts might ultimately prove to be quite significant. For this reason any method which limits the scope of the analysis is to be avoided.

\section{PHASES OF DATA ANALYSIS}

Data analysis is designed to occur on two phases. This procedure will best serve the goals of the present research. 


\section{Phase One}

Phase One is designed to evaluate the capabilities of the model as whole--all sets of predictors collectively--insofar as they explain the criterion variables (i.e., harvested wheat acreage, harvested vegetable acreage, etc.) and their locations relative to the market center. Stated differently, Phase One is designed to point out the proportion of $y$, the criterion variable, which is explained by each $x_{j}$, that is, each set of predictors. Therefore, this part of the analysis will be capable of measuring the degree of the variability of $\$ that may be explained by the relevant equation. Phase two, which is dealt with below, will explain the effect of each one of the predictors individually on the variability of $y$, the criterion variable.

The product best suited for describing the findings of Phase One of the analysis-that is, to quantify the sum of the impacts of the predictors on the criterion variable--is the multiple coefficient of determination, the so-called $R^{2}$. The $R^{2}$ is a measurement of the variability in $\chi$ (the criterion variable) insofar as affected by each $\underline{x}_{j}$ (each set of predictors) (Lewis-Beck, 1980:52). This is expressed another way by Heady (1971):

Agricultural economists have a tendency to put more emphasis upon the goodness of fit [a high $\mathrm{R}^{2}$ ] than is usually the case in engineering and other experimental sciences, rather than focusing diagnosis on the relative roles of the various exogenous factors associated with supply. (p. 249)

Nie, et al. (1975) value $\mathrm{R}^{2}$ as an adequate tool for describing the strength of the relationship between the sum of independent variables and the criterion variable. In the present study, the $\mathrm{R}^{2}$ will be reported for each tier of counties in order to account for the explanatory power of the predictors taken collectively. This result should prove valuable for pointing out the significance of specific locations on the cultivation of given crops in the peri-urban zone.

Obviously, higher $\mathrm{R}^{2}$ values are more desirable than lower ones, since they are indicative of a greater level of explanatory power in the predictors. Still, though, achieving 
a higher $\mathrm{R}^{2}$ is not an end in itself, since adding more predictors to the regression equation should raise the value of $R^{2}$. However, unless there is a clear and solid theoretical foundation for doing so, the resulting $\mathrm{R}^{2}$, although higher in absolute terms, will be difficult to defend or justify, since, as explained by Lewis Beck (1980), "tiny coefficients can be statistically significant" (p. 36).

Other products that are supportive of the $\mathrm{R}^{2}$ and which enhance its interpretability include the F-value associated with the regression output. In actuality, the F-value is an estimator of the linearity of the relationship--that is, the quality of the regression line. According to Heady and Dillon (1961), the F-value provides an overall test of the significance of the regression equation, since it in fact represents the ratio of the regression variance to the error variance. As such, a relatively large F-value is preferable to a smaller one. An F-value of 2.00 or more is considered statistically significant.

The Durbin-Watson index is also indicative of the quality of the relationships among the predictors. This index measures the presence or absence of autocorrelation problems among the residuals of regression and the dependent variable; should such difficulties exist, the addition or subtraction of variables from the equation and analysis might be indicated. However, only Durbin-Watson values less than 1.00 would cause concern about the existence of such problems.

Other products to be reported include sample size and the standard error of the regression. Both are helpful in the reproduction of additional statistics and are useful for explaining in greater detail the outcome of the analysis. In fact, the value of both the $\mathrm{R}^{\mathbf{2}}$ and the standard error of regression are inversely related, meaning that reporting both shows the direction and value of the overall correlation between the criterion variable and each set of predictors. Standard error is considered to be a "summary" statistic for the spread of the residuals around the "line of best fit." In other words, its value expresses the 
variables' degree of variance from a perfect linear association.

Also included in Phase One is the analysis of a residual plot, since it demonstrates visually the linearity of the relationships expressed in the equation. The object of this is determining randomly distributed residuals rather than residuals with specific patterns. This will indicate the existence of problems in the equation which may need special attention. The correlation matrix will also be checked for high collinearity among the equation's predictors. These regression products will be treated in the Statistical Package for the Social Sciences $\left(\right.$ SPSS $_{\mathbf{X}}$ ), which is that used in conducting the present analysis.

Findings of this first phase of the analysis are expected to show the degree of variability in the criterion variable which may be explained by the selected sets of predictors. Still, however, the practical value of these findings will remain limited, since the role that each predictor is to play in the final outcome is not yet clear.

\section{Phase Two}

Phase Two of the analysis is more detailed than Phase One, since it involves each predictor individually insofar as it associates with the criterion variable. The goal of Phase Two is to measure the impact of each predictor on the location of specific crops in the vicinity of the market center. The product best suited to this portion of the analysis is the regression coefficient, or $\underline{b}$.

Although $\underline{b}$ is a measurement of the level of association between each predictor and the criterion variable, it is considered sensitive to violations of homogeneity of variance assumption of multiple linear regression. Accordingly, the standardized B--the BETA-which adjusts for heterogeneity, is reported here. BETA represents associations of standardized units of measurement instead of raw data, which is the case for the $b$ (Heady and Dillon, 1961).

The partial correlation exnressed in this product describes associations between 
each independent variable and the dependent variable (the criterion variable), but with each independent variable's impact held constant. The control, here, is statistical and not literal. Moreover, the withheld variance is assumed to be linear throughout the equation, and in fact it is this linearity which makes partial correlation possible (Nie, 1976:302). These coefficients take either a plus or a minus sign, depending on the direction of the relationship; predictors with the highest coefficients are considered to have the strongest correlation with the criterion variable.

Many scholars prefer to report both the $\underline{b}$ and the standardized BETA. They also suggest otherwise reporting the standard deviation of all variables involved so that the reader can derive one set of coefficients from another (Pedhazur, 1982:250). Pedhazur recommends further that the researcher state clearly the role of theory in his or her report of an independent variable's impact that is derived from the degree of association between BETA and that variable.

In the present research, only the standardized BETA will be used in reporting on Phase Two analysis. This will help overcome many of the problems that the heterogeneity of the present data could very well cause for the $\mathrm{b}$ product. The decision to use the BETA instead of the b preserves the quality of the association between the two variables--i.e., the plus and minus signs. Moreover, the statistical significance reported in SPSSx, the reference used for this analysis, is statistically significant for the BETA, even though it was originally calculated for the raw $b$.

Such a method for handling the analysis means that the regression output will be presented in two distinct and yet highly interrelated parts. The first of these parts will provide information about the explanatory power of the applied model as a whole for predicting the patterning of crops in the peri-urban zone. The second part will provide more detailed information about the role that each predictor plays in influencing such 
agricultural land-use patterns in these peri-urban zones.

Both sets of regression output will be obtained for each of the three crops as occurring in each tier of counties under study in the present research.

\section{THE EMPIRICAL TESTING OF THE CULTIVATION OF THE WHEAT CROP}

Phase One: The Sum of the Explanatory Power of the Regression Model for Wheat Harvested in the Peri-Urban Zone

Checking for linearity in the regression equation and for multicollinearity among the predictors are two essential precautionary preliminaries. A check of residuals for the harvested wheat acreage shows a fairly random distribution, with no distinctive patterns. However, the correlations matrix showed a multicollinearity problem of a magnitude of .85 between the predictors URBAN and INTERACT, which represent urbanization.

This level of multicollinearity is a problem which needs to be addressed. One method of handling it would be to drop one of the collinear pair from the analysis; this solution, however, should take into account both theory and statistical significance. The predictor retained should be the one most closely associated with the analysis, or else have the larger coefficient.

Since both predictors in the present study were chosen based on theory, both were checked for statistical significance by dropping each one temporarily from the analysis. This allowed determination of the one most closely associated with the criterion variable, so that it could be retained. In the present study the retained predictor is URBAN. Dropping it from the analysis resulted in a much lower $\mathrm{R}^{2}$ than did dropping INTERACT. Furthermore, the values of the coefficients associated with URBAN are more consistent with the research hypotheses.

The correlation matrix also shows the predictors PRECIPIT, GROWTH, 
VALACRE, and LOTSIZE all to be collinear, although at a low level ranging between 4 and .5. Literature holds that this is a tolerable level of collinearity and that it does not require action of the kind taken with the multicollinear pair described above. As such, it was decided that all these collinear predictors would be retained. More encouraging in this regard is that dropping them from the analysis one at a time, as was done with the troublesome predictors URBAN and INTERACT, did not significantly alter the analysis. One reason for this is the huge net of interrelationships among variables in multivariate analysis, which discourages specificity in the relationships. Notwithstanding, these collinears need to be watched closely, and their outcomes should be considered with these collinears in mind.

Table V lists the products of Phase One of the analysis-that is, it lists in tabular form the overall impacts of the set of predictors on the criterion variable. 
TABLE $V$

REGRESSION OUTPUT FOR THE ACREAGE OF HARVESTED

WHEAT AT THREE LOCATIONS IN THE PERI-URBAN ZONE

\begin{tabular}{|c|c|c|c|c|c|}
\hline Product & $\mathrm{R}^{2}$ & F-Value & $\begin{array}{l}\text { Durbin- } \\
\text { Watson }\end{array}$ & $\begin{array}{l}\text { Standard } \\
\text { Enror }\end{array}$ & $\begin{array}{l}\text { Sample } \\
\text { Size (n) }\end{array}$ \\
\hline SMSA & .55 & $13^{* * *}$ & 1.5 & 7074 & 106 \\
\hline $\begin{array}{c}\text { Tier I } \\
\text { (metro) }\end{array}$ & .41 & $19^{* * *}$ & 1.7 & 2367 & 253 \\
\hline Tier II & .61 & $76^{* * *}$ & 1.5 & 2039 & 467 \\
\hline Tier III & .45 & $26^{* * *}$ & 1.6 & 3838 & 291 \\
\hline
\end{tabular}

*** Statistically significant at .001

A quick glance at Table $V$ shows the set of predictors to explain a relatively large proportion of the variability in the criterion variable WHEAT (harvested wheat acreage) for all tiers of counties. Table $V$ explains variability that reaches levels of up to 55 percent for wheat harvested in the consolidated SMSA counties; up to 41 percent for wheat harvested in Tier I counties; up to 61 percent for wheat harvested in Tier II counties; and up to 45 percent in Tier III counties. The table shows these ratios as $R^{2}$ values.

These empirical findings demonstrate, among other things, that wheat cultivation is not foreign to the metropolitan tier of counties, as the theoretical literature on this issue frequently presumes. This is obvious in the $\mathrm{R}^{2}$ values for this tier of counties, which reaches a level high enough to be situated between the two values describing the two most 
outer tiers of counties. Moreover, the F-values associated with these findings are relatively high, ranging between 13 and 76 . High F-values are indicative of greater variance emerging from the regression equation relative to the error variance. In other words, it is another indication of a greater linear association with the criterion variable. All F-values show a quite high level of statistical significance--above the .001 level. Table $\mathrm{V}$ also shows relatively high Durbin-Watson values, all of them exceeding 1.00. This shows no autocorrelation problems.

\section{Phase Two: The Role of the Individual Predictors in the Location of Wheat Cultivation in} the Peri-Urban Zone

The regression results reported in Table $\mathrm{V}$ are supported by the preliminary analysis reported earlier in this chapter. The regression results also show clearly that the harvested wheat acreage extends into different locations in the peri-urban zone, including sites in the near vicinity of the market center. Both of these findings are of limited applicability, however, since they leave unanswered the crucial questions pertaining to the specific roles of each predictor in influencing the outcome.

This lack is satisfied, though, with the partial correlation coefficient in standardized form--the BETA--since it shows the degree of association between each predictor and the criterion variable. Because of the standardization, comparisons are permitted between as well as within groups which are otherwise indivisible, because, among other things, the BETA is not sensitive to heterogeneity of variance nor to changes of measurement units in the data. This is achieved without jeopardizing the statistical significance of the findings, since the BETA reflects the statistical significance as much as does the b.

Table VI tabulates the values for the BETA (coefficient) associated with each predictor in the regression equation as applied to harvested wheat in different locations in the peri-urban zone. The larger the coefficient, the stronger the association between the 
pairs (Norusis, 1983). Frequently greater levels of statistical significance are associated with larger BETAs.

TABLE VI

BETA VALUES OF REGRESSION OUTPUT FOR ACREAGE OF WHEAT

HARVESTED AT THREE LOCATIONS IN THE PERI-URBAN ZONE

\begin{tabular}{|l|c|c|c|c|}
\cline { 2 - 5 } \multicolumn{1}{|c|}{ Location } & $\begin{array}{c}\text { SMSA } \\
\text { COUNTIES }\end{array}$ & $\begin{array}{c}\text { TIER I } \\
\text { (Metro.) }\end{array}$ & TIER II & TIER III \\
\hline Uariable & $-.168^{*}$ & .049 & $-.068^{*}$ & .044 \\
\hline VALACRE & -.090 & $-.164^{* * *}$ & $-.330^{* * *}$ & $-.347^{* * *}$ \\
\hline IRRIGATE & $.168^{*}$ & $.122^{* *}$ & $.129^{* * *}$ & $.095^{*}$ \\
\hline PRECIPIT & .021 & $-.092^{*}$ & -.017 & .010 \\
\hline GROWTH & -.010 & .006 & .031 & $-.159^{* * *}$ \\
\hline FERTILIZ & $.698^{* * *}$ & $.506^{* * *}$ & $.721^{* * *}$ & $.575^{* * *}$ \\
\hline LOTSIZE & -.097 & .082 & -.005 & .010 \\
\hline ACCESS & -.071 & -.025 & .005 & -.003 \\
\hline ZONING & -.051 & -.004 & $.088^{* * *}$ & -.022 \\
\hline
\end{tabular}

* Statistical significance at level of .1 or better.

** Statistical significance at level of .01 or better.

*** Statistical significance at level of .001 or better. 
This table presents a great deal of information. Among other things, it shows that in the Tier I counties the predictor FERTILIZ leads all others with its extremely large BETA; it is followed by the predictors VALACRE and IRRIGATE. The values for these three, all of which are statistically significant at .01 or better, are $.51,-.16$, and .12 , respectively; these figures are indicative of an association between these predictors and the criterion variable, harvested wheat acreage. In the SMSA counties these same predictors led the others with their high coefficients and statistical significance.

Such a high level of association between these predictors and the criterion variable should not be surprising, though; VALACRE reflects cultivation's location in metropolitan areas, and IRRIGATE and FERTILIZ reflect the need for higher intensity production in these areas where farmers must compete with characteristically nonagricultural land uses. Such an interpretation of these findings is supported by the large and statistically significant coefficient with minus sign associated with the predictor VALACRE, indicative of a reverse relationship between higher land value per acre and the cultivation of wheat in the metropolitan environment. Such a reverse association with VALACRE makes it in effect necessary that production taking place on those sites be implemented at higher intensity levels, even when such higher intensity raises production costs per unit product. It is worth noting that a plus sign accompanies the coefficient for the predictor FERTILIZ at all production locations considered in this study. Such an understanding of the relationships is supported by the large and statistically significant coefficient with plus sign associated with the predictor IRRIGATE, since this, too, is indicative of a strong forward association between this predictor and the cultivation of wheat in the metropolitan region. It is understandable that increased irrigation means higher intensity production per unit area. It also means higher costs per unit product. Moreover, the large and statistically significant coefficient with minus sign $(-.17)$ associated with the predictor URBAN also supports the 
interpretations thus far, since urbanization is assumed to discourage the use of land for activities that are not urban in character. It should be mentioned that the predictor URBAN is assumed to function as a proxy variable on behalf of urbanization. The large coefficient with minus sign for the predictor URBAN could be held responsible more than any others in the equation for the large coefficient with plus sign associated with the predictor FERTILIZ, since the minus sign is indicative of the lack of compatibility of the land uses in question. The result of this is that these uses either get rejected at these sites, or in an attempt to remain are forced to produce at higher intensity levels.

These findings for SMSA counties are repeated in the data for Tier I counties, since there, too, the predictor FERTILIZ has the largest coefficient, again followed by VALACRE and IRRIGATE. The signs for these three predictors in Tier I duplicate those experienced in the SMSA counties, including the fact that both are statistically significant at .01 or better.

In Tier II one finds the coefficients for the predictors FERTILIZ, VALACRE and IRRIGATE still leading the others. Not surprisingly, the same remains the case in Tier III. As important as this, however, is that the signs associated with the coefficients again duplicate those found in the preceding tiers of counties. This should indicate a powerful unifying influence which affects wheat cultivation in the wheat region, one which goes beyond the impact of location relative to the nearest market center. Note that most of the wheat produced in these tiers of counties ends up in the international market. Note, too, that the term "wheat" covers a multitude of classes of grain which vary considerably in both characteristics and end use. Furthermore, wheat goes through several intermediate processes before it is fit for human consumption; these intermediary production processes generally take place in locations other than those of cultivation. Given these conditions, the linkage with local markets is likely to be very thin. 


\section{THE ENVIRONMENTAL ARGUMENT}

One significant factor affecting the location of wheat production is the impact of the natural environment. It was hypothesized that natural environmental factors significantly influence the production locations of wheat. The two variables selected to represent the environment's influence are PRECIPIT and GROWTH--the average annual rainfall and the number of days of the year with temperatures above zero, respectively.

A look at these predictors as they appear in Table VI shows quite modest values for the coefficients for both at most locations. In addition, the coefficients carry minus signs in most cases. This indicates an inverse relationship with the criterion variable. These values are a bit surprising in light of the hypothesis. However, there are several interpretations of such an outcome.

One is that it pays cultivating wheat in peri-urban areas at locations with high rent per unit area as long as production is highly intensive. This could be so because wheat is not particularly sensitive to specific environmental requirements. Instead, wheat can survive fairly well in diverse environmental conditions and still provide a satisfactory yield (Arunachalam, 1965; Laut, 1965). The large coefficients we saw for FERTILIZ and IRRIGATE appear to reinforce this interpretation. Furthermore, a large share of production ends up on the international market, which may reduce the impact of local variations on production locations. However, this explanation conflicts with theoretical assertions which contend that the crop/fallow cycle is the most appropriate intensity measure for wheat produced in the peri-urban zone, and not the added-inputs factor.

Another explanation for tr: unsatisfactory coefficients for the environmental variables might pertain to the extremely broad array of environmental conditions in the wheat region in the United States. This could be creating the low levels of association, 
since environmental diversity is met with the production of five major classes of wheat, each of which varies significantly from the others in their environmental requirements.

Another possible explanation for the small value of the environmental coefficients and the inverse coefficients is the relatively high collinearity between the environmental and other variables in the equation. It is known that collinearity can not only reduce the value of coefficients associated with the collinear pairs, but can also turn its sign to a minus one. However, testing this last possibility by alternately dropping both collinear pairs from the analysis did not result in any measurable differences from the already stated values.

To rectify this discrepancy, the wheat region was divided into homogeneous subregions in order to focus the measure:nents. This was done by dividing the region along lines identical to those presented in Figure 7, which represents the four areas or subregions of production for the various classes of wheat (see Table VI, below). Regressions for the four subregions resulted in more statistically significant findings than were achieved in the regression for the wheat region as a whole. 
TADLE VT

BETA VALUES FOR THE VARIABLES PRECIPIT AND GROWTH FOR
THE CRITERION VARIABLE WHEAT PRODUCTION
IN FOUR ENVIRONMENTAL SUBREGIONS

\begin{tabular}{|c|c|c|c|c|}
\hline Subregion & Variable & $\begin{array}{c}\text { Tier I (metro } \\
\text { counties) }\end{array}$ & Tier II & Tier III \\
\hline \multirow{2}{*}{$\begin{array}{l}\text { Texas- } \\
\text { Nebraska }\end{array}$} & precipitation & $.08^{* *}$ & .01 & .09 \\
\hline & growth & -.29 & $-.21^{*}$ & -.09 \\
\hline \multirow{2}{*}{$\begin{array}{l}\text { South Dakota- } \\
\text { Minnesola }\end{array}$} & precipitation & .03 & $-.56^{* * *}$ & $-.50^{* *}$ \\
\hline & growth & -.16 & -.03 & -.02 \\
\hline \multirow{2}{*}{$\begin{array}{c}\text { Missouri- } \\
\text { Ohio }\end{array}$} & precipitation & -.10 & $-.42^{* * *}$ & .20 \\
\hline & growth & .03 & $.18^{*}$ & $.54^{*}$ \\
\hline \multirow{2}{*}{$\begin{array}{l}\text { Idaho- } \\
\text { Oregon }\end{array}$} & precipitation & -.14 & .04 & .04 \\
\hline & growth & .17 & -.03 & -.04 \\
\hline
\end{tabular}

* Statistical significance at level of .1

** Statistical significance at level of .01

*** Statistical significance at level of .001

The regression output reported in Table VII reveals several statistically significant products, although most show an inverse relationship with the criterion variable, wheat production. This is particularly true for the variable PRECIPIT in most subregions, and particularly at outer locations. This is clearly seen in the northern subregions, where temperatures are relatively mild and hence the effectiveness of precipitation greater due to reduced loss to evaporation. Another explanation for this outcome is that a good deal of wheat is dependent on irrigation.

Even more telling in Table VII is the comparison of the two sets of findings--those for PRECIPIT in the southern subregions incorporating Texas, Oklahoma, Kansas and Nebraska, and those for GROWTH in the northern subregions incorporating Missouri, Indiana, Illinois, and Ohio. Values for both variables in both subregions relate positively with wheat production in both subregions. Although the statistical significance of these 
findings varies with distance from the nearest market center, it is noteworthy that precipitation correlates positively and in a statistically significant manner with wheat production in relatively hot regions, where the length of the growing season is not as critical to the success of the crop as is the availability of moisture. This interpretation of the results is supported by the positive and statistically significant BETA for the variable GROWTH in the northem subregions, where the length of the growing season is quite important for winter wheat. The Missouri-Ohio subregion is the only one of the northern subregions that grows winter wheat, which requires $40-42$ weeks to mature, whereas spring wheat takes only 16-18 weeks to mature. These findings parallel the views of Schlebecker (1973) in recognizing the selective impacts of natural environmental factors on crop location.

Note that the correlations matrix for the subregions shows collinearity above .3 between the predictors IRRIGATE and PRECIPIT, which could account for the reduced statistical significance for at least one of them. Furthermore, IRRIGATE should substitute for PRECIPIT in most cases where land rent is high and/or production is meant for trade.

\section{OTHER VARIABLES IN THE EQUATION}

Other predictors in Table VI include the variable ACCESS. The BETA coefficients for this variable at different locations are small and are not statistically significant at any location. This is indicative of their limited effect on the criterion variable. What is more significant with these coefficients, however, is the consistency of the minus sign associated with the coefficient for nearly all tiers of counties (see Table VI). This indicates a reverse association between the pair of variables. One interpretation of these findings is that a large portion of the wheat produced in this region ends up in either the international market or large metropolises distributed around the U.S. In fact, this might increase the significance 
of quality and price of transportation in the location and subsequent marketing of wheat.

Next is the predictor ZONING, which also shows low coefficients with a minus sign, and which also lacks statistical significance except for Tier II counties. This is again indicative of a reduced though reverse relationship with wheat production at most locations. Such low values could have been the result of dummying the variable, since doing this reduces the range of values to be analyzed as well as contributing occasionally to a minus sign in a coefficient. In fact, dummying might be the major contributor to the small coefficient associated with the predictor ACCESS, too, since it also is represented by discrete values. Still, these minus values for the predictor ZONING support earlier findings about the predictors VALACRE and URBAN and their respective minus signs.

Finally is the predictor LOTSIZE, whose coefficients are not statistically significant at any location. Moreover, the BETA values for this variable show an inverse relationship with wheat production in the peri-urban zone, which supports what has already been found about the predictors VALACRE, URBAN, and ZONING--that is, the inverse association with wheat production at locations close to the market center.

\section{THE EMPIRICAL TESTING OF THE CULTIVATION OF THE VEGETABLE CROP}

The reporting of the findings for the empirical test of the cultivation of vegetables in peri-urban zones will also proceed in two phases, as was the case for wheat. In the first phase we shall focus on evalıating the overall explanatory power of the regression equation as it pertains to factors affecting the cultivation of vegetables at different locations in the peri-urban area. In phase two, we shall focus on the role of each predictor individually. Each phase will have its own set of products, as they are extensions of the products used in the analysis of the previous crop. 
Two products need checking for assumption violations before proceeding with the analysis. One is the matter of residuals, and the other is the correlations matrix. Checking the residuals shows no systematic patterns. It also shows no serious deviations from normality. However, there is a slight tilt to the left near the baseline of the curve. One way of handling a more serious tilt in this direction is taking the square root of the data. However, the present tilt is seen to be quite mild, so that no action was deemed necessary.

The correlations matrix shows multicollinearity between the predictors URBAN and INTERACT at a magnitude of .83 . Both are considered proxy variables of urbanization's impact on agricultural land uses in the peri-urban zone, so this level of correlation is unacceptable. It was decided, therefore, that one of the two making up the multicollinear pair should be dropped from the analysis. Altemately dropping each of these predictors from the equations indicated retaining the predictor INTERACT in the analysis, and disposing of URBAN. This conclusion was based on the former's proving to be more significant statistically. Accordingly, it showed a higher $R^{2}$ value. It should be noted that in the case of wheat cultivation, a similar problem was experienced. In that case, however, the predictor URBAN was retained since it showed a higher level of statistical significance than did the predictor INTERACT. It might be remembered that the predictor URBAN represents the urban population of the SMSAs proper, while the predictor INTERACT represents suburban population of the SMSA, and/or the population of county seats in outer tiers of counties.

Further inspection of the correlations matrix shows the predictor PRECIPIT as being collinear with the predictors LOTSIZE and VALACRE in all tiers of counties. It also shows predictors LOTSIZE and VALACRE to be collinear. The level of these correlations ranged from .42 to .52 . Such levels of collinearity are considered tolerable by most analysts. Still, and as a precautionary step, these collinear pairs were tested by alternately 
dropping them from the equation to test their effects on the findings. This test did not result in any measurable differences from the values already achieved, and it was therefore decided that the analysis would proceed with all mildly collinear pairs retained. However, more attention will be paid to these variables in the analysis.

Phase One: The Sum Explanatory Power of the Regression Model for the Location of Vegetable Production in the Peri-Urban Zone

This portion of the analysis shall deal with the overall explanatory power of the regression model for the cultivation of perishable crops in peri-urban areas. The products most appropriate to this portion of the analysis are the $R^{2}$, the $F$-value, and the DurbinWatson index. The values for these products are listed in Table VIII.

Table VIII is quite similar to Table V, except that the former does not include the variable FERTILIZ. The reason for the absence of this important variable is the lack of data regarding it as pertains to vegetable cultivation. 
TABIE YIII

\section{REGRESSION OUTPUT FOR THE ACREAGE OF HARVESTED VEGETABLES AT THREE LOCATIONS IN THE PERI-URBAN ZONE}

\begin{tabular}{|c|c|c|c|c|c|}
\cline { 2 - 6 } Location & $\mathrm{R}^{2}$ & F-Value & $\begin{array}{c}\text { Durbin- } \\
\text { Watson }\end{array}$ & $\begin{array}{c}\text { Standard } \\
\text { Eror }\end{array}$ & $\begin{array}{r}\text { Sample } \\
\text { Size (n) }\end{array}$ \\
\hline SMSA & .844 & $.66^{* * *}$ & 1.4 & 366.1 & 106 \\
\hline $\begin{array}{c}\text { Tier I } \\
\text { (metro) }\end{array}$ & .977 & $1304^{* * *}$ & 2.1 & 55.5 & 253 \\
\hline Tier II & .624 & $94^{* * *}$ & 2.1 & 93.0 & 467 \\
\hline Tier III & .430 & $26^{* * *}$ & 2.1 & 86.0 & 291 \\
\hline
\end{tabular}

*** Statistically significant at .001

Table VIII reveals several important things. First, we see that the collective explanatory power of the present model for vegetable cultivation at different locations in the peri-urban area appears quite high. This is clear in the high $R^{2}$ values, which range between .97 and .42 . Among other things, this means that a large proportion of the variability in the criterion variable ( $(x)$ is being explained by the set of predictors. The statistics mean that up to 97 percent of the factors affecting vegetable production in the metropolitan tier of counties are explained by the model.

However, the gradient for the $\mathrm{R}^{2}$ drops between the market center and the outermost ier of counties; if anything, this change of values in the $\mathrm{R}^{2}$ means that the factors affecting the production of these crops are not the same at all locations. Instead, 
they vary with location and distance from the market center. This finding supports the research hypothesis, since it was hypothesized that vegetable production in the immediate vicinity of the market would reflect conditions that are specific to those locations. Such an interpretation agrees with the figures reported in Table VIII, which show the $\mathrm{R}^{2}$ to be higher in metropolitan counties than in SMSAs.

Still, the declining values for the $\mathrm{R}^{2}$ in the direction away from the market center do not lead to a vanishing of those values in the outer tier of counties. And this is indicative of remaining viability in these outer locations to this kind of perishables production. Such a finding, however, contradicts conventional theory on zones of production of perishable crops, which limits production of these crops to the inner areas of peri-urban zones.

At the same time, these findings do not contradict the research hypothesis, which emphasizes the significance of the metropolitan regions in the cultivation of perishables, since the success of inner locations in perishables production, according to the hypothesis, is not conditioned on failure of the outer locations. Instead, it is based both on the individual qualities of those crops and on the ability of inner regions to support this kind of land use. It should be noted, though, that much of the literature on this issue acknowledges the two-location initiative for perishable crop production, although there is not enough specificity about many aspects of this finding. In addition, the vast majority of the supporting literature gives the impression that it is just a matter of time before the production of these crops is totally eliminated from the immediate vicinity of the urban market, to the eventual advantage of the outer locations. Most experts cite the truck as the bigges influence of such an outcome.

Table VIII also shows large F-values, ranging from 1304 to 26 , all of them significant at better than .001 level. Also significant are figures for the Durbin-Watson index, all of which exceed the level of 1.00. This is indicative of the absence of 
measurable autocorrelation problems among the set of predictors. And finally, we see large sample sizes, which helps to overcome problems stemming from assumption violations, particularly those pertaining to normality of the distribution.

Phase Two: Role of the Individual Predictors in the Locating of Vegetable Production in the Peri-Urban Zone

In Phase Two, we shall examine the roles of each of the predictors individually as regards their effects on the spatial distribution of the vegetable crop in peri-urban areas. Here, too, the partial correlation coefficient in standardized form--the BETA--is used to accomplish this goal. Table IX lists the BETA coefficients for each predictor as it associates with each location. Following is an analysis of these correlations and a summary of their implications. 
TABLE IX

BETA VALUES OF REGRESSION OUTPUT FOR ACREAGE OF VEGETABLES HARVESTED AT THREE LOCATIONS IN THE PERI-URBAN ZONE

\begin{tabular}{|c|c|c|c|c|}
\hline Location & SMSAS & $\begin{array}{l}\text { TIER I } \\
\text { (Metro.) }\end{array}$ & TIER II & TIER III \\
\hline INTERACT & .097 & $.026^{* *}$ & $.086^{* * *}$ & $.111^{*}$ \\
\hline PRECIPIT & .077 & -.011 & $-.098 * *$ & $-.140^{*}$ \\
\hline GROWTH & -.012 & $-.016^{*}$ & -.007 & .002 \\
\hline ACCESS & $.059 *$ & $.014^{*}$ & $.053^{*}$ & -.011 \\
\hline VALACRE & -.022 & $.352^{* * *}$ & $.193^{* * *}$ & $.220^{* * *}$ \\
\hline LOTSIZE & .041 & .013 & .012 & -.04 \\
\hline ZONING & $.155^{* * *}$ & .009 & -.034 & -.028 \\
\hline IRRIGATE & $.900 * * *$ & $.986^{* * *}$ & $.753^{* * *}$ & $.596^{\neq \neq \neq}$ \\
\hline
\end{tabular}

* Statistical significance at level of .1

** Statistical significance at level of .01

*** Statistical significance at level of .001

Table IX shows the predictor IRRIGATE, which is statistically significant at .001 , leading all other predictors in Tier I, an indication of the large amount of variability in the criterion variable (acreage of harvested vegetable crop) which can be explained by this predictor. A second predictor with a relatively significant coefficient is VALACRE. These same two predictors lead all others in the outer two tiers (Tiers II and III) as well. Both had large coefficients in the previous analysis, that is, in explaining the locations of wheat 
cultivation.

Whereas a minus sign distinguishes the VALACRE coefficient in the analysis of wheat production, for vegetable production the indication is reversed; that is, VALACRE shows a plus coefficient, which is indicative of a straightforward relationship between VALACRE and vegetable production. This is quite a significant development; a preliminary explanation of this relationship might go as follows:

(1) Land value, whether rent per unit area or fair market value per acre, is a major factor affecting land uses and the decision-making process for location decisions of agricultural production in peri-urban areas.

(2) This change of sign in the vegetable crop might indicate basic differences between wheat production and vegetable production with regard to higher land values per unit area, or with regard to urbanization impacts on land uses in the peri-urban zone.

(3) At the same time, both crops seem to efficiently apply the added-inputs approach as a means of combating higher land rent per unit area in the direction of the market, be they irrigation, fertilizer, or both. What matters most is that the added-inputs method is a certified means of creating higher intensity production per unit area and, consequently, higher revenues per acre of land.

Other predictors in Table IX include INTERACT, which also is statistically significant at all locations and has a relatively large coefficient and a plus sign for all tiers of counties. This indicates a strong positive association with vegetable crop location in periurban areas. The findings are statistically significant for all tiers of counties. Tying this to earlier findings about predictors VALACRE and IRRIGATE, one finds that these three predictors carry values and signs that support one another. It also contradicts findings for similar predictors in the analysis of wheat production. When taking into account the minus sign for the predictors URBAN and VALACRE in the analysis of wheat production, 
differences between the two categories of crops with respect to the impacts of urbanization and/or higher land rents per unit area become apparent, even though both crops have managed to survive in the near vicinity of the urban market. Remember, too, that both URBAN and INTERACT are proxies for urbanization. Moreover, a strong case could easily be made about a strong relationship between the variable VALACRE and the impacts of urbanization on land values.

The coefficients for the predictor INTERACT at different locations reveal a number of interesting things. First, the BETAs for this variable are more statistically significant in metropolitan counties than in SMSAs, a clear indication of the greater impact of the local market on location decisions than distant markets. This is supported by statistically significant BETA values for the relationship between local markets and outer locations having urban nonmetropolitan population centers. The implication here is that current literature might be understating the impact of urban nonmetropolitan centers on agricultural land uses in their vicinity while overstating the impact of metropolitan areas on crop location at outer locations. In fact, the low values, lacking statistical significance, for the predictor URBAN seem to discredit the importance of metropolitan centers in this regard. Because of these uncertainties, in Table X, below, the variable INTERACT was dropped from the analysis in order to test this metropolitan impact as reflected in the variable URBAN. The result was lower values and a statistically insignificant BETA. The run also resulted in lower $\mathrm{R}^{2}$ values. 
TABLE X

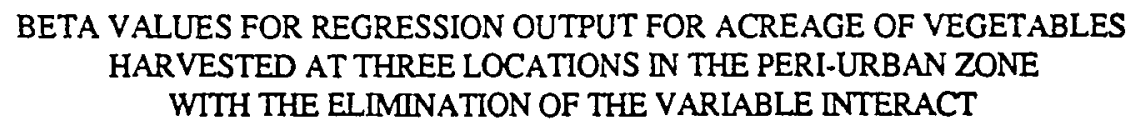

\begin{tabular}{|c|c|c|c|c|}
\hline Location & SMSAs & $\begin{array}{c}\text { TIER I } \\
\text { (Metro.) }\end{array}$ & TIER II & TIER III \\
\hline URBAN & $.073^{*}$ & -.003 & .008 & .035 \\
\hline PRECIPIT & .076 & -.012 & $-.092 *$ & $-.126^{*}$ \\
\hline GROWTH & $-.018^{*}$ & -.016 & -.001 & .022 \\
\hline ACCESS & .056 & .012 & $.051^{*}$ & -.017 \\
\hline VALACRE & $-.010^{* * *}$ & .046 & $.208 * * *$ & $.240^{* * *}$ \\
\hline LOTSIZE & .043 & .012 & .006 & .069 \\
\hline ZONING & .161 & .008 & -.028 & -.028 \\
\hline IRRIGATE & $.902^{* * *}$ & .984 & $.747^{* * *}$ & $.589 \div \neq \neq$ \\
\hline $\mathrm{R}^{2}$ & .840 & .976 & .617 & .420 \\
\hline
\end{tabular}

* Statistical significance at level of .1

* Statistical significance at level of .01

*** Statistical significance at level of .001

The next predictors to consider are the environmental variables PRECIPIT and GROWTH. Both show minus coefficients, indicating a reverse relationship with the criterion variable, and both are statistically insignificant, particularly in the metropolitan counties. This outcome parallels earlier findings about the positive and strong association between urbanization and vegetable production that was demonstrated in the predictors 
IRRIGATE, VALACRE, and INTERACT. At the same time these findings agree with the hypothesis, which asserted that urbanization and characteristics of the crop itself assert priority over environmental factors as determinants of the location of vegetable production.

Next is the matter of the gradient of the coefficients, and one must ask, How systematic are the changes in the values of these coefficients at different distances from the market center? In other words, How does location relative to the market affect the impact of different predictors on the criterion variable?

The coefficient for the predictor IRRIGATE drops regularly as one moves farther from the market center; however, it retains its plus sign and statistical significance throughout all tiers of counties. This finding is quite significant, both for the large coefficient characterizing this predictor and for its plus sign. Having these values increase most in the metropolitan counties (Tier I) indicates that the most intensified production takes place at these inner locations. By the same token, it indicates the readiness of these crops to lend themselves to cultivation at such intensified levels.

The gradual rise of production intensity as one moves toward the market center--as seen in the IRRIGATE coefficient--shows this higher intensity per unit area facilitating agricultural land uses at locations close to the market center which otherwise would be limited to nonagricultural land uses of a characteristically urban nature. This trend is quite clear in the case of wheat production as well.

As for the predictors INTERACT, VALACRE, PRECIPIT, LOTSIZE, ACCESS, ZONING, and GROWTH, all show systematic gradation of the value of the coefficient as one proceeds along the line between the market center and the outermost locations. For example, the coefficient for VALACRE decreases in the direction away from the market center, although its sign remains positive and statistically significant. Among other things, this might indicate the close association between vegetable production and higher land rents 
resulting from urbanization. This finding is supported by the change of values associated with the predictor LOTSIZE, which shows a larger coefficient in metropolitan counties and a smaller one farther out. This indicates the ability of vegetable cultivation to thrive on smaller size lots, which are characteristically a by-product of urbanization.

The predictor ZONING also exhibits a larger coefficient in the direction of the market center, indicating compatibility between agricultural land use and urbanization. This is reinforced by the ACCESS variable, whose coefficient also increases in the direction of the market center. The negative sign and low value of the coefficient for this variable in Tier III counties might indicate basic differences in the needs of cultivators of these crops in outer locations compared to those situated in inner locations, which is supportive of another facet of the research hypothesis. For example, it is possible that frequency, variation, and easy access to different modes of transportation could be considered satisfactory to producers in the market vicinity. At the same time, outer producers might be more interested in having access to specific modes of transportation. The BETA values for ZONING increase dramatically in the tier of SMSA counties, indicating a very strong relationship between this zoning regulation and crop location at the level of SMSA counties. The sharp fall at the next tier reinforces the particularly urban nature of the impact of this variable.

\section{THE EMPIRICAL TESTING OF THE PRODUCTION OF GREENHOUSE CULTURE AND NURSERY PRODUCTION IN PERI-URBAN ZONES}

The final production category to be analyzed includes greenhouse culture (GC) and nursery products (NP). Along lines similar to those for the earlier analyses, the present investigation will be executed in two successive phases. The first phase will report the sum impact of the collective set of predictors on the criterion variable; that is, it will measure the 
capabilities of the regression equation to explain the variability in $y$, the criterion variable at different locations relative to the market center. Phase two will discuss the role of each predictor individually in explaining locational variability in $\mathrm{y}$. Both phases, though, are seen to complement rather than supplement one another, because knowing how much of the variability in $y$ can be ascribed to each predictor in the set does not result in knowing the predictive capabilities of the model as a whole, and vice versa. This is even moreso the case because of the interaction that commonly takes place between predictors executed together (Kerby, 1970).

Phase One: The Sum Explanatory Power of the Regression Model for the Locating of Greenhouse Culture and Nursery Production at Different Locations in the Peri-Urban Zone

Table XI presents the regression products for the sum impact of the factors hypothesized to be most responsible for the location of greenhouse culture (GC) and nursery production (NP) in peri-urban zones. 
TABLE XI
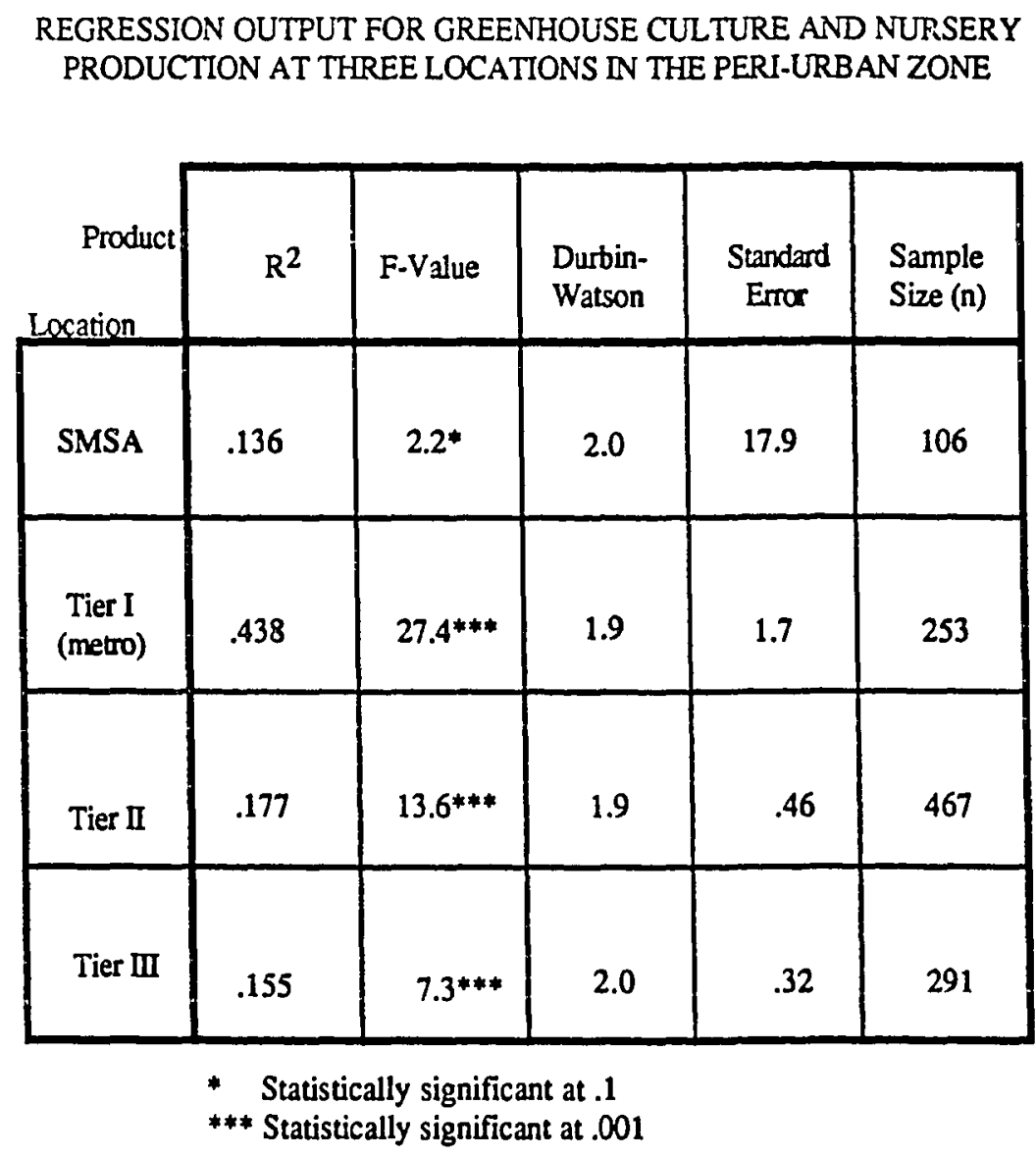

We see in Table XI $\mathrm{R}^{2}$ values gradually decreasing as they progress away from the metropolitan center. This means that the present model is more expressive of production conditions of $\mathrm{GC}$ in the near vicinity of the market than farther away. It also means that factors of production for these crops vary with location, in a way that is similar to the variance seen with vegetable production. It also means that the present set of predictors, which were hypothesized to describe more prominently GC production in peri-urban areas, are satisfactory, especially in Tier I counties, where they claim up to 44 percent of the effect in the variability in the criterion variable. Still, comparing these $\mathrm{R}^{2}$ values with those seen in the analyses of wheat and vegetable production shows measurable discrepancies in the 
explanatory power of the present model. The most likely reason for these discrepancies goes back to the absence of specific predictors from this latest equation which address GC and NP, particularly those which in the earlier analyses explained the locations of wheat and vegetable cultivation--that is, FERTILIZ and IRRIGATE. These predictors are not included because of a lack of data. In fact, data on fertilizer is lacking even for vegetable production. This is so despite the fact that vegetable production is known for its intensive use of fertilizers to achieve essential high levels of productivity.

There is great diversity among crops included under the headings greenhouse culture and nursery products. At one extreme are crops cultivated in fully controlled environments; at the other extreme are crops that are almost totally dependent on the natural environment. Measurable differences in durability $: j$ environmental condition, market price, and marketing techniques, among other things, exist between various crops in this category of analysis. These differences, then, show up as discrepancies in location and methods of cultivation that in turn affect their coverage by a limited number of variables. Despite these discrepancies and the heterogeneity which distinguishes this category of crops, the $\mathrm{R}^{2}$ values resulting from the regression seem to support both the hypothesis and the theoretical literature, which describes cultivation of these crops as more metropolitan and less rural. This is clear in the large $\mathrm{R}^{2}$ for Tier I counties.

Other products in Table XI that help to describe the explanatory power of the regression equation include the F-values, which are significant to .001 in three out of four tiers of counties; the F-value for the SMSA tier of counties was significant at .04. The Durbin Watson index exceeds 1.00 in all tiers of counties, indicating the absence of serious autocorrelation problems in this set of data. 
Phase Two: The Role of Individual Predictors in Locating Greenhouse Culture and Nursery Production In the Peri-Urban Zone

This second phase of the analysis involves pointing out the role of individual predictors in the locating of GC and NP in the vicinity of the market center. Table XII provides the BETA ccefficients for the predictors thought to be most influential on the location of GC and NP in peri-urban areas.

TABLE XII

BETA VALUES OF REGRESSION OUTPUT FOR GREENHOUS CULTURE AND NURSERY PRODUCTION AT THREE LOCATIONS

IN THE PERI-URBAN ZONE

\begin{tabular}{|l|l|l|l|l|}
\cline { 2 - 5 } \multicolumn{1}{l|}{} & $\begin{array}{c}\text { SMSA } \\
\text { COUNTIES }\end{array}$ & $\begin{array}{c}\text { TIER I } \\
\text { (Metro.) }\end{array}$ & TIER II & TIER III \\
\hline Variable & $.200^{*}$ & $.087^{*}$ & $.108^{*}$ & $.247^{* * *}$ \\
\hline URBAN & .035 & .009 & $-.206^{* * *}$ & $-.114^{*}$ \\
\hline PRECIPIT & .023 & $.227^{* *}$ & $.189^{* * *}$ & $.072^{*}$ \\
\hline GROWTH & $.169^{*}$ & $.086^{*}$ & $.126^{* *}$ & -.040 \\
\hline ACCESS & .054 & $.438^{* * *}$ & $.308^{* * *}$ & $.238^{* * *}$ \\
\hline VALACRE &. .015 & -.011 &. $.086^{*}$ &. .042 \\
\hline LOTSIZE & .063 & $.254^{* * *}$ & $.099^{*}$ & $.153^{* *}$ \\
\hline ZONING & &
\end{tabular}

* Statistical significance at level of .1

** Statistical significance at level of .01

*** Statistical significance at level of .001 
All predictors in Table XII show significance, whether statistical, theoretical, or both. Further, although the role of the predictor with the largest coefficient varies with location and with distance from the market center, the table still shows systematic change in coefficient values between the market center and more distant sites for the majority of predictors in this set. Most telling of these relationships include the following:

(1) The predictors VALACRE and URBAN have large coefficients and plus signs throughout the tiers, indicative of a straightforward positive relationship between urbanization and both GC and NP.

(2) Findings pertaining to urbanization-related predictors, namely URBAN and VALACRE, do not disagree with values associated with the environmental variables PRECIPIT and GROWTH. The entire group does not seem to be in conflict with the research hypothesis. For example, PRECIPIT's coefficient is low and lacks statistical significance in both SMSA and Tier I counties, an indication of a low association between annual precipitation in this area with both GC and NP, as would be expected with mostly environmentally controlled agricultural production. Such an inverse association with PRECIPIT becomes even less significant in the outermost tiers of counties, where the coefficients' signs continue as minus, while the level of statistical significance increases. The other environmental predictor, GROWTH, however, responds differently. The coefficient for this predictor is statistically significant with larger values, particularly in the direction of the market center, an indication of a stronger association with GC and NP. There is no one simple interpretation of these results for the environmental variables. Instead, one or more of the following is likely.

(a) The reason the environmental variable PRECIPIT appears less significant is that irrigation substitutes for precipitation when possible. As already seen, irrigation associated quite significantly with both wheat and vegetable cultivation; hence, the only reason this 
variable is not included in the present portion of the analysis is the lack of data.

Regardless, this probability of substitution is encouraged by the fact that the rights to water for irrigation are easily acquired in the United States, and the water itself is inexpensive. Substitution becomes a different issue, though, when it comes to the variable GROWTH and the substitution of artificial light and heat for the sun's warmth and light, since such substitutions are significantly more costly. This would be the case even if these natural agents are able to penetrate transparent materials housing greenhouse culture. This means that technological limitations and high costs make it necessary for entrepreneurs to search their environment for alternatives that give more yield for less costs per unit of product.

(b) The second view is that the minus sign on the coefficient for the predictor PRECIPIT might not be authentic. It could have resulted from the collinearity problem between it and many others in the equation. If so, the original sign of the coefficient might in reality be plus, and both environmental predictors positively influencing the locating of GC and NP in the vicinity of the market center. To check this possibility, it is necessary to break the test region down into subregions, much as we did with wheat and vegetable cultivation. This will be done at the end of this section.

(c) Another way of looking at this issue is by evaluating GROWTH together with the predictor VALACRE, since both carry large and statistically significant coefficients which decrease as they move farther from the market center. Greenhouse culture is a land use with relatively high yield per unit area. This makes it rewarding cultivating these crops at sites with high rent per unit area. Such sites most often coincide with above average environmental conditions, since quality of the environment is one important criterion of assessing land value. These viewpoints agree with the fact that a large number of urban centers are located on the most productive and fertile soils of their respective regions.

(d) Another explanation may relate to production factors. GC and NP involve 
crops that are produced in totally controlled environments at high expense and are directed primarily at the metropolitan market. At the same time, many of these same crops are cultivated in the open at environmentally suitable locations and are marketed to neighboring consumers in smaller, nonmetropolitan centers. This locational division saves transportation costs, exploits differences in land rent and labor costs between smaller and larger centers.

(4) Among the remaining predictors, ZONING shows the highest statistical significance. Moreover, the coefficients for this predictor carry plus signs and rise in value in the direction of the market center, indicating a positive relationship between GC and this kind of land regulation. However, as Table XII shows, variations between tiers of counties are clear. The outcome here contrasts with findings for the same sets of data run for vegetable crop locations (see Table X). One interpretation of this may be that the impacts of zoning are more urban than metropolitan when it comes to the location of GC. In other words, GC generally occurs on a small scale, by individual farmers satisfying needs of nearby markets. Interpreting GC location as a function of production scale is supported by the positive and statistically significant BETAs for the variable ZONING in the outer tiers of counties (Table XII).

(5) ACCESS is also statistically significant, but moreso at locations closer to the market center, which is indicative of the significance of the distance to market in this kind of agricultural production. This supports both theory and the research hypothesis. However, the low value of the coefficient, with minus sign, which appears in the outermost tier of counties should be interpreted to more or less indicate the importance of quality over diversity of transportation to this kind of land use. This also might indicate the reduced significance of the central metropolitan market on crops that are cultivated in outer locations and are destined for neighboring nonmetropolitan markets. 
(6) Finally, there is the predictor LOTSIZE, whose coefficient is both low and minus for all tiers of counties. This outcome could be the result of collinearity with other predictors more than it is indicative of a reverse relationship between land values and the GC and NP production in peri-urban areas. In fact, the correlations matrix shows this variable to have a relatively high collinearity with a number of predictors.

The last step is to check the environmental diversity issue insofar as it may be causing the low value in the coefficients for the environmental variables PRECIPIT and GROWTH. As stated, doing so requires dividing the wheat region into homogeneous subregions, as was done for the analysis of wheat and vegetable cultivation. However, doing so did not bring about any significant change in the minus sign associated with the environmental variable PRECIPIT (see Table XIII), although it did result in a higher value for the coefficient.

TABLE XIII

BETA VALUES FOR THE VARIABLES PRECIPIT AND GROWTH FOR THE CRITERION VARIABLES GREENHOUSE CULTURE AND NURSERY PRODUCTION IN FOUR ENVIRONMENTAL SUBREGIONS

\begin{tabular}{|c|l|l|l|l|}
\hline Subregion & Variable & $\begin{array}{c}\text { Tier I (metro } \\
\text { counties) }\end{array}$ & Tier II & Tier III \\
\hline \multirow{2}{*}{$\begin{array}{c}\text { Texas- } \\
\text { Nebraska }\end{array}$} & precipitation & -.013 & -.133 & .122 \\
\hline & growth & $.196^{*}$ & $-.138^{*}$ & $.421^{* * *}$ \\
\hline $\begin{array}{c}\text { South Dakota- } \\
\text { Minnesota }\end{array}$ & precipitation & .436 & $.537^{*}$ & $.772^{* *}$ \\
\cline { 2 - 5 } & growth & -.424 & -.144 & -.234 \\
\hline $\begin{array}{c}\text { Missouri- } \\
\text { Ohio }\end{array}$ & precipitation & $-.257^{*}$ & -.109 & .113 \\
\cline { 2 - 5 } & growth & .015 & -.085 & .036 \\
\hline $\begin{array}{c}\text { Idaho- } \\
\text { Oregon }\end{array}$ & precipitation & -.033 & $-.618^{*}$ & -.636 \\
\cline { 2 - 5 } & growth & -.027 & .296 & .275 \\
\hline
\end{tabular}

* Statistical significance at level of .1

** Statistical significance at level of .01

*** Statistical significance at level ố .001 
Additionally, more positive relationships with relatively large BETAs are emerging for GROWTH for most locations. The only exceptions are for GROWTH in the TexasNebraska subregion, and for PRECIPIT in the South Dakota-Montana subregion. For both the outcome is positive and statistically significant for all but one location. Since long growing seasons are common in the south, however, and because the north has few problems with evaporation, the findings may in fact indicate that greenhouse culture and nursery products farmers may sometimes avoid environmental extremes and select environmental conditions similar to those for crops that are not grown under controlled environmental conditions. Such an outcome should be seen as confirming the earlier finding that there was little significance, if any, in precipitation over the location of GC and NP. The most likely explanation for this outcome is to be found in the strong reliance of these crops on irrigation. Also, these findings indicate the extended reliance on controlled environments in the production of these crops, which in tum supports the hypothesis of the present research. 


\section{CHAPTER VI}

\section{SUMMARY ANALYSIS AND CONCLUSIONS}

The last chapter stopped short of offering specific answers to some of the focal questions raised in the present research and was somewhat general in addressing the impacts of the factors that were hypothesized to affect the patterning of peri-urban agriculture. This matter is to be deait with in part one of the present chapter. The impacts of the predictors will be assessed as to the degree to which they affect the patterning of specific crops in the various tiers of counties. This grouping of the influencing factors will rely heavily on the information presented in Tables VI and XII. The second portion of this chapter will present specific conclusions derived from this research. Additionally, problems encountered in the research and analysis will be discussed and suggestions for future research will be outlined.

\section{PART ONE: SUMMARY ANALYSIS}

\section{The Accessibility Variable}

The variable for accessibility--ACCESS--measures the sum impact of modes of transportation on the location of agricultural production in peri-urban zones. It does not

directly measure the effect of linear distance to market, although the design of the research itself makes this possible.

Beginning with the harvested wheat crop, we see in Table VI a low coefficient for the variable ACCESS, with minus sign and no statistical significance in all tiors of counties. This indicates a limited impact of the diversity of transportation on production 
locations for wheat, particularly at locations in the immediate vicinity of the uroan market. In other words, a diversity of modes of available transportation does not affect the location of wheat production.

This finding may have resulted from one of the following two considerations, or from both together. First, the kind rather than the mere diversity of transportation may be the true determinor of the location of wheat production. A review of the literature shows explicitly that in the case of wheat, unit trains take the lead over all other modes for transport within the U.S., whereas the ocean-going steamers prevail for overseas shipments. The second possible explanation pertains to the fact that the central market adjacent to production zones may not be the primary consumer of the vast majority of that area's production. The produce is instead destined for a broad array of national and international markets. This finding supports the research hypothesis, as will be discussed below.

Tuming to the vegetable crop, Table IX shows that the coefficient for the predictor ACCESS is large, positive, and statistically significant, especially at locations closest to the market center. This finding supports claims about the positive association between vegetable production and the urban market. This finding supports the research hypothesis, which predicted that easier access to the market, particularly due to shorter distances, would induce entrepreneurs to cultivate these sites, and that production would continue despite the adverse impact of higher rents per unit area at these locations.

Table XII shows that greenhouse culture and nursery production also associates strongly with ACCESS, again particularly at locations closer to the market center; the coefficient is high, positive, and statistically significant at those sites. This also indicates the significance of the varied modes of transportation in determining the location of these forms of agricultural production in peri-urban zones. The finding here is similar to that for the vegetable crop, and might also be explained by diversity of transportation. Equally 
important, this positive and statistically significant association between ACCESS and perishables production reinforces the sectoral nature of peri-urban agriculture. (This association holds for wheat production in the same areas, and it should be recalled that data shows a rather mixed production in the peri-urban zone, with wheat cultivation occurring alongside perishables production.) Still, the evidence for sectoral patterning is somewhat inconclusive, since due to the macro scale of the analysis it might still be argued that a concentric pattem holds true. It may be the case that the variable ACCESS accounts for apparent dissipation in one tier of counties.

One may finally conclude generally about the variable ACCESS in the following terms: Access to transportation varies with the type of crop requiring transport. Diversity has a dominating influence on the locations of the majority of perishable crops. However, such access does not seem to be important to the locating of grains. Moreover, the linear distance to the nearest market center, although important for some crops--particularly perishables and greenhouse culture--does not appear to significantly affect every crop. To reiterate, the variety of modes of available transportation is most important, offering significant "convenience" to the transfer of perishable crops to the urban market. This convenience, then, could translate as costs per unit product/mile, or more properly, as quick delivery, therefore enhancing the marketing edge. However, grain production seems to be more inclined toward quality transportation, where costs per mile are reduced by virtue of extended travel time. Still, with access to new technology, low-cost transport, etc., indirectly consumed grains uppear capable of occupying sites close to the market center, regardless of the higher land rent per unit area.

\section{Qther Predictors in the Equation}

Table VI shows that five predictors have relatively large coefficients and mixed signs, indicative of a high degree of association with the criterion variable--in this case 
harvested wheat. These five are FERTLIZ, IRRIGATE, VALACRE, URBAN, and LOTSIZE. The first two carry plus signs with their coefficients, which indicates a positive, straightforward association with the criterion variable. The last three carry a minus sign, indicative of a reverse association, that is, detachment from the criterion variable.

Even with these contradictory signs, these five predictors evidence no contradictory associations either with the criterion variable or with one another. The implication is that wheat production is not compatible with the urban environment. This is so because, among other things, it is not the kind of agricultural product that urban consumers would want or need fresh from the farm in the shortest possible time. Moreover, the crop itself is not particularly perishable, so that short distances to the neighboring market would provide no advantage. This is clear in the negative signs with the large coefficients for URBAN, VALACRE, and LOTSIZE, since all three reflect to varying degrees the impact of urbanization on land uses in the surrounding peri-urban area.

At the same time, however, the predictors FERTILIZ and IRRIGATE are positively associated with wheat production, and seem to be rectifying the effects of the aboveindicated inconveniences of urbanization. Both predictors are, in fact, intensity measurements, and as we have already seen, intensifying production is one important way of fighting the negative effects of higher land rents per unit area, which is another corollary of urbanization. Significant here is that the predictor FERTILIZ has the highest share of the $\mathrm{R}^{2}$ change for wheat in all tiers of counties. In a stepwise regression check the change in $R^{2}$ across Tiers I, II, and III amounted to $.32, .45$, and .30 percent out of $.42, .60$, and .45 percent respectively.

This excessively high share of the $\mathrm{R}^{2}$ for FERTILIZ leads to two conclusions. One is the commercial nature of wheat production, since fertilizer is an added input that raises production costs per unit product; the second is the high degree of resistance to this kind of 
land use exhibited by other land uses in peri-urban zones. This last is clear from the large coefficients with minus signs for the predictors VALACRE, URBAN, and LOTSIZE. This is something which the added-inputs method appears to be combatting with success, since such a large amount of cultivated wheat acreage is located so close to the market center.

Tuming to the vegetable crop, in Table IX we see that the predictors with large coefficients (and again with mixed signs) are IRRIGATE, VALACRE, INTERACT, LOTSIZE, and PRECIPIT. Note that there is no data for fertilization of the vegetable crops, so that this variable is not included in the list. The first four of the above five predictors carry a plus sign with their coefficients, indicating a positive association with vegetable production in peri-urban areas, particularly at locations close to the market center. As for PRECIPIT, it is an environmental variable and carries a minus sign for all tiers of counties. This is indicative of a diminished, if not reverse, association with vegetable production. However, this is understandable for commercial production where costs of production per unit area come next to yields because of the large share of fixed costs attached to production, which therefore encourages intensified production incentives. This view is supported by the low coefficient with minus sign for the predictor GROWTH, which is the other environmental variable in this set.

A stepwise regression check of the $\mathrm{R}^{2}$ for each predictor shows IRRIGATE leading all others, with values of $.79, .58$, and .37 percent out of $.84, .62$, and .43 percent for Tier I, II, and III counties respectively.

In Table XII, greenhouse culture and nursery production show large coefficients for all predictors except LOTSIZE. Furthermore, all of the remaining predictors except PRECIPIT are positively associated, although even this predictor has a large coefficient with minus sign, which increases as it moves away from the market center. There is little doubt that greenhouse culture is tied to urbanization. This is so because of the high market 
price and the need for freshness when marketing the commodity. More broadly, these findings on greenhouse culture and nursery production reflect a culmination of the impacts of package technology, which pits factors of production and distribution against one another. This leads to trade-offs and substitutions among the factors, which results in lower production costs and/or higher revenues. It also results in some crops occupying other than their original locations, or else extending farther out beyond their usual territory while still remaining commercially sound.

\section{The Impact of the Marketplace}

Several legitimate questions emerge from this discussion. One concerns the point at which one draws the line between the impacts of the local, national, and international markets on patterns of land uses in peri-urban areas, and whether or not a solid criterion even exists for this determination. The difficulties of providing definite answers for these points are illustrated by the following.

First, the system of land uses is not static. It changes constantly, so much so, in fact, that Grigg (1969) suggests that models dealing with agricultural production be designed with build-in change. Second, as Datoo (1978) suggests, the agricultural system is embedded in a hierarchy of systems whose influences can be explained as a series of constraints. But it cannot be eliminated completely. Third, there are always more factors involved in the production and spatial distribution of crops than one can possibly count. In fact, this led Phipps (1983) to conclude that part of the outcome of any analysis of land use patterns "may not be attributable to any known pattern" (p. 69).

As for the impact of the international market on the location of peri-urban agriculture, and consequently on the value of agricultural land in these areas, one should accept the fact that impacts of the international market are part of the overall impact of the so-called "situation," known to geographers. In essence, it is another facet of the impacts 
of the urban market on land values in peri-urban areas, for it is impossible to separate one from the other. This situation is complicated even further by the increased accessibility of farms to local and regional markets, which in a sense makes all farmland act as though it were peri-urban, or, the other way around, makes the peri-urban zone extend almost everywhere in the countryside (in developed countries). The following from Ikerd (1985) in this regard is pertinent:

Increased international trade likely has done more to change the economic environment of U.S. agriculture than has any other single development of the past 20 to 30 years. Food grain exports have almost tripled in the last 25 years. Feed grain exports have increased by four-and-one-half times . ... Growing exports fueled rapidly rising commodity prices and an economic boom in agriculture ... [generating] unrealistic expectations concerning future profitability of agriculture. Those optimistic expectations were reflected in a strong demand and rising prices of agricultural land. (p. 14)

However, VALACRE's impact on perishables production is much different from its impact on wheat production. Here VALACRE associated strongly and positively with both vegetable and greenhouse production, a clear sign of the compatibility between these crops and the peri-urban environment. Still, perishables are produced in these areas at high intensities, which is clear in the large BETA value with plus sign for the predictor IRRIGATE. As pointed out earlier, for lack of data no representative variable for fertilization has been included in this portion of the analysis. The same is the case for the predictors IRRIGATE and FERTILIZ for the analyses of greenhouse culture and nursery production. Otherwise, both variables would have shown closer associations with both crops. Heady and Dillon (1961) name the application of more fertilizer for the purpose of attaining higher yields per unit area as compliance with economic principles and the mixing of resources, since this results in the best uses of the land. Such a mixing of resources might include the substitution of fertilizer for land, particularly when the former is cheaper than the latter. This view is defended by Isard (1956). These are additional sources of confusion that militate against drawing clear lines around the sphere of influence of 
markets.

Still, the plus sign in the association of VALACRE with vegetable and greenhouse production indicates clearly the primary differences in the type of the relationship they enjoy with the peri-urban environment as opposed to that encountered by wheat production. Again, these varied associations seen in correlations with perishables versus grain production reflect trade-offs between a larger set of factors relating to package technology mentioned earlier. The outcome of these trade-offs, once again, is crops being allowed to easily change locations and still remain commercially viable. Factors contributing to this development include production intensity, urbanization, and characteristics of the market, to name just a few. This point will be highlighted in the following discussion about a second factor that should be accounted for when growing crops in the near vicinity of the market--urbanization.

\section{The Impact of Urbanization}

As noted earlier, the complexity of urbanization as a variable prevents any single index from being constructed to represent or measure its impact (Toboda, 1976). In the present study, urbanization is represented by two variables, INTERACT and URBAN (see Chapters IV and V for discussion). In fact, the complexity of this issue is clear in the results presented in Tables VI, IX, and XII, where the predictors URBAN and INTERACT associate differently with each crop, each one showing not only widely different values for the coefficients, but different signs as well. This wide variability means two things. First, urbanization is a force that should be reckoned with when cultivating crops in the peri-urban area; and, second, it is not a cliche that may be applied indiscriminately regardless of crop category or location relative to the market.

That this is true is clear in the sign and value of the coefficients for URBAN and INTERACT in their relationship with the crops under study. Whereas URBAN shows a 
strong and statistically significant reverse correlation with wheat production, both it and INTERACT correlate strongly and positively with both vegetable crops and greenhouse culture/nursery products. This is a clear indication of the significant variations between both crops in their relationships with urbanization. It should be noted that all of these crops compete for the same locations in the market vicinity.

\section{The Impact of the Environmental Factors}

The two environmental variables, PRECIPIT and GROWTH have low values and/or a minus signs for the coefficients at most locations for all three crops, which indicates a weak effect of these environmental factors on the locations of production for these three crops. The only exception to this was with the predictor GROWTH as it associated with greenhouse culture and nursery production; the correlation here was positive and statistically significant at all locations. Otherwise, both predictors are statistically insignificant in their effect on the location of vegetable and wheat production.

The correlation matrix shows collinearity ranging from .4 to .5 between these environmental variables, as well as with other predictors in the equations--particularly VALACRE and LOTSIZE. As mentioned earlier, collinearity reduces the value of the coefficients and/or turns their sign to minus. Having such collinearity makes one hesitate to accept as final these low and/or inverse associations. To rectify this situation, both the predictors VALACRE and LOTSIZE were alternately dropped from the analysis. However, doing so neither about a higher value of the coefficient nor changed its sign. The outcome, then, strengthens earlier findings suggesting the limited effectiveness of these environmental variables in influencing the locating of crops in the vicinity of the urban market, as long as the wheat region is treated as a single entity. It is becoming increasingly clear that collinearity is not what is holding these values to their low measured levels. However, when the wheat region is divided into homogeneous subregions, 
statistically significant findings were obtained for some subregions (see Table VII). Table VII shows that both variables PRECIPIT and GROWTH had large, positive, and statistically significant BETA values for some regions. Although this was shown to indicate the impact of environmental factors on wheat production, it was pointed out as well that they were overshadowed by the impact of economic location.

These new findings about the environmental variables support in principal the research hypothesis dealing with wheat production, as it was posited that whitai profuction in peri-urban areas would be encouraged by suitable environmental conditions, because, among other things, having such growth conditions would raise productivity fer unit area at no extra cost per unit product. Bear in mind that higher intensity also proved effective for encouraging the locating of wheat production around the market, contrary to conventional theory in this regard.

Regardless, there may still be "legitimate" factors that reduce the influence of these environmental factors on the location of wheat production. One such factor may relate to the fact that the wheat plant is quite resilient to a wide range of environmental conditions. This is reflected in the wide range of conditions under which the wheat crop is presently distributed, both inside and outside the research area. Arunachalam (1965) has pointed out that "successful plant breeding for centuries has resulted in various varieties of wheat fit for diverse regions. It spread to areas that were once deemed to have no agricultural potentialities" (p. 3). Views similar to these, and views going farther and attesting to the weakness of environmental factors as a deterrent against the spread of wheat cultivation, are presented by Thompson (1962). He, too, acknowledges the adaptability of wheat to "subhumid and semiarid regions where rainfall varies a great deal from year to year and site to site, and so does temperature" (p. 149)

Sir Josiah Stamp justifies these views about the spread of wheat to diverse locations by counting five other factors, besides the impact of the environment, which he sees 
influencing the patteming of crops in different areas. He calls them "principles," and they are as follows:

(1) The effect of weight or bulk of the crop per unit of value with regard to transport charges; (2) the need for diversification and seasonal requirements; (3) the need for diversification to maintain soil fertility; (4) the impact of the principle which states most productive crops (per acre) be located on more productive land; and (5) impacts of the characteristics of the population on patterning of production. (Quoted in Arunachalam, 1965:12)

In response to a question about the extent to which actual land use patterns adjust to physical factors, Phipps and Dumanski (1983) acknowledge the lack of agreement among writers on the weight that should be given in the U.S. to physical factors. They end up disagreing with many writers about the value of these environmental factors on the locating of wheat production and instead emphasize the impacts of economic and social factors, elements of the farmer's life history, as well as his technical skills as important determiners of the locating of crops (p. 78). Heady and Dillon (1961) also recognize the significance of economics in the locating of crops around the market. The OECD (1979) sees two major forces working in the peri-urban areas--high land prices and increased uncertainty. Whereas the first might discourage wheat production in the interest of perishable crops, the other encourages production of short-term, low-investment wheat production. Hull (1969), on the other hand, sees technological achievement as greatly mobilizing production and reorganizing it so that some crops in effect move from one hemisphere to another. As for the U.S., Hull sees these technological achievements as improving productivity per unit area and inflicting locational changes on agricultural production.

Slater and Levin (1981) see technology as being capable of providing components essential to plant life, therefore making it possible to ignore limits drawn by the environmental limitations when locating crops. This last is a point which the second hypothesis in the present research might have stretched. But it runs contrary to the first hypothesis, which deals with the locating of perishables production, and which posits that 
these locations depend on characteristics of the crops themselves and/or those of the urban market more significantly than on anything else. As such, the low level of association between the environmental variables and perishables production is supportive of the hypothesis. This support is enhanced by the strong associations regarding the urbanization and intensity variables.

\section{PART TWO: CONCLUSIONS}

The conclusions that follow pertain to the specifics of the present research. They are presented as responses to five questions, which represent the five major areas of this study. Answering these questions will define the parameters of the present research, including discussions of both hypotheses and the prominent findings.

(1) To what extent does the design and execution of the present research vary from previous research efforts in this field, and in what ways are these differences significant?

(2) To what extent do the findings agree or challenge theoretical assertions advanced by Von Thunen (1826) and others, particularly Von Thunen's opponent, Sinclair (1967), and to what extent do the present findings initiate a new view on the patterning of peri-urban agriculture?

(3) What is the impact of modern transportation on the patterning of peri-urban agriculture in developed economies, and which crops are most responsive to the distanceto-market factor?

(4) What is the impact of other technological and environmental factors on the spatial distribution of agriculture in the peri-urban zone, and which of these (or what others) most strongly influence the locations of crops in these areas? Of particular impartance here would be the difficult issue of urbanization's role on the patterning of periurban agriculture.

(5) What impact do land use regulations exert on the spatial distribution of peri- 
urban agriculture, and how (if at all) does this impact vary between specific crops?

It is the purpose of this conclusion to address these issues and to provide answers to the questions as fully as the research findings permit.

\section{Characteristics Unique to the Present Research}

There is much in the design and execution of the present research to distinguish it from previous research and study methods in this field. These differences include both an attempt to distinguish between both different classes of crops and within the same class. This runs contrary to the more common practice of handling crops in a generalized fashion. This failure has inclined past research to test little more than the measuring device itself, and not the intended hypothesis.

Instead of testing data for net returns, as in previous research, the present study uses data on total acreage cultivated with the crop in question. This was done because it is thought that figures for acreage more closely reflect the true determinants of production and choice of crops. The exception to this is in the case of greenhouse culture, for which the variable acreage was replaced with the variable number of farms with greenhouses (in the county of analysis). (Unfortunately, most of the data on greenhouse culture are withheld by the Census Bureau in order to maintain the anonymity of the farmers.) It was thought that this adjustment of acreage to numbers is valid in the interest of accuracy and sensitivity of the measuring device, ensuring that the test measures precisely what it is designed to measure. In the present case, this goal was, as stated earlier, to measure correlations with urbanization and related urbanization factors. Notable about this adjustment for greenhouse culture is that it provides a homogeneous measure, without regard to the size of the farm. It was not thought that this homogeneity would create any difficulties in the analysis, since most farms that employ greenhouse techniques are relatively small. At the same time, the kinds of perishable crops that are cultivated in greenhouses are not widely disparate. 
The variabies chosen to measure the correlations of land uses in peri-urban zones were closely interrelated with the items they were to measure. For example, the accessibility variable uses quality and intensity of transport available in a county as a measuring device rather than the traditional variable of linear distance to the urban market, which rarely shows much correlation (Kellerman, 1977). Further, the fertilizer and irrigation variables were both meant to measure the intensity of production and to function as proxies for urbanization and higher land values in the near vicinity of the urban market.

The variable INTERACT proved effective; as stated earlier, it was meant to measure impacts of urbanization on land uses in the peri-urban zones and outer nonmetropolitan urban centers expressed as correlation with the acreage (or number of farms in the case of greenhouse culture) cultivated with the measured crop. On the other hand, the variable URBAN was designed to measure the sum impact of the urban population of the SMSA proper on agricultural land uses in the peri-urban zone. Both variables showed statistical significance and large coefficients. However, each variable proved effective with crops that the other did not--that is, they tended to act alternately.

\section{Comtemporary Determinants of Peri-Urhan Cropping Patterns}

A brief summary of some aspects of previous research in the field will show how the present research differs from previous efforts. Although prior literature on peri-urban agriculture nearly unanimously accepts the cultivation of perishable crops in the peri-urban zone, most writers tie its cultivation to suitability of environmental conditions and/or to shorter distance to the urban market. Where these two conditions are not favorable, researchers in the field are pessimistic about the future of perishable crops in peri-urban areas, even those on the fringe of urban centers with metropolitan status. Grotewald (1959) presents an extreme example of this.

For the production of grain crops, there was again virtual unanimity supporting the 
view that grain production exists nearly exclusively at sites farther out from the market center. Sinclair (1967) may be the sole exception to this opinion; however, Sinclair's reasons for finding exception to the rule do not totally agree with those offered in the present research.

The present research differs from prior efforts in several respects, both for the production of perishables and of grains. For example, one of the hypotheses offered here posits that perishables production is taking place in peri-urban areas because it is attracted by urbanization on the one hand, and because these perishable crops do, in many ways, fit quite nicely the urban environment. Regarding the second hypothesis, which pertains to grain production, the present research differs with most research in the field as it describes and explains the spatial distribution of grain production around the market center. Prior research suggest that grains are extensively grown, that they sell inexpensively per unit product, and that they should therefore be limited to outlying locations. The present research challenges these claims on the grounds that environmental conditions, recently improved market prices for grain crops (the result largely of newly developed international markets for grain), and higher productivity per unit land (the result largely of developments in agricultural technology) permit grain crops much greater access to the near vicinity of the market center, that is, the peri-urban zone.

Another important difference stems from the fact that a great deal of previous research on peri-urban agriculture has attempted to follow too closely the footsteps of Von Thunen (1826). Consequently, it has tended to analyze agricultural production as a lump sum, collectively failing, then, to recognize the significant factors that differentiate within individual crops, including growth requirements, consumption practices, marketing requirements, and many other factors, all of which require that criteria for assessing the probable locating of crops would vary as much as the crops themselves. Recognizing this variability is essential if one expects that the testing instrument will measure precisely what 
it is intended to measure.

Another feature distinguishing the present from past research efforts involves distinctive political and environmental features of commercial agriculture in the United States which enable producers here to exploit specific natural environmental advantages without then being disadvantaged by political boundaries when marketing their produce. These disadvantages, which come in the form of duties or tariffs, may add significantly to production costs, and therefore create a considerable impact on the location of agricultural production. This factor is important insofar as it limits the applicability of the present findings, which are based on agriculture in the U.S., to areas (for example, Europe) where political considerations play a role.

\section{A Sector Theory of Peri-Urban Cropping Patterns}

Application of the present study's data and methods resulted in a sectoral cropping pattern, and not the concentric pattem that is more commonly found in models emerging from a great deal of prior research (see Figures 5 and 6). These sectoral cropping patterns are roughly wedge-shaped. Furthermore, the spatial layout of the cropping patterns varies from one crop to another in a given geographical area. For example, in the case of wheat, the pointed end of the wedge may face toward the market center. In the case of the perishable crops, however, the narrow end of the wedge may well not be pointed, but instead be rather narrow, since the production here is meant almost exclusively for the adjacent market, which in Figure 5 is an SMSA of metropolitan status.

Smaller, nonmetropolitan markets (Figure 6) have sectoral pattems that are also wedge-shaped, in this case allowing the crops to share the necessarily smaller peri-urban areas around the market's fringe. Still, though, the shape representing spatial layout is seen to differ from crop to crop. For example, in the case of vegetables, the narrow end of the wedge points outward, but is narrower in these smaller urban centers; its extension will 
be shorter because more of the produce is meant for local consumption. At the same time they can survive mildly higher land rent in the peri-urban fringes of the smaller urban centers. However, the wedge representing grain (in this case wheat) would point toward the market, in the direction opposite that for vegetables, which reflects the effects of gradually increasing land rents as one approaches the market center. It should be noted, too, that grain produced here is not limited to the local area, but is in fact very widely marketed. Other crops located in the area and serving the local market will be represented by other wedges in accordance with prevailing environmental conditions. Further, islands of both grain and perishables production will occupy some outlying locations, these pockets of production reflecting specific concerns with convenient environmental conditions, access to transportation, and outlying markets (see Figure 6).

\section{Transportation Technology and Peri-Urban Agriculture}

The influence of transportation in the locating of perishable crops was revealed in the coefficient for the variable ACCESS. This variable was large and positive for vegetables production and greenhouse/nursery production. For both, the BETA values for the variable increased in the direction of the market. The variable ACCESS did not show such high or positive correlations with wheat production.

Such disparity between the two crops and their manner of correlating with the variable ACCESS reveals several important things:

(1) The production locations for perishable crops is highly sensitive to access to varied means of transport, so much so that areas with easy access to effective lines of transportation are greatly induced to utilize their peri-urban zones for this kind of agricultural land use. This trend is even more pronounced at locations closer to the market center, since the proximate market center is that area's primary marketing outlet.

(2) Production in peri-urban zones that do not have direct access to varied means of 
transport may opt instead for producing grains, which are indirectly consumed, even when these sites are located only a short distance from the proximate market. It should be recalled that these products are normally marketed nationally and internationally.

(3) Access to national and international markets for grains involves transport over long distances. Accordingly, producers of these crops are concemed less with varied means of transport, and instead require "quality" transport--that is, means of transport that offer low costs for the transport of bulky produce over great distances.

(4) Given the above, correlations between ACCESS and each of these two categories of crops should reflect the sectoral nature of peri-urban agriculture. In fact, the empirical evidence clearly and strongly supports this finding. Production zones are clearly sectoral rather than concentric, contrary to assertions in a great deal of the prior literature in this field. The concentric model, in fact, relied on the assumption that all land in the periurban zone had direct and easy acces to varied means of transport, and ignored the fact that intensity of production could be substituted for other factors of production and/or distribution. Again, the sectoral pattern of production is a direct outcome of access to new technology (including transport technology), and the feasibility of substituting factors of production for factors of distribution.

It must be noted that "agribusiness" in the United States is dominated by a relatively small number of very large companies. These companies maintain extended vertical links and spatial arrangements that enable them to manipulate production and distribution with the involvement of few intermediaries. Arrangements of this kind are thought to offer the most efficient use of resources, including application of the most advanced technologies and the fullest possible optimization of substitution in the production and distribution. This results in lower production and marketing costs and potential increases in retums per unit area for production investments in dollars. 
Technology Variability and Crop Location

Despite the fact that crops may differ from one another in many ways, a number of those tested here showed that such differences should not prevent them from competing effectively against one another for access to the same production locations, and in some cases sharing the same location. This is so largely because each crop exploits characteristics unique to it and/or to characteristics of its surroundings. An important variant which can overtum the traditional expectations in this regard is the matter of access to advanced tecinnology. This access generally works in a package, that is, as a combination of factors such as higher intensity production, bulk-rate transportation, relatively higher market prices per unit product, and easily accessed international markets. For example, substitution of factors makes it possible for low-priced wheat to compete successfully with vegetable and greenhouse production, as well as with other urban-related land uses, for peri-urban sites with high rents per unit area.

Perhaps the most significant of these technological influences are those which contribute to higher intensity production. In fact, these provide by far the most plausible explanation for the expansion of wheat cultivation in the near vicinity of the urban marketplace, where land rents are disproportionately high for a relatively low-priced and extensively cultivated crop such as wheat. Such production intensity is the result primarily of generous application of fertilizer. In fact the variable FERTILIZ carried a coefficient larger than all others in the analysis of wheat production at all locations under study.

Following is how Bond and Umberger (1979) explain the effects of the intensive use of nitrogen fertilizers on wheat production:

Nitrogen fertilizer, the major nutrient applied on wheat, has been known for decades to increase wheat yields in nitrogen-deficient soils. Nevertheless, the widespread use of nitrogen fertilizer on wheat required a reduction in cost .... As a result of the more efficient fertilizer production technology developed in the 1930 s ... prices increased much less than the price of many other inputs. [This] encouraged commercial fertilizer sales with nitrogen ingredients alone... from one million tons in 1950 to over nine million tons in 1970. (p. 79) 
High intensity application of fertilizers for wheat production does not appear to vary considerably with distance from the proximate market. This might indicate the overriding impacts of the national and international markets over the local differences in land rent.

The present discussion is limited to developed countries, particularly the United States, where access to advanced technology is available to the mass of producers. This includes access to inputs, foreign markets, low-cost transportation, as well as other features of technologically advanced agriculture. The discussion also presumes that locational variations are not inhibited by political factors such as tariffs and duties, and that each crop exploits to the fullest the natural environmental advantages of each site.

This higher intensity production made feasible by access to a higher level of technology is reflected in the strong and positive correlation between irrigation and wheat cultivation. Considering that other urbanization-related issues bore negative correlations with this variable, one must conclude that if it was not for the higher intensity production per unit area and the relatively higher market price per unit product--both of which are made possible by means of new technology--it would be unlikely that the commercial cultivation of wheat would have expanded to sites this close to the market center. This assertion is reinforced by the fact that even a slight improvement in the market price of wheat is capable of generating large sums of revenue for the producer. Such price increases, again, are the product of access to the international market, which has been made possible by access to modern transportation technology (see Chapters III and IV and Table I). The impact of technological advancements appears to have created enough changes in the circumstances of wheat production and marketing for the wheat crop to move into locations near the market center and compete successfully against other land uses in the peri-urban zone.

How, then, does this same technology influence the spatial patteming of perishables production, namely fresh vegetables, greenhouse culture, and nursery production? The two variables seen to best represent the impacts of technology on the 
spatial distribution of perishables production are FERTILIZ and IRRIGATE. However, both are missing from the data collected for greenhouse culture and nursery production. However, IRRIGATE is present in the analysis of vegetable cultivation. As seen in Tables $X I$ and XII, this variable has by far the highest $R^{2}$ change among all variables in the analysis in all tiers of counties. Furthermore, this predictor possesses the largest coefficient. Moreover, these values rise much higher as one moves in the direction of the market center. Further, in addition to being an indication of technology, IRRIGATE is also, as has been stated many times earlier, a strong indication of higher intensity production and all that this implies about the ability to compete with land uses in higher-rent areas. In short, perishable crops are distinctly more inclined toward intensified production than are a great many other agricultural products.

It should be noted here that irrigation water is not always used strictly for irrigation. Under certain circumstances it is used to keep temperatures above freezing. In essence, this is an environmental function, in that it is intended to assist with the production of perishables in locations where climatic extremes threaten the viability of the crop's production. Such cultivation becomes even more viable when there is a nearby market for that production. Of course, this is precisely the case in peri-urban areas, particularly those with metropolitan status. Needless to say, though, technology is needed to transfer water, to distribute and apply it, and, more important, for doing all of this at a bearable cost.

The present research has amply demonstrated that new technologies are capable of significantly ameliorating differences that usually exist between various crop categories with regard to their locations relative to the market center. As such, better and more technology might mean more crops competing for the same land sites. That would be more the case for locations in the immediate vicinity of the market. The reasons for this go directly to the matter of the exhaustive extent to which agricultural production exploits characteristics of each crop individually, which is made possible by the availability of more 
technology. It is aiso the resuit of increased riexibility exhibited by many crops regarding their location of production because of their access to more markets. As a result of this, there should be no contradiction between the mixing of production in the immediate vicinity of the market and a more regional approach to production at outer locations. The spatial outcome of such arrangements should fit nicely with the sectoral pattem of peri-urban agriculture advanced in the present research.

In accordance with the above, the fact remains that access to new technologies means higher intensity production at lower cost. This means a competitive edge for some

crops, which tends to draw them closer to the market center. Such forward motions would be costly without the advanced technology. When combined, these findings make it appear as if new technologies are distributing added values to some crops at the expense of others. In fact, this might not be far from the truth, particularly with respect to the commercially produced crops.

\section{The Intensity of Crop Production}

A significant by-product of the sectoral patterning of peri-urban agriculture pertains to the intensity of production. Findings presented in the present research dispell a great deal of uncertainty that has surrounded this issue for a long time. One such uncertainty relates to the question of whether or not production intensity should be considered a theory that is separate and distinct from a theory of cropping patterns. As discussed in Chapter II, this issue is a prominent one in the field. The findings of the present study, however, strongly indicate the following.

Intensity does not constitute an independent theory, as has been asserted for some time by other researchers--those who both agree with and disagree with Von Thunen's view of agricultural cropping patterns. The present outright rebuttal of the initiative toward a theory of intensity rests on this study's new sectoral patterning of peri-urban agriculture. 
This is so largely because eariier claims that intensity is in fact an independent theory are almost exclusively tied to theory espousing a concentric pattern of production. And this, in turn, rests on the assumption that each and every production zone should have its own specific and unified level of intensity that fully encircles the market at the center of the concentric pattern. This view is a result of the prior assumption that noncompatible crops (i.e., crops conventionally assigned to different production zones) could not be mixed in any single production zone.

The above assumptions have been undermined by the present study and its sectoral pattern of production. The justification for this revision rests on the finding that peri-urban sites are availing themselves more and more readily to a package of factors for cultivating and distributing crops that rely to a growing extent on new technology. This creates a rapidly changing formula for cost-effective agricultural production in the peri-urban zone. This new formula is distinguished by a heavier reliance on substituting factors both of production and distribution. The ultimate aim is more competitive output--lower cost per unit product.

Access to new technology, then, makes it possible for entrepreneurs to manipulate production and distribution factors to their best advantage. This may take the form of increasing yields, lowering costs, or even locating crops in unconventional sites. The result of this is mixed production and new patterns of distribution, where a crop could feasibly occupy sites at two different locations in different production zones. This would require different levels of production intensity, of course, that would be achieved by trading-off other factors of production and/or distribution.

There is strong disagreement among supporters of an "intensity theory" on more than one issue. One of these is whether the intensity gradient rises smoothly in the direction of the central market, or whether it is staggered, varying with the crop under cultivation. In fact, these groups even disagree on whether this intensity gradient rises in 
the direction of the market center, or instead in the opposite direction.

The present assertion that intensity is not an independent theory is supported in the present research in yet another way, which is grounded on the present findings showing a rather diffused intensity pattern that is affected largely by the kind of crops cultivated and their location relative to the market center. Still, the findings show that perishables production has an intensity gradient that rises continuously in the direction of the market. This is true both for vegetable crops and greenhouse culture. However, wheat production has a diffused production intensity pattern that does not appear to relate to the crop's location relative to the market center. This diffused intensity pattern supports Von Thunen's view, since he recognized varied levels of production intensity for different crops. In fact, Von Thunen assigned more than one intensity level for the same crop if and when that crop was cultivated at different distances from the market center (see for example grain production in tiers 3,4, and 5 of the Isolated State).

The intensity gradient for perishables picdiction, which rises in the direction of the market, supports the first hypothesis of the present study. This is so in that it reflects the inclination of perishables to compete for sites in the immediate vicinity of the market when sufficient intensity of production is possible. This intensity criterion also holds for grain producers in the near vicinity of the market. However, for grain producers the distinction between outer and inner sites in not that clear, since package technology makes it possible for them to substitute other factors of production and/or distribution for intensity. Contrasting the strong correlation between the variable ACCESS and perishables production with the same variable's correlation with wheat production indicates that in no way does one level of intensity systematically encircle the market (concentrically) simply because no single crop could alone claim that production zone for itself.

These findings strengthen the argument for considering intensity as a factor of production which is ancillary to the characteristics of the crop itself. Still, it is a factor that 
is flexible enough to allow producers to manipulate it to infiuence production locations, even though it must be accepted as an intregal part of a larger package of factors. Consequently, the impact of the variable intensity on the spatial distribution of agriculturai production must be analyzed in the context of the entire package of factors, and not as a single-variable response to added-inputs.

\section{The Role of the Environment in the Location of Peri-Urban Agriculure}

The environmental variables show a low correlation with both crops, except for the variable GROWTH, which is positively associated with greenhouse products. The significance of this variable was confirmed, however, when the wheat region was divided into homogeneous subregions. This was supported by findings for the variable PRECIPIT, which also resulted from conducting the analysis on the basis of subregions. The new findings showed both variables to be statistically significant in only two subregions (see Tables VII and XIII). The conclusion here is that the role of the natural environment in the locating of crops in the peri-urban zone, although continuing to make differences in yields and production costs, will be strongly overshadowed by the willingness of commercial agriculture to take risks of crop failure and/or bear higher production costs per unit product in order to enjoy the locational advantages provided by proximity to the market center and/or gains for a more marketable product.

\section{The Role of Urbanization on the Lecation of Peri-Urban Agriculture}

For all crops the impacts of urbanization expressed through the predictors URBAN and INTERACT were independent of the impacts brought about by technology. For example, although new technologies did enable wheat production to occupy locations in the market vicinity, the negative sign associated with the predictor URBAN, with its high coefficient, continued to show the incompatibility of this land use with urbanization. This was reversed for vegetable crops and greenhouse culture, with the signs for the variables 
INTERACT and URBAN turning positive with large coefficients. However, INTERACT showed statistical significance only with vegetable cultivation, whereas URBAN showed statistical significance with greenhouse production. Among other things, this indicates the complex nature of urbanization and suggests that these compound impacts are affected by an array of variables. A multi-variable approach miust be used even when investigating the spatial distribution of just one crop category, since changing production locations rearranges the priorities of analysis.

\section{Land Use Regulations and the Location of Peri-Urban Agriculture}

The variable ZONING represents mandatory land use regulations enacted for the preservation of agricultural land against urban encroachment. This study's findings indicate the distinctly urban nature of this variable. This is clear in the high BETA values for the variable's association with greenhouse culture and nursery production. This trend is most pronounced in county tiers having metropolitan status. This variable's correlation with vegetable production is similar at the SMSA level. Interestingly, no such levels of statistical significance emerged for this variable for areas cultivated with wheat, either at the SMSA or metropolitan levels of counties.

These findings reflect the true urban nature of ZONING and, consequently, the close association between perishables production and land use regulations. Still, it is quite difficult to determine if there is a cause-effect relationship between the two. Moreover, if in fact a causal relationship does exist, it is equally impossible to determine which is the cause and which the effect. In other words, although it is possible that land use regulations come after the fact, that is, to protect existing perishables production in the peri-urban zone, it is equally possible that perishables production is drawn to these areas as a consequence of the passing of adventageous land use regulations. Regardless of these uncertainties, however, it is clear that a strong association exists. 


\section{The Research Findings and Present Theory}

It would be a gross oversimplification to say simply that the research findings either agree or disagree with present theory. However, some viable observations on this issue may be reasonably made.

The present research has confirmed that vegetable production is suited to peri-urban areas, although for reasons that go far beyond the distance-to-market factors proposed by Von Thunen. Instead, the present research found that it was higher intensity production and its positive association with urbanization that distinguished perishables production in the high-rent areas near the market center. However, the findings also showed the feasibility of perishables production at more distant locations while at the same time noting the positive impact of the distance-to-market factor on perishables production in the periurban zone.

The present research confirms that higher intensity production made possible by new technologies makes feasible the cultivation of wheat in the peri-urban zone. This finding disagrees with Von Thunen's, of course. This finding also disagrees with Sinclair (1967), who ascribes such forward moyement of grains to land speculation and the transitional nature of agricultural production at the advanced sites. The present study, however, asserts that grain production in the peri-urban zone is not transitional, but is there to stay. This is due primarily to higher intensity production per unit area which added inputs makes possible, and to low transportation costs and improved market price.

\section{Technology and the Location of Peri-Urban Agriculture}

New technologies have had a significant influence on the pattering of crops in the peri-urban zone, largely as a result of substitution. These impacts are expressed in the findings in more than one way. The findings make clear, for example, that high intensity production in the peri-urban zone is not limited to just perishables, as current theory often 
claims. Wheat, with the assistance of added inputs like fertilizer and irrigation, may be cultivated every bit as intensively as perishables, and may therefore compete for the same land sites as are commonly occupied by vegetable farms, even those in the immediate vicinity of the urban market. On the basis of this finding, the present study advanced a sectoral pattem of distribution for peri-urban agriculture instead of the more common zonal pattern upheld by theorists over the years. Sectoral patterning of this kind makes possible the mixing of more than one crop in a given agricultural zone--including the zone in the area most immediately surrounding the urban market and which theorists commonly reserved exclusively for perishables production.

Grain production at these locations may reasonably result from calculated business decisions that are based on the assumption that such farming is economically feasible. However, such feasibility requires access to "convenient" transportation. The findings show that access to merely "varied" means of transportation was not particularly significant to siting decisions. Instead, as the literature indicates, focal is access to "specific" transport, namely, low-cost bulk carriers. Of course, transportation is not the single factor encouraging grain production in the peri-urban zone. Also important are new technologies.

The findings show that access to "varied" means of transport is also statistically significant in the locating of perishable production, particularly for perishables from greenhouse cultivation. Moreover, this significance becomes more pronounced the nearer one approaches the market center. This last finding confirms the significance of the distance-to-market factor, which was hypothesized to encourage perishables production in the near vicinity of the urban market, despite advances in transportation and food preservation technology which have nade distant agricultural sites feasible. This significance confirms the sectoral nature of crop production patterns in the peri-urban zone.

The intensity of production is as important as crop category in determining crop location around the urban market, since it directly affects yields and revenues from 
agricuiture. The results show that intensity of production is pitted against environmental suitability in influencing crop siting decisions, since it appears that some producers are willing to overlook the threat of some environmental inconveniences in order to seek higher productivity through added inputs. This trend is more pronounced in perishables production than with grain production. For vegetable production, for example, the BETA values associated with environmental variables are statistically insignificant and/or show a minus sign, which indicates weak and inverse associations with vegetable production. Regardless, these locations witnessed the cultivation of large tracts of vegetable crops. This may be explained by the BETA with a positive sign for the variable IRRIGATE, which indicates the extent to which added inputs substitute for environmental suitability. Similarly, the variable INTERACT, which is a proxy for urbanization, also showed high BETA values with positive signs, an indication of the strong association between these kinds of crops and the nearby urban population. These findings also indicate the willingness of producers to accept some risks of crop failure arising from inconvenient environmental condition in order to locate close to urban markets. The same conclusions could be drawn for greenhouse culture.

In the case of grain production, though, environmental conditions proved to be more significant, although not so strong that producers were not willing to employ added inputs and accept higher production costs.

\section{PART THREE: IMPLICATIONS OF THE PRESENT RESEARCH}

\section{The Findings}

Following is a brief synopsis of the primary findings of the present research. This study advances a sectoral pattern for commercial agriculture in the peri-urban zones in the United States, where access to varied markets and natural environmental conditions in not hampered by the effects of political boundaries. This new sectoral pattem 
of production is meant to replace the concentric/zonal patiern first advanced by Von Thunen in the Isolated State and since upheld by most researchers in the field. Moreover, the present research convincingly argues against an intensity theory of production, since the basis of this "theory" depends upon the concentric model of production patterns, which this study disputes. Instead, intensity is viewed here as one element of a "package" of production factors that new technology makes available to producers. This production package allows the producer to manipulate production intensity factors by means of substitution.

The present research argues for a diffused intensity gradient that would vary in its direction according to the crop under cultivation and the location of production. This finding is similar to Von Thunen's, who found perishables production's intensity gradient to rise in the direction of the market, while grain production's gradient was found to be more diffuse. These findings contradict those of other researchers, particularly Sinclair (1967), who argues that the production intensity gradient invariably drops in the direction of the market. The author's findings are reflected in the attraction exerted by urban markets on perishables production.

This study also established convincingly the importance of the value of land per acre as a determinant of the patterning of peri-urban agriculture. As far as this author is aware, the present study is the first to introduce this variable into a study of this kind and obtained meaningful results. Similarly, this study was the first to advance the notion that transportation (viz á viz accessibility) was a variable affecting agricultural siting in periurban zones. Prior research invariably approached this variable as either a linear-distanceto-market variable or as a cost/distance one. Instead, the present study measured the county's accessibility to varied means of transportation with interstate capabilities. This measurement magnified the individual differences between the crops under study as well as identifying the market destination of the crop. 
This study also recognized the complex natures of urbanization and the natural environment as variables, and to account for the complexity assigned two variables for each set of factors. The outcome justified the choice. It was noted, for example, that the variable INTERACT, which was to represent suburban populations of SMSAs or of county seats showed impacts that varied significantly from those of strictly urban populations of the SMSAs proper. To the best of this author's knowledge, this is the first time differentiations of this kind have been made.

It has been generally taken for granted that grain is cultivated extensively. Even those who lately recognized that it was in some cases cultivated intensively stopped short of advancing the notion that it might be produced at sites near the market center with high land rents, except as an expedient in cases of real estate speculation where the land was awaiting urbanization. This research found quite differently, and this finding should open new doors to research on this issue.

\section{Discussion of the Study Design and Recommendations for Future Research}

In this author's view, the present findings might be most accurately described as having added new issues to the list of factors that must be considered to be determinants of the spatial distribution of peri-urban agriculture. At the same time, they raise doubts about issues that have been taken for granted for quite some time.

Important here is the matter of the testing region. In the present case the research region was perfectly suited to the hypotheses in many respects. The study's advancement of the new sectoral pattem of production is a case in point. The study area allowed a clear determination of this new pattem regardless of the scale or region of application so long as the analysis is conducted in a developed economy with access to reasonably advanced technology. Secondly, this study raised suspicions about the way some variables have been used in the past and proposed new ways of introducting them into an analysis which 
may prove useful to future researchers. Third is the matter of scaie. Unilike a good deal of research on this subject, which sees no substantive differences in outcome regardless of the scale of the analysis, this author is convinced otherwise. Any set of variables introduced into an analysis must have as its most important criterion of selection the scale of its application.

Accordingly, this author recommends that researchers employ the micro scale when dealing with the market-size analysis. It would not surprise this author to find that the outcome of such research disagreed with the present findings. It might even prove to be the case that a micro-scale analysis might salvage the Isolated State in more than one way.

\section{The Isolated State in a Sectoral View of Agricultural Production}

As a theory of crop production in the peri-urban zone, the Isolated State is in many ways viable, having successfully weathered the impact of the lapse of time and the development of new technology. Following is a synopsis of some of the ways in which this is true.

(1) This study shows clearly the advantages of cultivating perishable crops in the immediate vicinity of the urban market. This is seen to be the case even in technologically advanced countries with simple and inexpensive access to a wide range of markets via sophisticated transport infrastructures.

(2) The central theme of the Isolated State which pertains to the impact of shorter distances to the market on the location of perishables production is generally supported in this study. At the same time, though, it is seen that the impacts of shorter distances are not necessarily binding, largely because of the extreme flexibility permitted producers by transportation, agricultural (i.e., added inputs), and food-preservation technologies.

(3) Von Thunen recognized the importance of considering the size of the urban center in any analysis of this kind, as well as considering various characteristics of the 
consuming public. All of these factors had an impact on crop distribution in the immediate vicinity of the market in Von Thunen's time, and continue to do so today.

(4) Despite the new sectoral pattern of crop production advanced here, the importance of Von Thunen's concentric pattern cannot be dismissed lightly. Its clarity was exemplary for the ease and spontanaity with which it conveys some basic information about the nature of agricultural production, the urban market, and competition for land in the periurban zone. The new sectoral pattem cannot make such claims, largely due to the tradeoffs, substitutions, and other technology-induced influences, discussed above, which resulted in the sectoral model in the first place. It requires more than a simple model to explain the economic viability of cultivating a crop in a high rent area because of proximity to an urban market that does not consume that crop. 


\section{BIBLIOGRAPHY}

Alcaly, Roger E. 1976. "Transportation and Urban Land Values: A Review of the Theoretical Literature." Land Economics, 52(1): 42-53.

Alcock, M. B., et al. 1974. "Measurement, Evaluation and Management of Climatic Resources for Grassland Production in Hill and Land Areas. In James A. Taylor (Ed.), Climatic Resources and Economic Activity. New York: John Wiley \& Sons.

Alexander, J. W., 1963. Economic Geggraphy. Englewood Cliff, NJ: Prentice-Hall.

Alexander, J. W., et al. 1967. "Freight Rates: Selected Aspects of Uniform and Nodal Regions." In Readings in Economic Geography, Howard G. Roepke (Ed.), New York: John Wiley and Sons, pp. 552-569.

Allan, W. 1965. The African Husbandsman. Edinburgh: Oliver \& Boyd.

Alonso, W. 1960. "A Theory of the Urban Land Market." Papers and proceedings of the Regional Science Association, 6:149-157.

Alonso, W. 1964. Location and Land Use. Cambridge: Harvard U. Press.

Amiran, D. H. K. 1965. "Arid Zone Development: A Reappraisal Under Modern Technological Conditions." Economic Geography, 41(3):189-210.

Applebaum, Richard P. 1978. Size, Growth, and U.S. Cities. New York: Praeger.

Arunachalam, B. 1965. Economic and Commercial Geography. Bombay: A. R. Sheth.

Baker, O. E. 1921. "The Increasing Importance of the Physical Conditions in Determining the Utilization of Land for Agriculture and Forest Production." Annals of the Association of American Geographers, 11:17-46.

Baldridge, Don and Howard Bowman. 1987. "Spring Wheat Variety Performance." Montana Farmer Stockman, 74(9):8.

Balint, A. (Ed.). 1970. Protein Growth by Plant Breeding. Budapest: Akademiai Kiado/Univ. of Agricultural Sciences.

Ball, S. 1975. "The Rural Urban Fringe: A Case Study of Dighori." The Deccan Geographer, 13:217-225.

Batie, Sandra S. and Robert G. Healy (Eds.). 1980. The Future of American Agriculture as a Strategic Resource. Washington DC: The Conservation Foundation.

Beek, K. J. 1978. Land Evaluation for Agricultural Development. Wageningen, Netherlands: International Institute for Land Reclamation and Improvement. 
Belkin, Lisa. 1985. "Choices: Do American Consumers Have Too Much of a Good Thing." The Oregonian. 135 (Sept. 21):D1.

Bennett, H. H. 1963. "Adjustment of Agriculture to Its Environment." Annals of the Association of American Geographers, 33:163-198.

Bemard, Harold W., Jr. 1980. The Greenhouse Effect, Cambridge, MA: Ballinger.

Berry, B. J. L. 1968. Theories of Urban Location. Association of American Geographers, Resource Paper No. 1. Washington DC: Commission on College Geography.

Berry, B. J. L. 1972. "Hierarchical Diffusion: The Basis of Developmental Filtering and Spread in a System of Growth Centers." In Niles M. Hansen (Ed.), Growth Centers in Regional Economic Development. New York: Free Press.

Besant, L. and M. T. Katherine. 1973. Grain: Production, Processing Trading. Chicago: City of Chicago, Board of Trade.

"Best Bets in '86: Raise Com for \$1.80." Farm Joumal 110(1):13-15.

Bills, Nelson R. 1979. "Peri-Urban Agriculture in New York State." In Agriculture in the Planning and Management of Peri-Urban Areas, Vol. II, case studies presented by the Organization for Economic Cooperation and Development. Paris: OECD.

Blair, A. A. 1980. "Urban Influences on Farming in Essex. Geofonum. 11:371-384.

Blakley, Leo L. 1981. "Regional Markets for Agricultural and Food Products: Needed Research. Southern Journal of Agricultural Economics, 13(1):69-81.

Boesch, H. 1964. A Geography of World Economy. New York: Van Nostrand.

Bond, J. J. and D. E. Umberger. 1979. Technical and Economic Causes of Productivity Changes in U.S. Wheat Production. 1949-76. U.S. Dept. of Agriculture Technical Bulletin No. 1598. Washington DC: GPO.

Borchert, John R. 1972. "American Changing Metropolitan Regions. Annals of the Association of American Geographers, 62(2):352-374.

Boserup, E. 1965. The Conditions of Agricultural Growth: The Economics of Agrarian Change Under Population Pressure. Chicago: Aladine.

Bowler, I. R. 1981. "Some Characteristics of an Innovative Form of Agricultural Marketing." Area. 13:307-314.

Boyce, Ronald R. 1980. Geegraphy as Spatial Interaction. Seattle: U. of Washington.

Brookfield, H. C. 1972. "Intensification and Disintensification in Pacific Agriculture: A Theoretical Approach." Pacific Viewpoint, 13:30-48.

Bryant, C.R., L. H. Russwurm, and A. G. McLellan. 1982. The City's Countryside: Land and Its Management in the Rural Urban Fringe. New York: Longman Group. 
Buchanan, R. O. 1959. "Some Reflections on Agricultural Geography." Geography, 44:113.

Candioti, E. 1986. "Argentines Puzzled by U.S. Joining Farm Subsidizers." The Oregonian, 136 (Aug. 14):D13.

Carver, I. H. 1979. "Policies and Measures for Adapting the Agricultural Sector in PeriUrban Areas to its Specific Functions." In Agriculture in the Planning and Management of Peri-Urban Areas, Vol. II. Case studies presented by OECD member countries and reports on selected studies. Paris: OECD.

Chicago Board of Trade. 1973. Commodity Trading Manual.

Chicago Board of Trade. 1981. 1980 Statistical Annual of Cash and Futures Data: Grains. Metals, Forest Products.

Chicago Board of Trade. 1984. 1983 Statistical Annual of Cash and Futures Data: Grains. Metals. Forest Products.

Childe, Gordon. 1950. "The Urban Revolution." Town Planning Review, 21:4-7.

Chisholm, Michael. 1968. Rural Settlement and Land Use. London: Hutchinson U. Library.

Cohen, R. L. 1956. The Economics of Agriculture. London: John Nisbet.

Conkling, Edgar C., and Maurice Yeates. 1976. Man's Economic Environment. New York: McGraw-Hill.

Conzen, Michael P. 1977. "The Maturing Urban System in the United States, 1840-1910." Annals of the Association of American Geographers, 67(1):88.

Coppock, J. T. 1964. "Crops, Livestock, and Enterprise Combinations in England and Wales." Economic Geography 40:65-81.

Coughlin, R. E. et al. 1981. The Protection of Farmland: A Reference Guidebook for States and Local Govemments. A National Agricultural Land Study, Department of Agriculture. Washington DC: GPO.

Cromley, Robert G. 1982. "The Von Thünen Model and Environmental Uncertainty." Annals of the Association of American Geographers, 72(3):404-410.

Csaki, Norbert. 1974. "Land Supply and International Specialization in Agriculture." In Geography of World Agriculture (3). Budapest: Akademiai Kiado.

Cumberland, K. B. 1964. "Land Problems in New Zealand: A Review." The Geographical Review, 36(1):137-142.

Datoo, B. A. 1978. "Toward a Reformulation of Boserup's Theory of Agricultural Change." Economic Geography, 57:135-145. 
De Lisle, David De Garvis. 1982. "Effects of Distance on Cropping Patterns Internal to the Faim." Annals of the Association of American Geographers, 72:88-98.

Dixit, V. G. 1975. "Land Use in Kolar Doab." The Deccan Geographer, 12:204-208.

Dudal, R. 1982. "Land Degradation in a World Perspective." Ioumal of Soil and Water Conservation, 37:245-249.

Dunn, Jr. Edgar S. 19j4. The Location of Agricultural Production. Gainsville: U. of Florida Press.

Dunn, Jr. Edgar S. 1983. The Development of the U.S. Uroan System. Volume 2; Industrial Shifts and Implications. Washington DC: Resource for the Future (Distributed by Johns Hopkins U. Press).

Eaton, J. A. 1973. Gardening Under Glass. New York: Macmillan.

Evans, L. T. 1983. "Raising the Yield Potential in Genetic Engineering of Plants." In Tsune Kosuge et al., Genetic Engineering of Plants: An Agricultural Perspective. New York: Plenum Press.

Frowine, Steven A. 1986. "Vegetables, Omamentally Speaking." Beds and Borders, 42:28-31.

Furuseth, O. J., and John T. Pierce. 1982. Agricultural Land in an Urban Society. Washington DC: Association of American Geographers.

Gallimore, W. W. 1983. "Transportation Handles the Surge in Production." In Food From Farm to Table: The 1982 Yearbook of Agriculture. Washington DC: U.S. Department of Agriculture, GPO.

Garrett, J.T. and John W. Kelly. 1987. "In the Greenhouse." American Vegetable Grower, Western edition, 35(1):56.

Garrison, William L. and Duane F. Marble. 1957. "The Spatial Structure of Agricultural Activities." Annals of the Association of American Geographers, 47(2):137-145.

Gee, Kerry C., Ron N. Van Arsdall, and A.G. Ronald. 1979. U.S. Federal Beef Production Costs. 1976-77. and Industry Structure. U.S. Dept. of Agr. pub. ERS424. Washington DC: GPO.

Gertler, Len. 1978. Human Settlement Issues, I: Habitat and Man. Vancouver, B.C.: U. of British Columbia Press.

Gibson, Jr., W. L., R. T. Hildreth, and Gene Wunderlich. 1966. Methods for Land Economics Research. Lincoln: U. of Nebraska Press.

Golubev G. and I. Shuytov (Eds.). 1982. Modeling Agricultural-Environmental Processes in Crop Production. Laxenburg, Austria: International Institute for Applied Systems Analysis. 
Gottmann, Jean. 1961. Megalopolis: The Urbanized Northeastern Seaboard of the United States. New York: Twentieth Century Fund, Inc.

Gray, William, H. 1984. Peri-Urban Agriculture in Westem Washington: An Identification of Factors of Potential influence to the Development of Metropolitan Food Policy. Unpublished coctoral dissertation, Portland State University.

Greb, B. W. 1979. "Reducing Draught Effect on Croplands in the West-Central Great Plains." U.S. Dept. of Agr. Information Bulletin 420. Washington DC: GPO.

Gregor, Howard F. 1957. "Urban Pressures on Califomia Land." Land Economics, 33:311-325.

Gregor, Howard F. 1974. "An Agricultural Typology of California." In Geography of World Agriculture (4). Budapest: Akademiai Kiado.

Grennes, T., R. J. Paul, and Marie Thursby. 1978. The Economics of World Grain Trade. New York: Praeger.

Grigg, David. 1969. "The Agricultural Regions of the World." Economic Geography, 45:95-132.

Grotewold, Andreas. 1959. "Von Thünen in Retrospect." Economic Geography, 35:346356.

Hall, Peter G. (Ed.). 1966. The Isolated State, by Johann Heinrich Von Thunen. English edition of Der Isolierte Staat, tr. by Carla Wartenberg. New York: Pergamon Press.

Haninig, Michael D. 1979. "regional Acreage Response by Quarter for Fresh Tomatoes: An Example of the Use of Mixed Estimation." Southern Joumal of Agricultural Economics, 2(2):69-73.

Harlan, J. R. 1980. "Crop Moncculture and the Future of American Agriculture." In The Future of American Agriculture as a Strategic Resource, by S. S. Batie and G. H. Robert. Wahsington DC: The Conservation Foundation.

Harris-Moran Seed Company. 1986. The 1987 Vegetable Growers' Seed Guide. Rochester, NY.

Hart, John F. 1976. "Urban Encroachment on Rural Areas." The Geographical Review, 66:3-17.

Hart, John F. 1977. "The Demise of King Cotton." Annals of the Association of American Geographers, 67:307-322.

Hart, John F. 1980. "Land Use Change in Piedmont County." Annals of the Association of American Geographers, 70:307-322.

Harvey, D. W. 1966. "Theoretical Concepts and the Analysis of Agricultural Land-Use Patterns in Geography." Annals of the Association of American Geographers, 56:361-374. 
Heady, E. O. 1952. Economics of Agricultural Production and Resource Use. Englewood Cliff, NJ: Prentice-Hall.

Heady, E. O. (Ed.). 1971. Economic Models and Quantitative Methods for Decisions and Planning in Agriculture. Proceedings of theEast-West Seminar. Ames, Iowa: Iowa State U. Press.

Heady, E. O. and John L. Dillon. 1961. Agricultural Production Functions. Ames, Iowa: Iowa State U. Press.

Heady, E. O., et al. 1965. Roots of the Farm Problem: Changing Technology, Changing Capital Use, Changing Labor Needs. Añies, Iowa: Iowa State U. Press.

Healy, R. G. and John S. Rosenberg. 1979. Land Use and the States. Baltimore: Johns Hopkins U. Press.

Heid, W. G., Jr. 1979. U.S. Wheat Industry, AER-432. U.S. Dept. of Agr., Economic Statistics Coop Service. Washington DC: GPO.

Heilbrun, James. 1981. Urban Economics and Public Policy. New York: St. Martin's Press.

Hjort, H.W. 1978. "Foreign Demand and Export Potential for U.S. Farm Products." In World Agricultural Trade: The Potential for Growth. Kansas City: Federal Reserve Bank of Kansas City.

Hodder, B. W., and Roger Lee. 1974. Economic Geography. New York: St. Martin's Press.

Hoffman, L. A., P. P. Boles, and T. Q. Hutchinson. 1975. Livestock Trucking Services: Quality, Adequacy and Shipment Patterns. U.S. Dept. of Agr. pub. ERS-312. Washington DC: GPO.

Hoover, Edgar M. 1948. The Location of Economic Activity. New York: McGraw-Hill.

Horvath, Ronald J. 1967. "Von Thünen and Urban Sprawl." Annals of the Association of American Geographers, 57:811-812.

Huff, James O. 1981. "Rich Man, Poor Man in Von Thünen's Isolated State." Economic Geography, 57:127-134.

Hugget, Peter. 1967. "Three Pioneers in Locational Theory." The Geographical Joumal, 133(3):357-359.

Hull, Oswald. 1969. A Geography of Production. London: St. Martin's Press.

Hunt, Thomas F. 1905. The Cereals in America. New York: Orange Judd.

Huntington, C. C., and Fred A. Carlson. 1931. Environmental Basis of Social Geography. New York: Prentice-Hall. 
Hutchinson, Sir Joseph. 1972. Farming and Food Supply: the Interdependence of Countryside and Town. Cambridge: Cambridge U. Press.

Ikerd, J. E. 1985. "The Internationalization of U.S. Agriculture: Policy Implications for the Future." Grain and Feed Journals, 143 (June):14-16.

Isard, Walter. 1956. Location and Space-Economy: A General Theory Relating to Industrial Location, Market Areas, Land Use, Trade, and Urban Structure. Cambridge, MA: MIT Press and John Wiley \& Sons.

Jamison, John A. 1968. Consumer Food Costs: A Comparative Study of Honolulu and San Francisce Bay Area. Stanford, CA: Food Research Institute, Stanford U.

Johnson, A. J. 1970. Organization of Space in Developing Countries. Cambridge, MA: Harvard U. Press.

Johnson, J. F. 1969. "The Influence of Cost Distance Factors on the Overseas Export of Com From the United States." Economic Geography, 45:170-179.

Jones, Alan P, William J. McGuire, and Ann Witte. 1978. "A Re-examination of Some Aspects of Von Thunen's Model of Spatial Location." Joumal of Regional Science, 18:1-15.

Jones, Donald W. 1982. "Location and Land Tenure." Annals of the Association of American Gegraphers, 72:314-331.

Jordan, Terry G. and Lester Rowntree. 1979. The Human Mosaic: A Thematic Introduction to Cultural Geography. New York: Harper \& Row.

Jumper, Sidney R. 1969. "The Fresh Vegetable Industry in the U.S.A.: An Example of Dynamic Interregional Dependency." Tijdschrift Voor. En. Soc. Geegrafie, 60(5):308-318.

"Just What is a Farm, Any'vay?" 1987. Utah Farmer-Stockman, 107(2):9.

Katzman, Martin T. 1974. "The Von Thünen Paradigm: The Industrial-Urban Hypothesis and the Spatial Structure of Agriculture." American Joumal of Agricultural Economics, 56(4):683-696.

Kellerman, Aharon. 1977. "The Pertinence of the Macro-Thünen Analysis." Ecconomic Geography, 53:255-264.

Kerby, Joe Kent. 1970. Essentials of Marketing Management. Cincinnati: Southwestern Publishing Co.

Klages, K. H. W. 1949. Ecological Crop Geography. New York: Macmillan.

Klassen, Perry. 1973. "Regional Compe ative Advantage in the United States." Joumal of Regional Science, 13(1):97-105.

Klassen, Perry, and Tom Arthur. 1987. "Midwest Vegetables--California Style." American Vegetable Grower. Western Edition, 35(1):8-9. 
Kohn, Clyde F. (Ed.). 1961. Urban Responses to Agricultural Change. Iowa City: State U. of lowa.

Kosuge, T., et al. (Eds.). 1983. Genetic Engineering of Plants: An Agricultural Perspective. New York: Plenum Press.

Kresage, David T. and Daniel A. Seiver. 1978. "Problems of Regional Economic Development; Planning for a Resource-Rich Region: The Case of Alaska." American Economic Association 68(2):99-104.

Krueger, R. R. 1959. "The Disappearing Niagara Fruit Belt." Canadian Geographical Joumal, 58:102-114.

Krueger, R. R. 1978. "Urbanization of the Niagra Fruit Belt." Canadian Geographer. 22:179-194.

Krzyminski, James. 1978. Agricultural Transportation: The National Policy Issues. Washington DC: National Planning Association.

Krzymowski, Richard. 1928. "Graphical Presentation of Thünen's Theory of Intensity" (P. G. Minneman, tr.). Journal of Farm Economics, 10(4):461-483.

Kusinski, Witold. "Relations Between Land Use Intensity and the Size of Towns in Poland." Geographica Polonica, 37:121-133.

Laut, Peter. 1968. Agricultural Geography. Vol, 1: Systems. Subsistence, and Plantation Agriculture. Melbourne, Australia: Thomas Nelson.

Laut, Peter. 1968. Agricultural Geography, Vol. 2: Mid-Latitude Commercial Agriculture. Melbourne, Australia: Thomas Nelson.

Leaman, J. Harold, and G. C. Conkling. 1975. "Transport Change and Agricultural Specialization." Annals of the Association of American Geographers, 65(3):425-432.

Leath, M. N., Lynn H. Meyer, and Lowell D. Hill. 1982. U.S. Corn Industry. U.S. Dept. of Agr. publication ERS-479. Washington DC: GPO.

Lessinger, J. 1956. The Determination of Land Use in Rural-Urban Transition Areas:A Case Study in Northem Santa Clara Valley, Califomia. Ph.D. dissertatiion, U. of California, Berkeley.

Lewis-Beck, Michael S. 1980. Applied Regression: An Introduction. Beverly Hills: Sage Publications.

Lloyd, Peter E., and Peter Dicken. 1977. Location in Space. New York: Harper \& Row.

Lu, Yao-Chi, and Leroy Quance. 1979. Agricultural Productivity: Expanding Limits. U.S. Dept. of Agr. Economics, Statistics and Cooperative Service and Agricultural Information Bulletin 431. Washington DC: GPO. 
Malin, James C. 1973. Winter Wheat in the Golden Belt of Kansas. New York: Gctagon Books.

Martin, Michael V. 1987. "Internatinal Trade." Washington Farmer-Stockman, 112(1):5.

Mighell, Ronald L., and John D. Black. 1951. Interregional Competition in Agriculture. Cambridge, MA: Harvard U. Press.

Miles, Guy H. 1974. The Federal Role in Increasing the Productivity of the U.S. Food System. Minneapolis: North Star Research Institute.

Miles, Guy H. 1977. Altemative Food Delivery Systems: An Exploratory Assessment. Baton Rouge, Louisiana: Experimental Resource Group.

Morgan, W. B., and R. C. Munton. 1971. Agricultural Geography. Bungay, Suffolk: Richard Clay/Chaucer Press.

Mosher, Arthur T. 1966. Getting Agriculture Moving: Essentials for Development and Modernization. New York: Frederick A. Praeger.

Muller, Petter O. 1973. "Trend Surfaces of American Agricultural Pattems: A MacroThünen Analysis." Economic Geography, 49:228-240.

Murphey, Rhoads. 1982. The Scope of Geography. New York: Methunen.

Naegely, Stella K. 1987. "Know Your Markets." American Vegetable Grower: Westem Edition. 35(1):10-12.

National Oceanic and Atmospheric Administration (NOAA). 1981. Weather of U.S.Cities. 2 Vols. Detroit: Gale Research Company.

National Oceanic and Atmospheric Administration (NOAA). 1981. Climates of the States. 2 Vols. Detroit: Gale Research Company.

Norusis, Marija J. 1986. The SPSS Guide to Data Analysis. Chicago: SPSS, Inc.

Nuttonson, M. Y. 1965. Global Agroclimatic Analogues for the Northern Great Plains Region of the United States and an Outline of its Physiography, climate, and Farm Crops. Washington DC: American Institute of Crop Ecology.

Organization for Economic Cooperation and Development (OECD). 1979. Apriculture in the Planning and Management of Peri-Urban Areas, Vol. 1. Paris: OECD.

O'Rourke, A. Desmond. 1978. The Changing Dimension of U.S. Agricultural Policy. Englewood Cliffs, NJ: Prentice-Hall.

Otte, Robert C. 1974. Farming in the City's Shadow: Urbanization of Land and Changes in Farm Qutput in SMSA's. 1960-1970. U.S. Dept. of Agr. publication ERS-250. Washington DC: GPO.

Paarlberg, Donald. 1970. The Forces Modemizing Farming in Corporate and Family Farming. Ames: Iowa State U. 
Palen, J. J. 1975. The Urban World. New York: McGraw-Hill.

Paludan, C. T. N. 1968. "The Use of Mathematical Models to Describe Agricultural Land Use." In Geography Symposium Topics. Denver: U. of Denver.

Papadakis, Juan. 1952. Agricultural Geography of the World. Buenos Aires.

Pedhazur, E.J. 1982. Multiple Regression In Behavioral Research. New York: Hold, Rinehart.

Peet, J. Richard. 1967. "The Present Pertinence of Von Thünen Theory." Annals of the Association of American Geographers, 57:810-811.

Peet, J. Richard. 1969. "The Spatial Expansion of Commercial Agriculture in the Nineteenth Century: A Von Thunen Interpretation." Economic Geography, 45:283301.

Peterson, A. 1944. Thunen's Isolierte Staat: Die Landwirschaft als Glied der volkswirtscaft. An English edition of Von Thunen's original. In Hall (Ed.), Von Thunen's Isolated State, London: Pergamon Press.

Philbrick, Allen K. 1967. This Human World. New York: John Wiley.

Phipps, M., and D. Julian. 1983. "Application of Information Theory to the Study of Agricultural Land Use Adjustment to the Natural Environment." In John W. Frazier et al (eds.), Papers and Proceedings of Applied Geography Conferences, Vol. 6.

Plant, Thomas R. 1980. "Urban Expansion and the Loss of Farm Land in the United States: Implications for the Future." American Joumal of Agricultural Economics, 62:532-542.

Predöhl, A. 1928. "The Theory of Location and its Relation to General Economics." Ioumal of Political Economy, 36:371-390.

Preston, James E. 1952. "Toward A Further Understanding of the Regional Concept." Annals of the Association of American Geographers, 42:195-222.

Rand McNally Commercial Atlas and Marketing Guide. 1986. 117th Edition. Chicago: Rand McNally.

Redman, Barbara J. 1980. "Household Expenditures on Meat Versus Nonmeat Sources of Protein in the United States." Southem Joumal of Agricultural Economics, 12(2):5154.

Reimund, D. A. 1975. Earming and Agribusiness Activities of Large Multiunit Firms. U.S. Dept. of Agr. publication ERS-591. Washington DC: GPO.

Renuad, Bertrand M. 1972. "On a New Classical Model of Land Use." The Swedish Journal of Economics, 74(3):400-404.

Reynolds, Lloyd G. 1979. Microeconomics. Homewood, IL: Richard Irwin, Inc. 
Ricardo, D. 1817. The Princioles of Political Economy and Taxation. Everyman's edition (1911). London: J. M. Dent \& Sons.

Richter, Hans. 1975. "Changes in Land Development Within the Peripheral Zone: Case Study of the Halle-Leipzig Agglomeration." Gegraphica Polonica, 30:95-103.

Robinson, H. 1966. Geography for Business Studies. London: Macdonald \& Evans.

Rosenberg, N. J. 1981. "Technologies and Strategies in Weather-Proofing Crop Production." In Climate's Impact on Food Supplies, Slater and Levin (Eds.). American Association for the Advancement of Sciences Symposium. Boulder, CO: Westview Press.

Ruap, P. M. 1980. "Competition for Land and the Future of American Agriculture." In The Future of American Agriculnure as a Strategic Resource, Batie and Robert (Eds.). Washington DC: Conservation Foundation.

Ruffner, J. A., and Frank E. Bair. 1987. The Weather Almanac. Detroit: Gale Research Company.

Russworm, L. H. 1967. "Expanding Urbanization and Selected Agricultural Elements: Case Study, Soutwestern Ontario Area, 1941-1961." Land Economics, 43:101-108.

Ruthenberg, Hans, and Hans E. Jahnke, Eds. 1985. Innovation Policy for Small Farmers in the Tropics: The Economics of Technical Innovation for Agricultural Development. Oxford: Clarendon Press.

Rutherford, J., M. I. Logan, and G. J. Misen. 1969. New Viewpoints in Economic Geography. Sydney, Australia: Martindale Press.

Sauer, Carl O. 1921. "The Problem of Land Classification." Annals of the Association of American Gengraphers, 11:3-16.

Schlebecker, J. T. 1973. The Use of the Land: Essays on the History of American Agriculture. Washington DC: Coronado Press.

Schmitz, Andrew, and D. Lee Bawden. 1973. The World Wheat Economy: An Empirical Analysis. Giannini Fouñdation Monograph No. 32. Berkeley: U. of California Press.

Schneider, K. 1986. "Plant Genetics Revolutionizes Production of Food." The Oregonian, (Sept. 18), p. F2.

Schultz, T. W. 1951. "The Declining Economic Importance of Agricultural Land." Economic Joumal, 61:725-741.

Schultz, T. W. 1953. The Economic Organization of Agriculture. New York: McGrawHill.

Schultz, T. W. (Ed.). 1978. The Distortion of Agricultural Incentives. Bloomington: Indiana U. Press. 
Schoenemann, j. A. 1987. "Smaller Crop Promises Better Prices for Potato Growers." American Vegetable Grower. Westem Edition, 35(1):22-25.

"Science is Not to Blame for Surpluses, Researcher Says." 1987. The Good Fruit Grower, 38(1):16.

Scott, Peter. 1957. "The Agricultural Regions of Tasmania: A Statistical Definition." Economic Geography, 33:109-121.

Sheets, K. R., et al. 1986. "A Bumper Crop of Troubles." U.S. News \& World Report, 101(7)(Aug. 18):14-15.

Show, R. H. 1980. "Climate Change and the Future of American Agriculture." In The Future of American Agriculture as a Strategic Resource, Batie and Robert (Eds.). Washington DC: Conservation Foundation.

Silk, Leonard S. 1975. Contemporary Economics. New York: McGraw-Hill.

Simmonds, N. W. 1983. "General Features of Plant Breeding." In Tsune Kosuge, et al, Genetic Engineering of Plants: An Agricultural Perspective. New York: Plenum Press.

Sinclair, Robert. 1967. "Von Thünen and Urban Sprawl." Annals of the Association of American Geographers. 57:72-87.

Sinclair, Robert. 1967. "Comment in Reply." Annals of the Association of American Geographers, 57:812-815.

Sinha, M. M. 1979. The Impact of Urbanization on Land Use in the Rural-Urban Fringe. New Delhi: Concept Publishing Co.

Slater, Lloyd E. and Susan K. Levin (Eds.). Climate's Impact on Food Supplies. Boulder, CO: Westview Press.

Smith, Darrell. 1986. "Check These Low-Cost Yield Boosters." Farm Journal, 110 (5):31.

Smith, David M. 1981. Industrial Location: An Economic Geographical Analysis. New York: John Wiley \& Sons.

Smith, J. Warren. 1920. "Rainfall of the Great Plains in Relation to Cultivation." Annals of the Association of American Geographers, 10:69-75.

Smith, Robert T., Edward J. Toaffe, and Leslie J. King. 1968. Readings in Economic Geography. Chicago: Rand McNally.

Spencer, J. E., and Norman R. Stewart. 1973. "The Nature of Agricultural Systems." Annals of the Association of American Geographers. 63:529-544.

Spencer, J. E., and Ronald J. Horvath. 1963. "How does an Agricultural Region Originate?" Annals of the Association of American Geographers, 53(1):74-92. 
Spoonser, B. and R. Netting. 1972. "Humanized Economics." Peasant Studies Newsletter, 1:54-58.

Stafford, H. A. 1967. "Factors in the Location of the Paperboard Container Industry." In Readings in Economic Geography, G. Roepke (Ed.), New York: John Wiley \& Sons.

Stringer, E. and J. S. Davies. 1966. Geography of Resources. London: Cassell and Co.

Symons, Leslie. 1967. Agricultural Geggraphy. New York: Frederick A. Praeger.

Tarrant, J. R. 1974. Agricultural Geography. New York: John Wiley and Sons.

Taylor, G. 1942. "Environment, Village and City: A Genetic Approach to Urban Geography, With Some Reference to Possibilism." Annals of the Association of American Geographers, 32:1-67.

Taylor, James A. (Ed.). 1974. Climatic Resources and Ecconomic Activity. New York: John Wiley \& Sons.

Thoman, R. S., et al. 1968. The Geography of Economic Activity. New York: McGrawHill.

Thompson, Louis M. 1962. "Evaluation of Weather Factors in the Production of Wheat." Joumal of Soil and Water Conservation, 17(4):149-157.

Toboda, Jun. 1976. "Urbanization Processes and the Rural Areas in Poland." Geographica Polonica, 33:75-83.

Turner, B. L., et al. 1977. "Population Pressure and Agricultural Intensity." Annals of the Association of American Geographers, 67(3):384-396.

Ulman, Edward L., Michael F. Dacey, and Harold Brodsky. 1969. The Economic Base of American Cities. Seattle: U. of Washington Press.

U.S. Army Corps of Engineers. 1985. Maior Waterways and Ports. Washington DC: GPO.

U.S. Department of Agriculture, Crop Reporting Board. 1980. Agricultural Prices. Economics. Statistics, and Cooperative Services. Washington DC: GPO, January 31.

U.S. Department of Commerce. 1978. Marketing Opportunities in Nonstandard Metropolitan Statistical Areas. Washington DC: GPO.

U.S. Department of Commerce. 1981. Agricultural Lands Study: Final Report. Washington DC: GPO.

U.S. Department of Commerce. 1985. Local Governments in Metropolitan Areas, 1982 Census of Governments. Bureau of the Census (GC 82[5]). Washington DC: GPO. 
U.S. Department of Commerce. 1986. National Data Book and Guide to Sources. Burreau of the Census Statistical Abstract of the United States, 106th ed. Washington DC: GPO.

U.S. Department of Transportation. 1976. National Transportation: Trends and Choices Appendix. U.S. Transportation. Washington DC: GPO.

Vale, Thomas R. 1982. Plants and People: Vegetation Change in North America. A Resource publication in geography. Washington DC: Association of American Geographers.

Visher, Stephen S. 1942. "Regionalization of the United States on a Precipitation Basis." Annals of the Association of American Geographers, 32:355-371.

Visser, Sent. 1980. "Technological Change and the Spatial Structue of Agriculture." Economic Geography, 56:311-319.

Von Eckardt, Wolf. 1964. The Challenge of Megalopolis: A Graphic Presentation of the Urbanized Northeasterm Seaboaro of the United States (based on the original study of Jean Gottmann). New York: Macmillan.

Von Thünen, J. H. 1826. Der Isolierte Staat in Beziehun auf Landwirtschaft und Nationalökonomie. Stuttgart: Gustav Fisher Verlag. English translation: P. Hall, ed., C. M. Wartenberg, tr. London: Pergamon Press, 1966.

Weaver, John C. 1954. "Crop-Combination Regions in the Middle West." The Geographical Review. 44(2):175-200.

Webb, John W. 1959. "Basic Concepts in the Analysis ofSmall Urban Centers of Minnesota." Annals of the Association of American Geographers, 49(1):55-72.

Wheeler, Douglas P. 1983. "In Defense of Farmland." Joumal of Soil and Water Conservation, 38:4.

Wicks, R. J. 1982. "The Challenge of Foreign Marketing." In Food: From Farm to Table, the U.S. Department of Agriculture 1982 Yearbook of Agriculture. Washington DC: GPO.

Winsberg, Morton D. 1980. "Concentration and Specialization in United States Agriculture: 1939-1978." Economic Geography, 56:183-189.

Wohld, Mike. 1987. "Seattle Meets A First for Wheat Srowers." Washington FarmerStockman, 112(1):1a.

Woolverton, Michael. 1987. "Mexican Tomatoes." Arizona Farmer-Stockman, 66(1):15.

World Agricultural Trade. 1978. Kansas City, MO: Federal Reserve Bank of Kansas City.

Zeimetz, Kathryn A. 1979. Growing Energy: Land For Biomass Farms. U.S. Department of Agriculture publication ERS-425. Washington DC: GPO. 